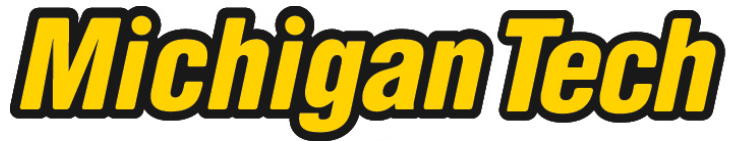 \\ Michigan Technological University Create the Future Digital Commons @ Michigan Tech
}

\section{Time-domain modeling of high-frequency electromagnetic wave propagation, overhead wires, and earth}

Nils Markus Stenvig

Michigan Technological University

Follow this and additional works at: https://digitalcommons.mtu.edu/etds

Part of the Electrical and Computer Engineering Commons

Copyright 2011 Nils Markus Stenvig

\section{Recommended Citation}

Stenvig, Nils Markus, "Time-domain modeling of high-frequency electromagnetic wave propagation, overhead wires, and earth", Master's Thesis, Michigan Technological University, 2011.

https://doi.org/10.37099/mtu.dc.etds/43

Follow this and additional works at: https://digitalcommons.mtu.edu/etds

Part of the Electrical and Computer Engineering Commons 


\title{
TIME-DOMAIN MODELING OF HIGH-FREQUENCY ELECTROMAGNETIC WAVE PROPAGATION, OVERHEAD WIRES, AND EARTH
}

\author{
By \\ NILS MARKUS STENVIG

\begin{abstract}
A THESIS
Submitted in partial fulfillment of the requirements for the degree of MASTER OF SCIENCE IN ELECTRICAL ENGINEERING
\end{abstract}

MICHIGAN TECHNOLOGICAL UNIVERSITY

2011

(C) 2011 Nils Markus Stenvig 
This thesis, "Time-domain modeling of high-frequency electromagnetic wave propagation, overhead wires, and earth", is hereby approved in partial fulfillment of the requirements for the degree of MASTER OF SCIENCE in ELECTRICAL ENGINEERING.

Department of Electrical and Computer Engineering

Signatures:

Thesis Advisor

Dr. Bruce A. Mork

Department Chair

Dr. Daniel R. Fuhrmann

Date 
To those who not only hope but believe, not only believe but have patience, not only are patient but pursue, not only pursue but persist, and not only persist, but persevere.

Fill the unforgiving minute.

Get busy living, or get busy dying.

Skål. 


\section{Table of Contents}

List of Figures $\ldots \ldots \ldots \ldots \ldots \ldots \ldots \ldots$. . . . . . . . . . . . . . .

List of Tables $\ldots \ldots \ldots \ldots \ldots \ldots \ldots \ldots \ldots \ldots$. . . . . . . . . . . . . . . . .

Preface $\ldots \ldots \ldots \ldots \ldots \ldots \ldots \ldots \ldots$ ix

Acknowledgments . . . . . . . . . . . . . . . . .

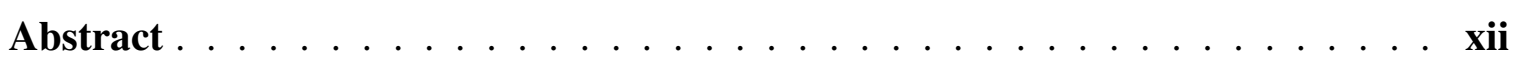

1 Introduction $\ldots \ldots \ldots \ldots \ldots \ldots \ldots \ldots$

2 Summary of Existing Work $\ldots \ldots \ldots$

2.1 HF Communication and Transmission Lines . . . . . . . . . . . . 4

2.2 Transmission Lines as Waveguides . . . . . . . . . . . . . . 5 5

2.2.1 Equivalent Circuit Based Modeling of Transmission Lines _ . . . . 5

2.2.2 Waveguides and Transmission Line Waveguide Modes . . . . . . 9

2.3 Research Methods . . . . . . . . . . . . . . . . . . . . . . . . . . . . 10

2.3.1 PLC Research Thrusts in EMC . . . . . . . . . . . . . . 11

2.3.2 State of EMC Validity for Transmission Line Performance . . . . 11

3 Modeling Issues of BPL Performance $\ldots \ldots \ldots$

3.1 Prediction of Radiated Electromagnetic Fields . . . . . . . . . . . . 15

3.1.1 Need for Realistic EMC Studies with System Components . . . . 15

3.1.2 HF Current Distribution Using EMTP-Based Line Models . . . . . 16

3.1 .3 EIGER . . . . . . . . . . . . . . . . 17

3.2 Validity of Transmission Line Models in ATP . . . . . . . . . . . 17

3.2.1 Validity for Power and PLC Frequencies . . . . . . . . . . . 20

3.2.2 Apparent Failure of Models into BPL Frequency Range . . . . . . . 23

4 HF Modeling of Transmission Lines with ATP . . . . . . . . . . . . 27

4.1 EMTP Modeling Integration with Electromagnetics-Based Models . . . . 27

4.2 Breakdown of Existing Models . . . . . . . . . . . . . . . 29

4.2.1 Review of ATP Transmission Line Theory . . . . . . . . . . . 30

4.2.2 Review of Limitations of ATP Transmission Line Model . . . . . 41

4.3 Reconciling a Closer Approximation of ATP Line Constants to EIGER . . . 44

5 Implementation $\ldots \ldots \ldots \ldots \ldots \ldots \ldots \ldots \ldots$ 
5.1 Obtaining High-Frequency Current Distribution Using ATP . . . . . . . . . 47

5.2 Predicting Radiated Fields with EIGER . . . . . . . . . . . . . . 48

5.3 Frequency-Dependent Transmission Line Implementation . . . . . . . . . . 49

5.3.1 NODA Line Constants with External Modifications . . . . . . . . . 50

5.3.2 External Vector Fitting \& Black Box Model . . . . . . . . . . . 52

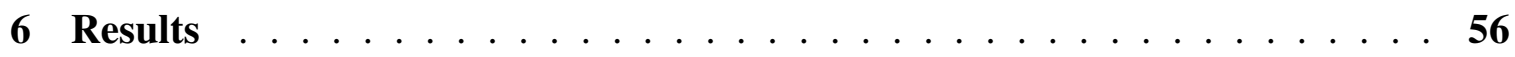

6.1 Summary of ATP-EIGER Radiation Model . . . . . . . . . . . . . . . 56

6.2 Summary of Carson vs EIGER . . . . . . . . . . . . . . . . 58

6.3 Summary of Vector Fitting . . . . . . . . . . . . . . . . . 60 60

6.3.1 Validation of the Model . . . . . . . . . . . . . . . . 61

6.3.2 Practical Example: Capacitor Bank Energization . . . . . . . 63

6.3.3 Practical Example: Lightning Impulse . . . . . . . . . . . . . . . 65

7 Conclusions and Recommendations . . . . . . . . . . . . . 67

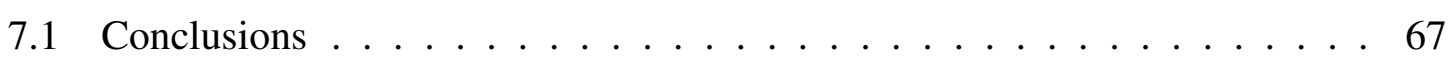

7.2 Recommendations . . . . . . . . . . . . . . . 69

7.3 Closing Comments ..................... 70

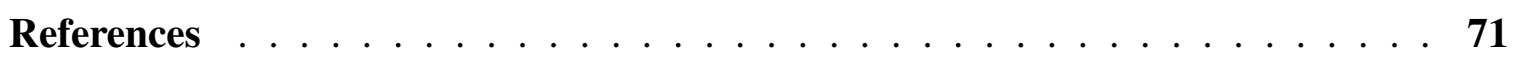

A Programming Code ......................... 75

A.1 Carson and EMTP Equations in Python, $\mathrm{C}++\ldots \ldots . . . . . .75$

A.2 EMTP Equations in Matlab . . . . . . . . . . . . . . . . . . . . 94

A.2.1 Carson's Formula . . . . . . . . . . . . . . . . . . . 94

A.2.2 Propagation Constant Calculation . . . . . . . . . . . . . 98

A.2.3 3 Conductor Carson Example . . . . . . . . . . . . 98

A.3 ATP Propagation Constants in Matlab . . . . . . . . . . . . . . . . 101

A.3.1 Reading ATP .lis File . . . . . . . . . . . . . . . . . 101

A.3.2 Calculating Propagation Constants . . . . . . . . . . . . . . 101

A.3.3 Plotting Example Code . . . . . . . . . . . . . . . 102

B Published Conference Paper . . . . . . . . . . . . . . . . . . . 104

C Documentation of IEEE Republication Permission . . . . . . . . . . . . . . 111

D Notes on Continuation of Research Work . . . . . . . . . . . . . . . . 113 


\section{List of Figures}

2.1 Telegrapher's Model . . . . . . . . . . . . . . . . . . . . . . . . . . 6

2.2 Mode Zero . . . . . . . . . . . . . . . . . . . . . . 8

2.3 Mode One . . . . . . . . . . . . . . . . . . . . . . . 8

2.4 Mode Two . . . . . . . . . . . . . . . . . . . . . 8

2.5 Foster-Equivalent for frequency-dependent $Z_{c} \ldots \ldots \ldots \ldots$. . . . . . 9

2.6 Time-domain equivalent impedance network of J. Marti . . . . . . . . . . 9

3.1 Magnitude of Carson's correction terms $\mathrm{P}$ and $\mathrm{Q}$ as a function of $h / \lambda$. . . 20

3.2 Regions of conductive and capacitive currents shown by critical frequency against earth resistivity. . . . . . . . . . . . . . . . 25

4.1 Cascaded-Pi Representation . . . . . . . . . . . . . . . . . . . . . 27

4.2 Example 4.5-km cascaded-pi line model in ATP. . . . . . . . . . . . . . 28

4.3 Attenuation constants, ATP vs EMTP Theory Book formulas for several earth resistivities . . . . . . . . . . . . . . . . . 34

4.4 Impedance magnitudes. ATP and EMTP Theory Book formulas implemented in Matlab. . . . . . . . . . . . . . . . . . . . . . . 35

4.5 Percent error of EMTP Theory Book formulas compared to ATP impedance magnitudes . . . . . . . . . . . . . . . . . 36

4.6 Attenuation constants, ATP vs Theory Book formulas for several earth resistivities . . . . . . . . . . . . . . . . . . . . 37

4.7 Impedance magnitudes. ATP and Theory Book formulas implemented in Matlab. . . . . . . . . . . . . . . . . . . . . . 38

4.8 Percent error of Theory Book formulas compared to ATP impedance magnitudes . . . . . . . . . . . . . . . . . . . . . 39

4.9 Comparison of results for Carson's series at $\theta=2 \pi / 3$ (left) and the corrected EMTP Theory Book series at the same value for $\phi$ (right) . . . . . . 39

4.10 Comparison of EMTP Theory Book, Carson, and ATP to validate derivation of impedance corrections for $\theta=\phi=0 \ldots \ldots \ldots$. . . . . . . 40

4.11 EIGER $50 \mathrm{MHz}$ vertical E-field for a $100 \mathrm{~m}$ length underneath middle of 1 $\mathrm{km}$ powerline $(500 \mathrm{~m}-600 \mathrm{~m})$. Color-legend units are $\mathrm{kV} / \mathrm{m} . ~ \ldots . . . .442$

4.12 Comparison of EIGER vertical E-fields at several frequencies for a $100 \mathrm{~m}$ length underneath middle of $1 \mathrm{~km}$ powerline $(500 \mathrm{~m}-600 \mathrm{~m}) . \ldots . . .43$

5.1 Approach to predicting radiated fields from a power system transmission line. 46

5.2 Line spacing diagram for test case scenario. Phase A and B are 1.2 and 0.3 $\mathrm{m}$ left of center. Phase $\mathrm{C}$ is $1.2 \mathrm{~m}$ right of center. . . . . . . . . . . . 47

5.3 Test case scenario with 1,000 pi-sections. . . . . . . . . . . . 47

5.4 EIGER Process . . . . . . . . . . . . . . . . . . . . . . . . 49

5.5 A black-box, multi-port, lumped-network model of a power transformer can be created from frequency-dependent nodal voltages and currents. . . . 53 
5.6 RLC circuits in ATP from circuit networks and setup for impulse measure-

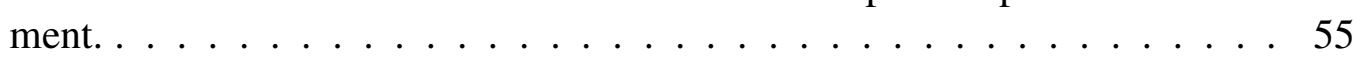

$6.1500 \mathrm{kHz}$ ATP current distribution along transmission line. . . . . . . . . . 56

6.2 Magnitude of radiated vertical magnetic field at altitude of $50 \mathrm{~m}$. . . . . 57

6.3 ATP and EIGER current distributions for fixed earth resistivity. . . . . . . . 59

6.4 ATP and EIGER current distributions for fixed frequency. . . . . . . . . . . . 60

6.5 Lab impulse testing setup with 600-kVA transformer. . . . . . . . . . . . . 61

6.6 Measured and calculated terminal voltages for ideal impulse test. . . . . . . 62

6.7 Measured and calculated terminal voltages for $R=400 \Omega$ impulse test. . . . 63

6.8 Capacitor bank energization example circuit in ATP. . . . . . . . . . . . . 64

6.9 Voltages on transformer low-winding terminals for capacitor bank energization test. . . . . . . . . . . . . . . . . . . . . . . . . . . . 64

6.10 Sending voltages and response at transformer. . . . . . . . . . . . 65

6.11 Voltages on transformer low-winding terminals for lightning impulse test. . 66 


\section{List of Tables}

$2.1 \quad$ Ratio of $h / \lambda$ for wave velocity $v=3.0 \times 10^{8} \mathrm{~m} / \mathrm{s} \ldots \ldots \ldots \ldots$

2.2 Ratio of $h / \lambda$ for wave velocity $v=\frac{1}{2} 3.0 \times 10^{8} \mathrm{~m} / \mathrm{s} \ldots \ldots \ldots \ldots$

3.1 Critical frequency values in $\mathrm{MHz}$ - depicting regions of earth behavior. $f_{\text {critical }}=10^{-6} /\left(2 \pi \varepsilon_{0} \rho\right) \mathrm{MHz}$, and assuming $\varepsilon_{0}=8.85 \times 10^{-12} \ldots \ldots 24$

3.2 Critical values of earth resistivity, $\rho(\Omega-m)$, for select frequencies. $\rho=$ $1 /\left(2 \pi \varepsilon_{0} f_{\text {critical }}\right)$, and assuming $\varepsilon_{0}=8.85 \times 10^{-12} \ldots \ldots \ldots 24$

4.1 Minimum number of required cascaded-pi line sections for accurate $1.0-\mathrm{km}$ line representation. $N=f_{\max } \times 8 l / v \ldots \ldots \ldots \ldots \ldots 28$

4.2 Coefficient $a_{i k}=4 \pi \times 10^{-4} \sqrt{5} \times D_{i k} \sqrt{f / \rho}$ for $D_{i k}=30 \mathrm{~m}$, at select frequencies and earth resistivities. . . . . . . . . . . . 32 


\section{Preface}

This thesis describes research I performed while at Michigan Tech University and Lawrence Livermore National Laboratory, from January 2009 to January 2011. As an incremental step in progress for this work, a conference paper was published through the IEEE International Symposium on Power Line Communications and its Applications (ISPLC) annual conference in March of 2010. This paper [1] is included in Appendix B and was co-authored by myself, Bruce Mork (Michigan Tech University), Barry Kirkendall (Lawrence Livermore National Laboratory), and Bob Nelson (University of WisconsinStout). In creation of the conference paper, Barry and Bob provided much of the background and results for transmission line electromagnetics, Bruce provided essential contributions in EMTP modeling theory, and I performed transmission line modeling, simulations, and data integration. The paper represents a collaborative effort of the authors, and is described in detail throughout this thesis. Any work herein that I cannot claim as my own is expressly noted as such. 


\section{Acknowledgments}

I'd like to thank my advisor, Dr. Bruce Mork for the abundance of opportunities he's given me during my time at MTU. He first gave me the opportunity to be involved with research during my senior year of undergraduate studies, which culminated in a summerlong research stint in Trondheim, Norway. When I made clear my plans to pursue my MBA, Dr. Mork convinced me also to remain in the power program as a MSEE student. Though the concurrent pursuit of graduate degrees made for a very challenging 2.5 years, they were some of the most rewarding and exciting years of my life. I thank Dr. Mork for the opportunity to be a graduate research assistant which was instrumental in my ability to afford graduate school, and was important to my academic and professional growth. I am ever grateful that through Dr. Mork's support and guidance I have traveled to Washington DC for a National Science Foundation conference, to Rio de Janeiro for an IEEE ISPLC conference, and twice to Trondheim, Norway for research. My biggest thanks for Dr. Mork, however, is for the initial encouragement (in May 2007) to become involved with undergraduate research - a decision which has had a profound impact on my life.

Over the past several years I've received funding and research support from several sources. Of course I would like to thank Michigan Tech itself, for facilitating the funding opportunities and providing an avenue through which to pursue the most leading edge research in my field. I'd like to acknowledge the National Science Foundation for their undergraduate research fellowship which afforded me the opportunity to begin research as well as to spend a summer in Norway. I'd also like to acknowledge the Lawrence Livermore National Laboratory (LLNL) of Livermore, California for their funding support and research project which resulted in the work for this thesis. Lastly, I'd like to thank the American Transmission Company for their research project and funding support during the end of my studies, and also for agreeing to hire me upon graduation. I wish the best for all these organizations, and am grateful for their support.

There were also several individuals who were instrumental in my research process. I'd like to thank Barry Kirkendall for all his help and support from LLNL. Barry was the leader for this project with BPL, and I'll always be grateful for the time I spent under his

guidance during my summer of work at the lab in Livermore. Thank you Barry for all the 
great times in Rio and our mutually favorite town of Livermore. I'd also like to thank Ted Scharlemann of LLNL, who was helpful in coding Carson's formulas, generous in allowing me to use his code, and who also made an important discovery for our research. Bob Nelson, of the University of Wisconsin - Stout, also provided a lot of help in deciphering Carson's formulas and rooting out the inherent assumptions in their derivation.

I thank my family for being the incredible supporters that they are, and for being understanding of my dedication and time commitment to school. My parents deserve all the credit for my motivation and dedication to hard work. I thank my two wonderful sisters as well for helping to keep my head on my shoulders, for always being honest with me, and for always listening when I needed to speak. I have no brothers, but I thank my friend Steve for all the fun times from pre-school onward, for always lending an ear and helping when I needed advice, and for being a great friend. I also want to thank my Grandma Leinonen, Grandma Frances, and Aunt Nancy for their unending support in everything I've ever done. I feel truly blessed to have such a wonderful family.

I thank the Houghton and Michigan Tech community for creating a safe, fun, and rewarding environment to live in. The community is a blessing to be a part of. I especially thank the women's basketball players and coaches for asking me to be a practice player. Practice every day was a way to free my mind from the stresses of school, and I thank the team for their friendships, their entertainment, and for the way they inspired, captivated, and rallied the community through one of the most memorable and prolific seasons of Michigan Tech history. Go Huskies! I also thank the many friends whose presence have come and gone, but whose impressions will last forever. I especially thank my fellow graduate friends Maria, Sarah Fay, Adam, and Jaime. Also Kyle and Zim, who helped keep my sanity during my last full semester, and Allison for helping me enjoy my last weeks in Houghton. I can't forget to mention my great friend Brett - one of the most humble, level-minded, and bald people I know. Perhaps most importantly, I would like to thank my friend $\mathrm{g}$ - whose story is inspirational, whose dedication is motivational, whose photography is sensational, and who has traveled countless miles and hours in a car with me. Thanks to all for the memories.

Thanks be to God. 


\section{Abstract}

Prediction of radiated fields from transmission lines has not previously been studied from a panoptical power system perspective. The application of BPL technologies to overhead transmission lines would benefit greatly from an ability to simulate real power system environments, not limited to the transmission lines themselves. Presently circuitbased transmission line models used by EMTP-type programs utilize Carson's formula for a waveguide parallel to an interface. This formula is not valid for calculations at high frequencies, considering effects of earth return currents.

This thesis explains the challenges of developing such improved models, explores an approach to combining circuit-based and electromagnetics modeling to predict radiated fields from transmission lines, exposes inadequacies of simulation tools, and suggests methods of extending the validity of transmission line models into very high frequency ranges. Electromagnetics programs are commonly used to study radiated fields from transmission lines. However, an approach is proposed here which is also able to incorporate the components of a power system through the combined use of EMTP-type models. Carson's formulas address the series impedance of electrical conductors above and parallel to the earth. These equations have been analyzed to show their inherent assumptions and what the implications are. Additionally, the lack of validity into higher frequencies has been demonstrated, showing the need to replace Carson's formulas for these types of studies.

This body of work leads to several conclusions about the relatively new study of BPL. Foremost, there is a gap in modeling capabilities which has been bridged through integration of circuit-based and electromagnetics modeling, allowing more realistic prediction of BPL performance and radiated fields. The proposed approach is limited in its scope of validity due to the formulas used by EMTP-type software. To extend the range of validity, a new set of equations must be identified and implemented in the approach. Several potential methods of implementation have been explored. Though an appropriate set of

equations has not yet been identified, further research in this area will benefit from a clear depiction of the next important steps and how they can be accomplished. 


\section{Chapter 1}

\section{Introduction}

Overhead line (OHL) modeling of high-voltage power lines has been well-developed for many years. Modeling for this purpose is often done using phasor domain analysis with studies also performed in the time domain from power frequencies up to transients of 1-2 MHz. A globally used software for such studies is the Electromagnetics Transients Program (EMTP), which has a non-commercial version, The Alternative Transients Program (ATP) 1 , that is widely used in research. Which line model is selected for an application is largely dependent on the type of study being performed, as various assumptions may be made for computational efficiency and ease of implementation. Assumptions limit the accuracy of mathematical formulations outside the scope for which they are derived. Experimental work with ATP OHL modeling has exposed potential inaccuracies of the models. An initial hypothesis has been that earth (grounding) assumptions of formulas used in ATP are not valid when dealing with higher frequencies (on the order of 10's of MHz), but that these same formulas are valid at lower frequencies.

ATP has been used to study the performance of Power Line Carriers (PLC), which is a communication system operating in the $30-450 \mathrm{kHz}$ range [2]. Analysis of power system interactions and behavior has been a continuously evolving field. Advanced simulation tools allow increasingly complex studies to be performed. However, not much progress has been made in modeling the behavior of the relatively new Broadband over Power Lines

${ }^{1}$ ATP is the royalty-free version of EMTP. ATP and EMTP are probably the most widely-used Power System Transients simulation programs in the world today. The EMTP users group website is hosted at www.emtp.org. 
(BPL) systems and their interactions with the transmission grid and outside world. BPL communication spans signal frequencies from 2-80 MHz, which are five to six orders of magnitude higher than the power system frequency. Mathematical models for overhead lines do not generally extend to these frequency ranges without violating assumptions used. Accurate simulation of high-frequency OHL behavior is a highly desired ability of ATP, and future work is dependent on such an advancement. Propagation modeling at BPL frequencies is largely unexplored territory for ATP.

Models used in the realm of radio science and electromagnetics are much more complete than those used in power systems studies. Full (complete) mathematical models can be derived for power lines (above or below ground) which are valid and accurate well beyond the frequency ranges of BPL. These models, however, are not useful in power system software packages due to programming complexity and inefficiency of simulation. Conversely, electromagnetics software is unsuited to include power system components such as transformers and power electronics devices. In essence, software used for power systems and software used for electromagnetics have separate capabilities which do not overlap enough to make either one useful for realistic BPL studies that include the entirety of power system behaviors and high-frequency interactions.

This thesis explores a method of combining traditional equivalent circuit-based power systems modeling and electromagnetics modeling in order to more realistically study BPL and to reconcile the differences in wave propagation modeling. The work is organized into 7 chapters.

Chapter 2 provides a comprehensive background of OHL modeling, OHL waveguides for communications, and research accomplishments and deficiencies. This prepares the essential details needed for Chapter 3, which introduces a novel approach to prediction of radiated fields in a power system by combining circuit-based and electromagnetics modeling techniques. This approach was introduced through the IEEE ISPLC 2010 con- 
ference, though there are distinct limitations and issues of validity due to assumptions built into the mathematical models used. The remainder of the paper is organized to deal with these issues.

In Chapter 4 the integration of circuit-based and electromagnetics models is explored in detail along with a thorough investigation of the standard transmission line formulas and limitations thereof. The work exposes an apparent need for new formulas which circumvent the assumptions built into those currently used. This is necessary in order to extend validity of the proposed method across the BPL frequency range. Chapter 5 explains implementation details for the resolutions of Chapter 4, and Chapter 6 reviews the results. Finally, Chapter 7 ties together the conclusions and future recommendations. 


\section{Chapter 2}

\section{Summary of Existing Work}

\subsection{HF Communication and Transmission Lines}

Use of overhead lines as communications channels has been studied by researchers and utility firms for several decades. Though telephone lines and cable TV networks already provide high-speed multimedia services, there are limitations of service areas for users to connect to these networks. Highly developed countries typically have these data communication services widespread and available. However, less developed countries have far less availability of cable TV or telephone networks despite having electric power service. Power line communication (PLC) is a system of using existing power line infrastructure to transmit information over its lines.

In 1997 the IEEE held its first conference associated with the use of electric distribution lines as communications channels - the International Symposium on Power Line Communications and its Applications (ISPLC). Researchers in academia, industry professionals, and regulators attend the annual ISPLC to disseminate research in areas such as channel characterization, electromagnetic compatibility (EMC), smart grids, broadband applications, and business prospectives. The results become more promising each year, and recent research has even begun to address BPL issues. Using overhead lines for BPL purposes introduces a much greater need for very accurate predictive modeling techniques, and a need for understanding of waveguides and antenna theory. 


\subsection{Transmission Lines as Waveguides}

When operated at very high frequencies, an overhead line behaves as a large, traveling wave antenna with a directional radiation pattern [3]. The electrical nature of transmission lines can typically be captured with circuit-based models, however, the use of electromagnetic models becomes necessary when the transmission line is used as a waveguide. Waveguides and modes of propagation are critical to understand in order to be able to model transmission lines as waveguiding structures.

\subsubsection{Equivalent Circuit Based Modeling of Transmission Lines}

As described in Chapter 1, the well known EMTP-type software ATP has extensive features for modeling realistic power systems. ATP simulation tools have been successfully applied by many researchers to determine PLC performance of transmission networks $\llbracket 1,4$, 5]. Development of presently used distributed-parameter transient transmission line models for these cases are based on the phasor-domain "traveling wave model" or "telegrapher's model" presented in many textbooks [6]. The representation for a single-conductor case is shown in Figure 2.1. Note that distance $x$ is measured from the receiving end toward the sending end.

For a general multiple-conductor case, the well-known equations are

$$
-\frac{\delta V}{\delta x}=[Z] I \text { and }-\frac{\delta I}{\delta x}=[Y] V
$$

where $V$ and $I$ are the vectors of node voltages and line currents at a distance $x$ from the receiving end of the multiple conductor transmission line. $Z$ is the matrix of coupled series impedances of the conductors for an incremental length, and $Y$ is the matrix of coupled shunt admittances for that same length. More details of the solution are highlighted by 


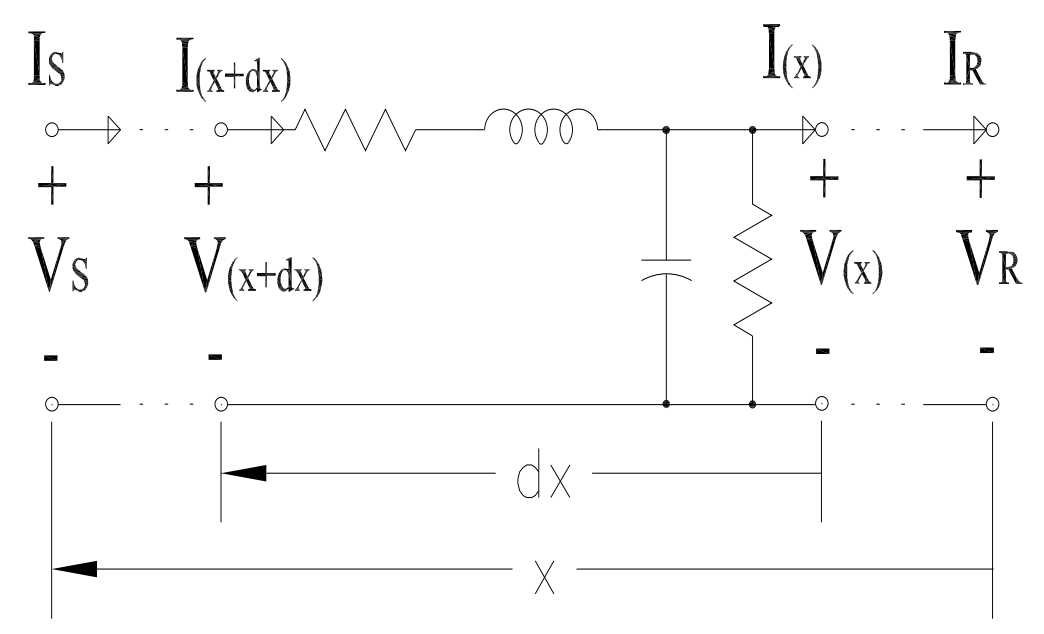

Figure 2.1: Telegrapher's Model

Greenwood [6] and several authors of IEEE publications [7, 8, 9, 10, 11]. The equations from 2.2.1 can be combined to form

$$
\frac{\delta^{2} V}{\delta x^{2}}=[Z][Y] V \text { and } \frac{\delta^{2} I}{\delta x^{2}}=[Y][Z] I,
$$

where

$$
Z_{i j}=R_{i j}+L_{i j} \frac{\delta}{\delta t} \text { and } Y_{i j}=G_{i j}+C_{i j} \frac{\delta}{\delta t} .
$$

Each diagonal element $Z_{i i}$ represents the series self impedance per unit length of the loop formed by conductor $i$ and the ground return and each off-diagonal element $Z_{i j}$ represents the series mutual impedance per unit length between conductors $i$ and $j$. The same follows for the admittance elements of $[Y]$. Three-phase lines have significant electromagnetic coupling between conductors. By means of a modal transformation, the coupled voltages and currents may be decoupled into a new set of modal voltages and currents, each of which can be treated independently in a similar manner to the single-phase line. It would be quite advantageous to diagonalize $[Z]$ and $[Y]$, however, continuous transposition must be assumed in order to completely decouple via modal transformation. A general method of modal transformation can be used to transform the phase-domain equations into a set of 
decoupled modal-domain equations which can simplify the mathematics for model implementation:

$$
V=\left[T_{v}\right] V_{m} \text { and } I=\left[T_{i}\right] I_{m}
$$

where $V_{m}$ and $I_{m}$ are modal voltages and currents, and $\left[T_{v}\right]$ and $\left[T_{i}\right]$ are the voltage and current transformation matrices which are also used to transform $Z$ and $Y$ into their decoupled modal forms $Z_{m}$ and $Y_{m}$.

$$
\begin{aligned}
& -\frac{\delta V_{m}}{\delta x}=\left[T_{v}\right]^{-1}[Z]\left[T_{i}\right] I_{m}=\left[Z_{m}\right] I_{m} \\
& -\frac{\delta I_{m}}{\delta x}=\left[T_{i}\right]^{-1}[Y]\left[T_{v}\right] V_{m}=\left[Y_{m}\right] V_{m}
\end{aligned}
$$

ATP utilizes Karrenbauer's Transformation, which is easily expanded to an arbitrary number of phases:

$$
T=\left[\begin{array}{cccc}
1 & \ldots & \ldots & 1 \\
\vdots & 1-M & \ddots & \vdots \\
\vdots & \ddots & \ddots & 1 \\
1 & \cdots & 1 & 1-M
\end{array}\right]
$$

where $M$ is the number of phases. The inverse transformation is of the form

$$
T^{-1}=\frac{1}{M}\left[\begin{array}{cccc}
1 & \cdots & \cdots & 1 \\
\vdots & -1 & 0 & 0 \\
\vdots & 0 & \ddots & 0 \\
1 & 0 & 0 & -1
\end{array}\right]
$$

The physical representation of this for a 3-phase set of conductors is given by Figures 2.2 , 2.3 , and 2.4.

Convolution methods may then be used to convert the frequency-domain solution to a time-domain equivalent that can be implemented in time-domain simulation programs 


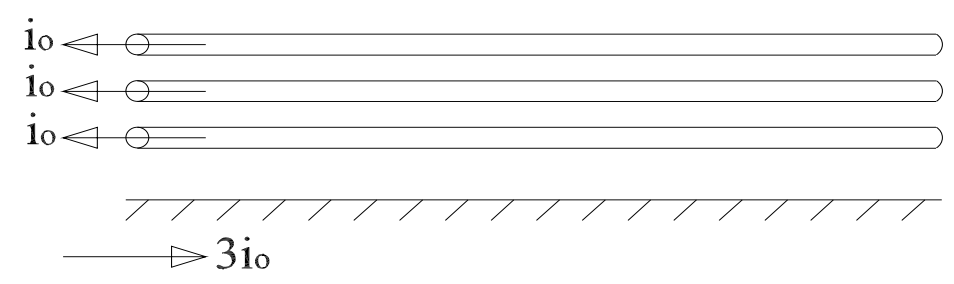

Figure 2.2: Mode Zero
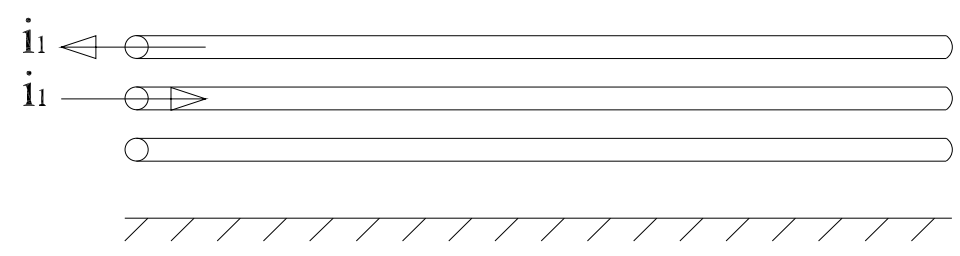

Figure 2.3: Mode One

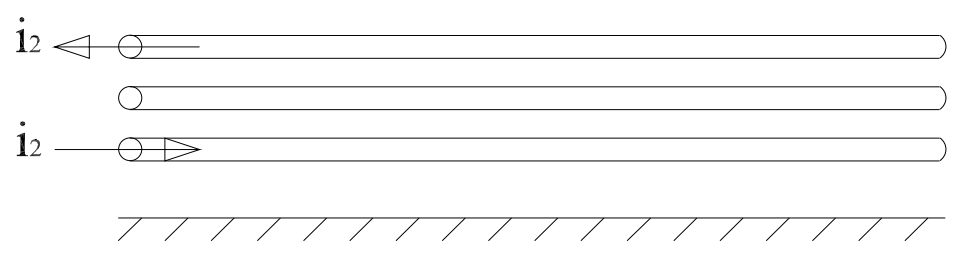

Figure 2.4: Mode Two

like EMTP. Limitations and errors in this approach are due to the fact that the solution is only valid for the frequency that the model was developed for [7, 8]. Improvements have been made by applying frequency-dependent weighting functions to the convolution [9, 10], by developing improved frequency fitting techniques [10], and by implementing the model directly in the phase domain and thus avoiding modal transformations [11]. More recent advancements include improved frequency fitting techniques [12]. In any case, it is desirable to confirm that the line model being implemented is valid within the range of frequencies to be simulated. The Foster equivalent shown in Figure 2.5 is the basis for the frequency-dependent $Z$. Figure 2.6 shows the basic representation of each end of the multi-phase Marti model [10]. Behaviors at one end manifest themselves at the other end after the appropriate propagation time delay. 


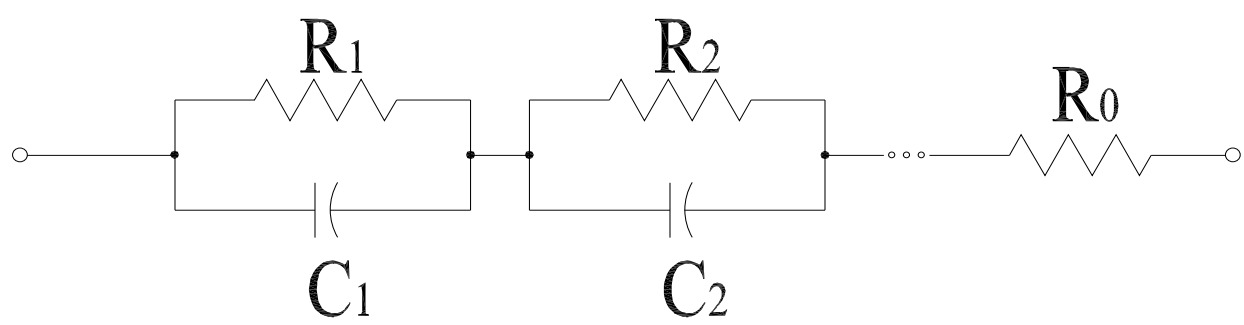

Figure 2.5: Foster-Equivalent for frequency-dependent $Z_{c}$.

\subsubsection{Waveguides and Transmission Line Waveguide Modes}

A waveguide is a physical structure designed to transmit electromagnetic energy from one point to another. Some typical waveguide structures include coaxial cables, microstrip lines, rectangular waveguides, dielectric waveguides, optical fibers, and two-wire lines. In general, there are many different electromagnetic waves that can exist independently in a waveguide. More generally, for any electromagnetic boundary-value problem, many field configurations that satisfy the wave equations, Maxwell's equations, and the boundary conditions usually exist [13]. These different field configurations (solutions) are usually referred to as "modes."

Modes in an enclosed waveguide are either propagating or evanescent. A waveguide conductor of perfect conductivity would allow propagating modes to carry energy without
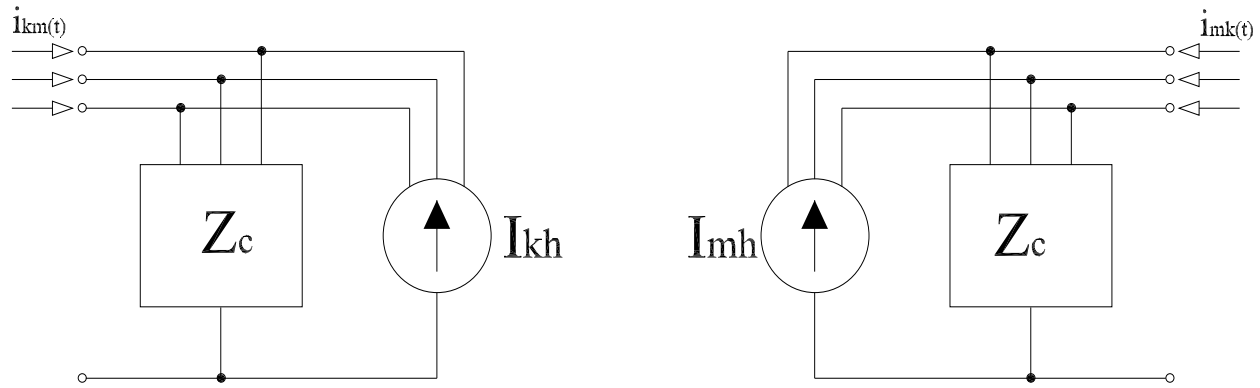

Figure 2.6: Time-domain equivalent impedance network of J. Marti 
attenuation. Evanescent modes attenuate exponentially and do not carry energy along the waveguide. A mode can switch from evanescent to propagating as the signal frequency increases to the cutoff frequency. The cutoff frequency depends on waveguide geometry and electrical characteristics. For propagating modes in realistic conductors, attenuation will exist due to the non-perfect conductivity of the waveguide.

The TEM (Transverse Electromagnetic) mode has the lowest modal cutoff frequency. This mode is "one whose field intensities, both $\mathrm{E}$ (electric) and $\mathrm{H}$ (magnetic), at every point in space are contained in a local plane, referred to as equiphase plane, that is independent of time" [13]. Simply put, the E and H fields are perpendicular (transverse) to the direction of propagation. The cutoff frequency for a TEM mode is effectively zero. The TEM mode can be present for conditions where a waveguide is formed by two or more structures that are 1) unconnected, 2) perfectly conducting, 3) parallel, and 4) in a homogenous, lossless medium. Many waveguides support what is known as a "quasi-TEM" mode (nearly a TEM mode) because the conductors and dielectrics are never perfect in reality, nor is the medium completely homogenous. Because transmission lines are typically operated at low frequencies, the TEM or quasi-TEM modes are the only significant modes of propagation.

\subsection{Research Methods}

Over the past several decades, electrical utilities have shown interest in using their already existing transmission or distribution infrastructures as a communications system. This could potentially enable these companies to compete with broadband communications companies, or at least to use the infrastructure for closed communications to operate the grid. This offers a potential solution to the "last mile" access of broadband services to isolated zones and internal networking of buildings. Several challenges confront this implementation, including noise, interference, attenuation, and transformers. In the United 
States, transformers at the distribution level typically only serve three or four customers. Transformers cause much attenuation for communications signals propagating through, making transformer bypass couplers a near necessity. Much work has been done across the globe in the area of powerline communications.

\subsubsection{PLC Research Thrusts in EMC}

The IEEE ISPLC conference was started by communications researchers in Europe and Asia as a forum for the discussion of the issues associated with the use of electrical power distribution wires as a viable communication channel. Each year, many researchers present papers regarding EMC and the use of overhead powerlines as communication channels. Works are also continuously published outside of the specific ISPLC forum. Recent publications have addressed issues with Electromagnetic Compatibility (EMC) [1, 14, 15, 16], channel modeling [17, 18, 19], and studies into higher frequency ranges [20, 21, 22]. EMC has become a popular topic due to the ever increasing trend of frequencies. Higher frequencies have a greater potential and possibility of causing electromagnetic interference (EMI) to existing radio communication systems. Governing agencies have established regulations to control the amount and ranges of interference the power system is allowed to emit. As such, the accuracy of EMI prediction becomes very important, and the inclusion of power system components in the EMC modeling causes many difficulties.

\subsubsection{State of EMC Validity for Transmission Line Performance}

To achieve relative accuracy in prediction of the performance of any natural phenomena (such as energy propagating on overhead transmission lines) one must pay attention to the limitations of the prediction model being used. As mentioned earlier, programs like EMTP are based on the "traveling wave model" or "telegrapher's model." As ob- 
served by Paul, Tesche and Olsen [23, 24, 25], one of the underlying assumptions for this model is that the electromagnetic fields surrounding the transmission line structure are TEM (transverse electromagnetic) fields - perpendicular to the direction of propagation. For the model to be strictly valid, we assume $a$ ) the conductors are parallel to each other and to the direction of propagation, $b$ ) they are perfect conductors (i.e., no resistance), and c) the conductors have uniform cross section along the line axis. In addition, $d$ ) the region surrounding the conductors is assumed homogeneous (although it can be lossy). It can also be shown (at least for two-conductor lines) that under the TEM assumption, the currents in the two conductors must be equal in magnitude and opposite in direction - i.e., that for any cross-section of the line, the total current flowing in the conductors must be zero [23]. Awareness of this set of assumptions makes it apparent that very few real life transmission lines satisfy all of these criteria.

Nearly all conductors have some resistive loss, lie over an imperfect ground (so they are immersed in an inhomogeneous material) and are not perfectly uniform in cross section. Although this is true, when examining parallel transmission lines operated at a frequency for which the cross-sectional dimensions of the line are much less than a wavelength, solution of the transmission line equations gives significant contribution to the fields and the resulting terminal voltages and currents. Such solutions are commonly referred to as "quasi-TEM" [23] or "quasi-static" [25] solutions. A vast body of research has been conducted evaluating when such solutions are accurate [26, 27, 14]. Olsen [25] points out that when the height of the transmission line is small compared to the wavelength in free space that the quasi-static approximation can be made, with the resulting solutions being identical to those derived by Carson [28]. Although these approximations may be valid at power frequencies, the situation changes when considering BPL frequencies where crosssectional dimensions of the line are no longer a fraction of a wavelength. Table 2.1 and Table 2.2 demonstrate the conditions for which this ratio becomes significant. The value of $h$ represents the height of the conductor above ground (meters). The value for $\lambda$ is calculated from $\lambda=v / f$. For both Table 2.1 and Table 2.2, the power and PLC frequencies 
(60 Hz, $30 \mathrm{kHZ}$, and $450 \mathrm{kHZ}$ ) have little risk of violating the $h / \lambda$ assumption for realistic cross-sectional dimensions. At the BPL frequencies, however, this ratio becomes a concern.

To evaluate whether or not a given model will give accurate results one must not only ask what assumptions might be violated, but also what the results will be used for. For example, in the case of a transmission line if the desired result is to determine the terminal voltages and currents to evaluate $60-\mathrm{Hz}$ power flows, quasi-static solutions obtained from solving the transmission line equations might be perfectly acceptable. If, however, one wants to determine the high-frequency electromagnetic fields radiated from the transmission lines, the error resulting from solutions based on the transmission line equations might be unacceptable. The reason is that the currents obtained from solution of the transmission line equations are truly the transmission mode (or differential line mode) currents $[23,24]$ - i.e., currents that are flowing in opposite directions. When the TEM assumptions are satisfied, these are the only currents that exist. When this is not the case, however, antenna mode (or common mode) currents can also exist [23, 24]. These are currents that are flowing in the same direction on the lines. For most power transmission line problems, the transmission line currents are dominant, so that if one wants the terminal currents and

Table 2.1

Ratio of $h / \lambda$ for wave velocity $v=3.0 \times 10^{8} \mathrm{~m} / \mathrm{s}$.

\begin{tabular}{|l|c|c|c|c|c|}
\hline $\mathrm{h}(\mathrm{m})$ & $60 \mathrm{~Hz}$ & $30 \mathrm{kHz}$ & $450 \mathrm{kHz}$ & $2 \mathrm{MHz}$ & $80 \mathrm{MHz}$ \\
\hline 10 & $2.00 \mathrm{E}-6$ & $1.00 \mathrm{E}-3$ & $1.50 \mathrm{E}-2$ & 0.0667 & $\mathbf{2 . 6 7}$ \\
\hline 20 & $4.00 \mathrm{E}-6$ & $2.00 \mathrm{E}-3$ & $3.00 \mathrm{E}-2$ & 0.133 & $\mathbf{5 . 3 4}$ \\
\hline 50 & $1.00 \mathrm{E}-5$ & $5.00 \mathrm{E}-3$ & $7.51 \mathrm{E}-2$ & $\mathbf{0 . 3 3 4}$ & $\mathbf{1 . 3 3}$ \\
\hline
\end{tabular}

Table 2.2

Ratio of $h / \lambda$ for wave velocity $v=\frac{1}{2} 3.0 \times 10^{8} \mathrm{~m} / \mathrm{s}$.

\begin{tabular}{|c|c|c|c|c|c|}
\hline h (m) $f$ & $60 \mathrm{~Hz}$ & $30 \mathrm{kHz}$ & $450 \mathrm{kHz}$ & $2 \mathrm{MHz}$ & $80 \mathrm{MHz}$ \\
\hline 10 & $4.00 \mathrm{E}-6$ & $2.00 \mathrm{E}-6$ & $3.00 \mathrm{E}-2$ & 0.133 & 5.34 \\
\hline 20 & $8.01 \mathrm{E}-6$ & $4.00 \mathrm{E}-6$ & $6.00 \mathrm{E}-2$ & 0.267 & 10.7 \\
\hline 50 & $2.00 \mathrm{E}-5$ & $1.00 \mathrm{E}-2$ & 0.150 & 0.667 & 26.7 \\
\hline
\end{tabular}


voltages, approximate results based on transmission line theory may be perfectly adequate. It turns out, however, that in the case of radiated fields antenna mode currents tend to be very significant - even if they are much smaller in magnitude than transmission line mode currents [29]. According to Paul [23] and Tesche [24] the reason is because the radiated fields from transmission line currents tend to subtract but those from antenna mode currents add.

To address the concern of interference potential from BPL signals propagating on power lines, researchers have turned to a number of strategies to predict the antenna mode currents (from which the resulting fields can be determined). One method is to use techniques commonly employed by those working with antennas and with other high-frequency applications of electromagnetics. A number of methods are available in the computational electromagnetics area, including the moment method, the finite element method, the finite difference method, and a host of others [24]. 


\section{Chapter 3}

\section{Modeling Issues of BPL Performance}

\subsection{Prediction of Radiated Electromagnetic Fields}

Prediction of the radiated electromagnetic field from any antenna involves two steps: determination of the current distribution on the antenna, followed by determination of the resulting electromagnetic fields. Carrying out these steps when the antenna is a realistic power system is a daunting task. As part of this research project, a novel two-step solution was outlined and presented as a 2010 ISPLC paper [1], also included in Appendix $\mathrm{B}$. This work introduced a unique method of applying EMTP-based transmission line models to determine the current distribution (current in each conductor and ground as a function of distance $x$ along line), which is used to determine the radiated electromagnetic fields.

\subsubsection{Need for Realistic EMC Studies with System Components}

One of the difficulties encountered when using high-frequency methods to examine the radiated fields from practical power lines lies in modeling the multitude of components in a practical power system (i.e., transmission lines, transformers, capacitor banks, etc.). Ideally, radiated fields from BPL sources could be predicted entirely from electromagnetics programs. High-frequency techniques tend to work well for things like the transmission lines themselves (since they can be modeled as wires), but get cumbersome when other 
power system components are included in the model. Programs like EMTP-ATP, however, already have lumped models for most of the power system components available. For research to progress, it would be essential to include the multitude of passive and active components of the power system in order to provide more accurate results.

\subsubsection{HF Current Distribution Using EMTP-Based Line Models}

Distributed line currents and voltages are of particular interest in simulation of line performance for communications. These values are particularly important for determining the radiated fields, which are also of interest. The robust and flexible nature of EMTPtype software (e.g. ATP) makes it an ideal platform for carrying out such work. The power system modeling features of ATP are extensive and are used across the globe for time-domain analysis. An area that has yet to be explored, however, is in high resolution modeling of distributed currents along transmission lines.

A powerful "Line \& Cable Constants" (LCC) feature of ATP is used for building transmission lines and for calculating impedance matrices [30]. For short-line modeling, the pi approximation has been widely used. For the characteristic power frequencies there is no need to obtain highly detailed current distributions along the lines. In order to study the effects of PLC at much higher frequencies, however, the decreasing wavelengths make these highly detailed models increasingly important. The resolution of current distributions must befit the frequency being used in order to accurately calculate the radiated fields (see Equation 4.1 and Table 4.1). A cascaded-pi approach within ATP is capable of meeting these needs, however, EMTP-ATP capabilities have not been validated for such high frequencies. 


\subsubsection{EIGER}

The Electromagnetics Interactions Generalized (EIGER) code was developed by the University of Houston, Sandia National Laboratory, and Lawrence Livermore National Laboratories. This three-dimensional, boundary element, frequency domain code allows the computation of electric and magnetic fields from arbitrary sources built with wires, patches, and surfaces. EIGER is freely available from Sandia National Laboratory (www.sandia.gov). Ideally, radiated fields from BPL sources could be predicted entirely from EIGER. However, as previously stated, transmission lines contain passive and active devices for power distribution control which cannot easily be built in EIGER; transformers being one example. Therefore, a new approach [1] was developed to utilize both EIGER and EMTP-type software. This novel approach for determination of radiated electromagnetic fields is continued in Chapters 4 and 5 .

\subsection{Validity of Transmission Line Models in ATP}

Carson's formulas are used in the EMTP supporting routines for Line Constants and Cable Constants, although an extension of the formula is also used in Cable Constants to account for a multi-layered stratified earth. A formula by Pollaczek is described to be more general and can be used for underground cables, but is much more difficult to program - hence, why EMTP uses Carson's formula with the additional extension for cables. The effect of a real (lossy) ground is accounted for in ATP through the use of Carson's correction equations, which were first presented in 1926 [28]. From Carson's original publications,

in [28] the impedance per unit length of an overhead wire or system of wires with ground return is derived and expressed with the form

$$
R+i X=z+i 2 \omega \ln \frac{\rho^{\prime \prime}}{a}+4 \omega \int_{0}^{\infty}\left(\sqrt{\mu^{2}+i}-\mu\right) e^{-2 h \sqrt{4 \pi \omega \lambda} \mu} d \mu
$$


where $z$ is the internal resistance of the conductor, $\rho^{\prime \prime}$ is the distance between a point $(\mathrm{x}, \mathrm{y})$ and its image, $a$ is the horizontal distance between the point $(\mathrm{x}, \mathrm{y})$ and the conductor, and $\lambda$ is the conductivity of earth. The first two terms on the right hand side of Equation 3.2 represent the series impedance of the circuit if the ground is a perfect conductor. The infinite integral is the expression which accounts for the finite conductivity of earth. Carson then shows that the circuit constants and electromagnetic field in the dielectric (earth) depend on the solution of an integral with the form

$$
J(p, q)=P+i Q=\int_{0}^{\infty}\left(\sqrt{\mu^{2}+i}-\mu\right) e^{-p \mu} \cos q \mu d \mu .
$$

Carson then shows the solution of 3.2 is

$$
P=\frac{\pi}{8}\left(1-s_{4}\right)+\frac{1}{2}\left(\ln \frac{2}{\gamma}-\ln r\right) s_{2}+\frac{\theta}{2} s_{2}^{\prime}-\frac{1}{\sqrt{2}} \sigma_{1}+\frac{1}{2} \sigma_{2}+\frac{1}{\sqrt{2}} \sigma_{3}
$$

and

$$
Q=\frac{1}{4}+\frac{1}{2}\left(\ln \frac{2}{\gamma}-\ln r\right)\left(1-s_{4}\right)-\frac{\theta}{2} s_{4}^{\prime}-\frac{\pi}{8} s_{2}+\frac{1}{\sqrt{2}} \sigma_{1}+\frac{1}{\sqrt{2}} \sigma_{3}-\frac{1}{2} \sigma_{4} .
$$

The series expansions are:

$$
\begin{aligned}
& s_{2}=\frac{1}{1 ! 2 !}\left(\frac{r}{2}\right)^{2} \cos 2 \theta-\frac{1}{3 ! 4 !}\left(\frac{r}{2}\right)^{6} \cos 6 \theta+\frac{1}{5 ! 6 !}\left(\frac{r}{2}\right)^{10} \cos 10 \theta \ldots \\
& s_{2}^{\prime}=\frac{1}{1 ! 2 !}\left(\frac{r}{2}\right)^{2} \sin 2 \theta-\frac{1}{3 ! 4 !}\left(\frac{r}{2}\right)^{6} \sin 6 \theta+\frac{1}{5 ! 6 !}\left(\frac{r}{2}\right)^{10} \sin 10 \theta \ldots \\
& s_{4}=\frac{1}{2 ! 3 !}\left(\frac{r}{2}\right)^{4} \cos 4 \theta-\frac{1}{4 ! 5 !}\left(\frac{r}{2}\right)^{8} \cos 8 \theta+\frac{1}{6 ! 7 !}\left(\frac{r}{2}\right)^{12} \cos 12 \theta \ldots \\
& s_{4}^{\prime}=\frac{1}{2 ! 3 !}\left(\frac{r}{2}\right)^{4} \sin 4 \theta-\frac{1}{4 ! 5 !}\left(\frac{r}{2}\right)^{8} \sin 8 \theta+\frac{1}{6 ! 7 !}\left(\frac{r}{2}\right)^{12} \sin 12 \theta \ldots
\end{aligned}
$$




$$
\begin{gathered}
\sigma_{1}=\frac{r \cos \theta}{3}-\frac{r^{5} \cos 5 \theta}{3^{2} 5^{2} 7}+\frac{r^{9} \cos 9 \theta}{3^{2} 5^{2} 7^{2} 9^{2} 11} \ldots \\
\sigma_{3}=\frac{r^{3} \cos 3 \theta}{3^{2} 5}-\frac{r^{7} \cos 7 \theta}{3^{2} 5^{2} 7^{2} 9}+\frac{r^{11} \cos 11 \theta}{3^{2} 5^{2} 7^{2} 9^{2} 11^{2} 13} \ldots \\
\sigma_{2}=\left(1+\frac{1}{2}-\frac{1}{4}\right) \frac{1}{1 ! 2 !}\left(\frac{r}{2}\right)^{2} \cos 2 \theta \\
-\left(1+\frac{1}{2}+\frac{1}{3}+\frac{1}{4}-\frac{1}{8}\right)^{\frac{1}{3 ! 4 !}}\left(\frac{r}{2}\right)^{6} \cos 6 \theta \\
+\left(1+\frac{1}{2}+\frac{1}{3}+\frac{1}{4}+\frac{1}{5}+\frac{1}{6}-\frac{1}{12}\right)^{\frac{1}{5 ! 6 !}}\left(\frac{r}{2}\right)^{10} \cos 10 \theta \ldots \\
\sigma_{4}=\left(1+\frac{1}{2}+\frac{1}{3}-\frac{1}{6}\right) \frac{1}{2 ! 3 !}\left(\frac{r}{2}\right)^{4} \cos 4 \theta \\
-\left(1+\frac{1}{2}+\frac{1}{3}+\frac{1}{4}+\frac{1}{5}-\frac{1}{10}\right) \frac{1}{4 ! 5 !}\left(\frac{r}{2}\right)^{8} \cos 8 \theta \\
\left(1+\frac{1}{3}+\frac{1}{4}+\frac{1}{5}+\frac{1}{6}+\frac{1}{7}-\frac{1}{14}\right)^{\frac{1}{6 ! 7 !}}\left(\frac{r}{2}\right)^{12} \cos 12 \theta \ldots \\
\left(1+\frac{1}{2}+1\right.
\end{gathered}
$$

In Figure 3.1, the magnitude of Carson's correction terms (P and Q) is shown as a function of increasing $h / \lambda$. The significance of the value of $h / \lambda$ is explained in Section 3.2.1. In general, Carson's correction terms become less valid as this ratio approaches and exceeds a value of $h / \lambda=0.3$. At the time of their derivation, Carson's equations were not intended to be applicable for all situations. Since the frequencies of operation used for PLC (30 - $450 \mathrm{kHz})$ and BPL (2 - $80 \mathrm{MHz})$ extend beyond the range for which transient analysis is commonly used, a deeper understanding of the assumptions implied in the use of Carson's equations becomes pertinent. 


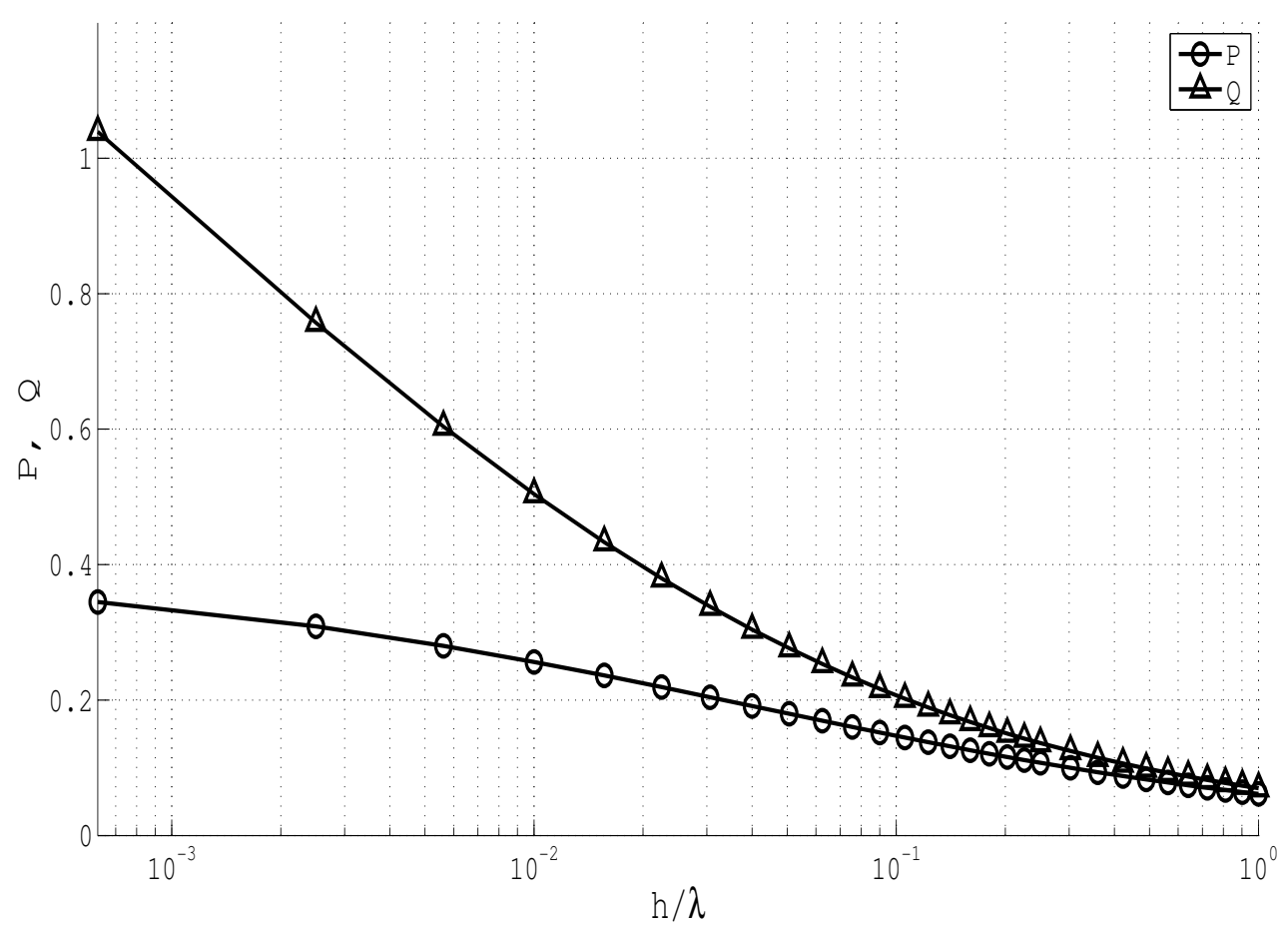

Figure 3.1: Magnitude of Carson's correction terms $P$ and $Q$ as a function of $h / \lambda$.

\subsubsection{Validity for Power and PLC Frequencies}

To understand whether the use of Carson's equations are applicable for a given situation one must have a clear understanding of what assumptions and/or approximations have been made in the derivation of the assumptions. The assumptions pertaining to Carson's

equations that are listed in Chapter 4 of the EMTP Theory Book include the following [30]:

1. The conductors are perfectly horizontal above ground, and are long enough so that the three-dimensional end effects can be neglected. Line sag is taken into account indirectly by using an average height above ground.

2. The aerial space is homogenous without loss, with permeability $\mu_{0}$ and permittivity $\varepsilon_{0}$. 
3. The earth is homogeneous with uniform resistivity $\rho$, permeability $\mu_{0}$, and permittivity $\varepsilon_{0}$, and is bounded by a flat plane with infinite extent, to which the conductors are parallel. The earth behaves as a conductor, i.e. $1 / \rho>>\omega \varepsilon_{0}$, and hence displacement currents may be neglected. Above the critical frequency $f_{\text {critical }}=1 /\left(2 \pi \varepsilon_{0} \rho\right)$, other formulas must be used.

4. The spacing between conductors is at least one order of magnitude larger than the radius of the conductors, so that proximity effects can be ignored.

Additional authors have investigated the limitations inherent in Carson's equations, and provide a more complete understanding of what assumptions and/or approximations were made in his derivations. In an invited paper written in 2000, Olsen, Young and Chang [31] reviewed the electromagnetic properties of a current on a thin horizontal wire above a flat, lossy earth. In this paper the authors outline the historical development of this problem, starting with Carson's work. The paper refers to much work of professor J.R. Wait - and highlights the contributions made by professor Wait to the solution of this problem and understanding of assumptions. The authors explicitly list several assumptions while others are implicit within the text. The assumptions which were not included in the EMTP Theory Book are summarized here:

1. The original "wire over earth" problem was of interest because of the use of systems (power transmission and telephone communications) that were operated at frequencies low enough that the wire height was a small fraction of a wavelength above earth.

2. For this case almost all of the energy from a voltage or current source is coupled into and propagates in a quasi-TEM mode. The transmission line mode is essentially the quasi-TEM mode.

3. Carson assumed that the propagation constant does not differ significantly from that 
found in the dielectric (which is typically assumed to be air) - and therefore Laplace's equation is a valid substitution for the two-dimensional wave equation in the air. This statement is equivalent to stating that Carson was focusing on the quasi-TEM mode.

4. The effect of earth conductivity on the parallel admittance per unit length is negligible.

Olsen, Young and Chang [31] then state what professor Wait showed [26] regarding Carson's assumptions. In particular, suppose $a$ is the wire radius, $j \beta$ is the propagation constant of the wave propagating on the wire, and the wave numbers in the dielectric (region 1) and ground (region 2) are $k_{1}=\omega \sqrt{\mu_{1}\left(\varepsilon_{1}-j \frac{\sigma_{1}}{\omega}\right)}$ and $k_{2}=\omega \sqrt{\mu_{2}\left(\varepsilon_{2}-j \frac{\sigma_{2}}{\omega}\right)}$, respectively. Typically region 1 is air, so $\varepsilon_{1}=\varepsilon_{0}, \mu_{1}=\mu_{0}$ and $\sigma_{1}=0$ meaning $k_{1}=\omega \sqrt{\mu_{0} \varepsilon_{0}}$. Using this notation the results of Carson are derivable from the more general case if the following conditions (described by Wait [26]) are true:

1. $\left|a \sqrt{k_{1}^{2}-\beta^{2}}\right|<<1$ This condition specifies how thin the wire must be.

2. $\left|2 h \sqrt{k_{1}^{2}-\beta^{2}}\right|<<1$ This condition specifies how high the wire must be over ground with respect to the wavelength and propagation constant.

3. $2 h>>a$ This condition specifies how high the wire must be over ground with respect to the radius of the wire.

4. $\left|k_{1} h\right|<<1$ If region 1 is air, the wavelength in free space can be expressed as $\lambda_{1}=$ $2 \pi / k_{1}$ so this condition is equivalent to $h<<\lambda / 2 \pi$ - which specifies how high the wire can be above ground with respect to the free space wavelength at the frequency of operation.

5. $\left|k_{1}^{2} / k_{2}^{2}\right|<<1$ 
Olsen et al [31] also point out that earlier results of Kikuchi [32] are embedded within Wait's work. Kikuchi [32] shows that the transmission line quasi-TEM mode used by Carson reverts to a TM mode as the frequency increases. This result emphasizes again that Carson's low-frequency quasi-TEM mode is more correctly a TM mode with a relatively small longitudinal electric field (i.e., electric field in the direction of propagation). A brief explanation is provided highlighting the fact that the quasi-TEM mode is not the only propagation mode possible for the infinitely long wire above a lossy ground. In particular, five types of waves or modes are possible - 1) spherical waves propagating into region 1; 2) spherical waves propagating into region 2;3) surface waves (or Zenneck waves) propagating along the air-ground interface; 4) the quasi-TEM mode that is actually a mode that re-directs some of the spherical wave propagation into a wave guided radiation mode that is bound to the wire, and 5) a guided Zenneck wave mode that redirects some of the energy from earth-air surface wave in the direction of the wire. The authors suggest that "the quasi-TEM modal current dominates the continuous spectrum currents over the wire if 1 ) the wire height is small relative to the free-space wavelength and 2) the earth is a reasonably good conductor at the frequencies of interest."

From the works of Olsen, Young and Chang [31] it is apparent that the effect of the quasi-TEM mode is dominant for wire heights $h<0.3 \lambda$. For $\frac{h}{\lambda}>0.3$, it appears that the spherical and surface waves begin to have a pronounced effect. From the earlier assumptions and explanations, it is clear that Carson's equations are very valid at frequencies traditionally used for power system applications. Questions regarding their validity arise as frequencies of operation extend to the BPL frequency range.

\subsubsection{Apparent Failure of Models into BPL Frequency Range}

As mentioned in the third assumption of the EMTP Theory Book [30], the accuracy of Carson's equations are subject to a critical frequency $f_{\text {critical }}=1 /\left(2 \pi \varepsilon_{0} \rho\right)$. A paper by 
Table 3.1

Critical frequency values in $\mathrm{MHz}$ - depicting regions of earth behavior. $f_{\text {critical }}=10^{-6} /\left(2 \pi \varepsilon_{0} \rho\right) M H z$, and assuming $\varepsilon_{0}=8.85 \times 10^{-12}$.

\begin{tabular}{|c|c|c|c|}
\hline$\rho(\Omega-m)$ & $0.1 f_{\text {critical }}$ & $f_{\text {critical }}$ & $2 f_{\text {critical }}$ \\
\hline 0.1 & $1.80 \mathrm{E}+04$ & $1.80 \mathrm{E}+5$ & $3.60 \mathrm{E}+5$ \\
\hline 1 & $1.80 \mathrm{E}+3$ & $1.80 \mathrm{E}+4$ & $3.60 \mathrm{E}+4$ \\
\hline 10 & $1.80 \mathrm{E}+2$ & $1.80 \mathrm{E}+3$ & $3.60 \mathrm{E}+3$ \\
\hline 100 & 18.0 & $1.80 \mathrm{E}+2$ & $3.60 \mathrm{E}+2$ \\
\hline 1000 & 1.80 & 18.0 & 36.0 \\
\hline
\end{tabular}

Semlyen, "Ground Return Parameters of Transmission Lines: An Asymptotic Analysis for Very High Frequencies" [33], provides a good explanation of this critical frequency as it applies to displacement currents, demonstrating that penetration depth approaches some finite limit at very high frequencies. Semlyen notes that $f_{\text {critical }}$ is the frequency for which the resistive current density $\left(J_{r}=E / \rho\right)$ and capacitive current density $\left(J_{c}=\varepsilon \omega E\right)$ become equal. Figure 3.2 illustrates the variation of the critical frequency as a function of the earth resistivity. For very high frequencies $\left(f>f_{\max }=2 f_{\text {critical }}\right)$ the conductive current is negligible and the earth behaves as an insulator - Region A. For lower frequencies $(f<$ $f_{\min }=0.1 f_{\text {critical }}$ ) the capacitive current is negligible and the earth behaves as a conductor - Region C. Table 3.1 and Table 3.2 help to quantify these regions of conductivity. In Table 3.1, the critical frequencies are given as a function of the earth resistivity. In Table 3.2, the equation is rearranged to show the cutoff points of earth resistivity for the frequencies associated with normal operation, PLC, and BPL.

Carson's equations do not account for capacitive currents, and are therefore only valid for Region $\mathrm{C}$. The transition region $f_{\min }>f>f_{\max }$ (Region B) is one which is diffi-

Table 3.2

Critical values of earth resistivity, $\rho(\Omega-m)$, for select frequencies. $\rho=1 /\left(2 \pi \varepsilon_{0} f_{\text {critical }}\right)$, and assuming $\varepsilon_{0}=8.85 \times 10^{-12}$.

\begin{tabular}{|c|c|c|c|c|c|}
\hline Region & $60 \mathrm{~Hz}$ & $30 \mathrm{kHz}$ & $450 \mathrm{kHz}$ & $2 \mathrm{MHz}$ & $80 \mathrm{MHz}$ \\
\hline $0.1 f_{\text {critical }}$ & $3.00 \mathrm{E}+7$ & $5.99 \mathrm{E}+4$ & $4.00 \mathrm{E}+3$ & $8.99 \mathrm{E}+2$ & 22.5 \\
\hline$f_{\text {critical }}$ & $3.00 \mathrm{E}+8$ & $5.99 \mathrm{E}+5$ & $4.00 \mathrm{E}+4$ & $8.99 \mathrm{E}+3$ & $2.25 \mathrm{E}+2$ \\
\hline $2 f_{\text {critical }}$ & $5.99 \mathrm{E}+8$ & $1.20 \mathrm{E}+6$ & $7.99 \mathrm{E}+4$ & $1.80 \mathrm{E}+4$ & $4.50 \mathrm{E}+2$ \\
\hline
\end{tabular}




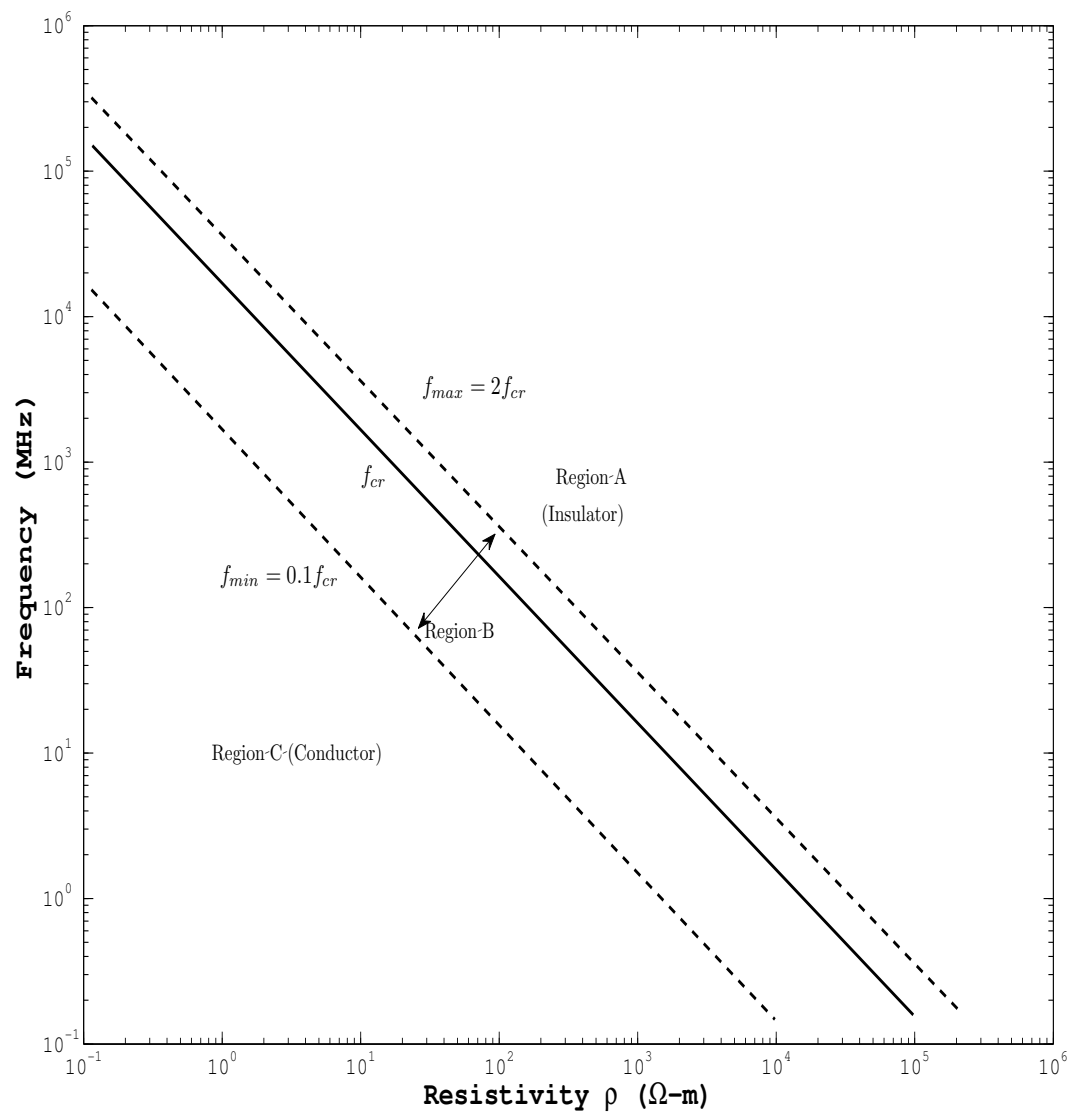

Figure 3.2: Regions of conductive and capacitive currents shown by critical frequency against earth resistivity.

cult to analyze because the earth will contain both capacitive and conductive currents which can't be ignored [33]. R. G. Olsen adds a discussion comment to Semlyen's paper, noting the importance of the decreasing wavelength in relation to conductor height above earth. Olsen suggests that a "high frequency solution to the wire above earth problem must account for continuous radiation modes and the modified Zenneck wave mode. It also must reduce to the Sommerfeld-Goubau surface wave solution [34, 35] for very high frequencies." Carson's equations for the series impedance of conductors over a lossy ground are not sufficient for all conditions at BPL frequencies. 
In attempting to predict the current distribution on overhead lines, the ratio of $h / \lambda$ (line height above ground to free-space wavelength) becomes important in verifying solution accuracy. As noted in Chapter 2, a power line is like any other waveguiding structure. As such, there are specific modes of propagation possible on the line. Carson's formulas (and all other standard transmission line formulas) are derived assuming that the dominant propagation modes are TEM (or 'quasi'-TEM, since a pure TEM mode does not exist for a wire over a lossy ground). This is indeed the dominant mode for low frequencies where the ratio of $h / \lambda$ is relatively small, however, additional modes become significant as $h / \lambda$ increases. For any waveguide, what actually happens with the currents and fields depends both on the modes that are possible and also on how the waveguide is excited. As such, even though additional modes are possible at higher frequencies, it might turn out that the currents on the lines are still dominated by the TEM modes just because of the way power lines are excited. 


\section{Chapter 4}

\section{HF Modeling of Transmission Lines with}

ATP

\subsection{EMTP Modeling Integration with Electromagnetics- Based Models}

Transmission lines have uniformly distributed parameters while pi models are lumped parameter approximations. The pi sections modeled in ATP can be used in a cascaded approach to incrementally define the line parameters. The use of cascaded-pi sections to approximate a single-phase distributed-parameter line is represented in Figure 4.1.

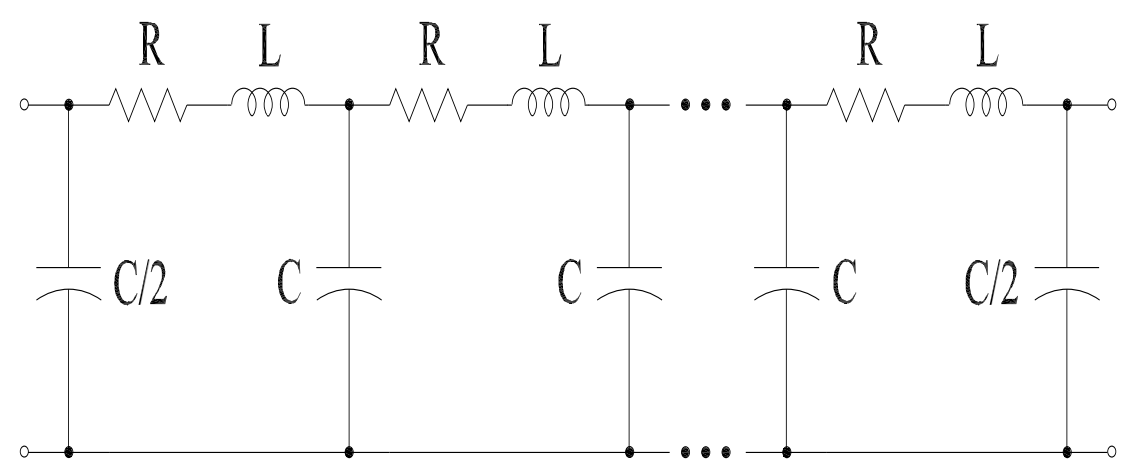

Figure 4.1: Cascaded-Pi Representation 
Table 4.1

Minimum number of required cascaded-pi line sections for accurate 1.0-km line representation. $N=f_{\max } \times 8 l / v$.

\begin{tabular}{|c|c|c|c|c|c|}
\hline$v(\mathrm{~m} / \mathrm{s})$ & $60 \mathrm{~Hz}$ & $30 \mathrm{kHz}$ & $450 \mathrm{kHz}$ & $2 \mathrm{MHz}$ & $80 \mathrm{MHz}$ \\
\hline $3.0 \mathrm{E}+8$ & $1.60 \mathrm{E}-3$ & 0.801 & 12.0 & 53.4 & $2.13 \mathrm{E}+3$ \\
\hline $1.5 \mathrm{E}+8$ & $3.20 \mathrm{E}-3$ & 1.60 & 24.0 & $1.07 \mathrm{E}+2$ & $4.27 \mathrm{E}+3$ \\
\hline
\end{tabular}

As mentioned before, it is necessary to have highly detailed line models when studying effects of higher frequencies and when dealing with increasingly small wavelengths. By shortening the line segments in the LCC modules of ATP, a finite number of short, cascaded-pi line sections can closely approximate a distributed-parameter model. By breaking down the pi model, the line currents can be obtained for each incremental pi section. The minimum number of cascaded-pi sections $(N)$ needed to accurately represent the line is determined by

$$
f_{\max }=\frac{N v}{8 l}
$$

where $f_{\max }$ is the maximum of the desired frequency range, $l$ is line length $(\mathrm{km})$, and $v$ is the propagation speed $(\mathrm{km} / \mathrm{s})$ [6]. The number of cascaded-pi sections needed is thus linked to the upper limit of the desired frequency range. As the desired frequencies become very

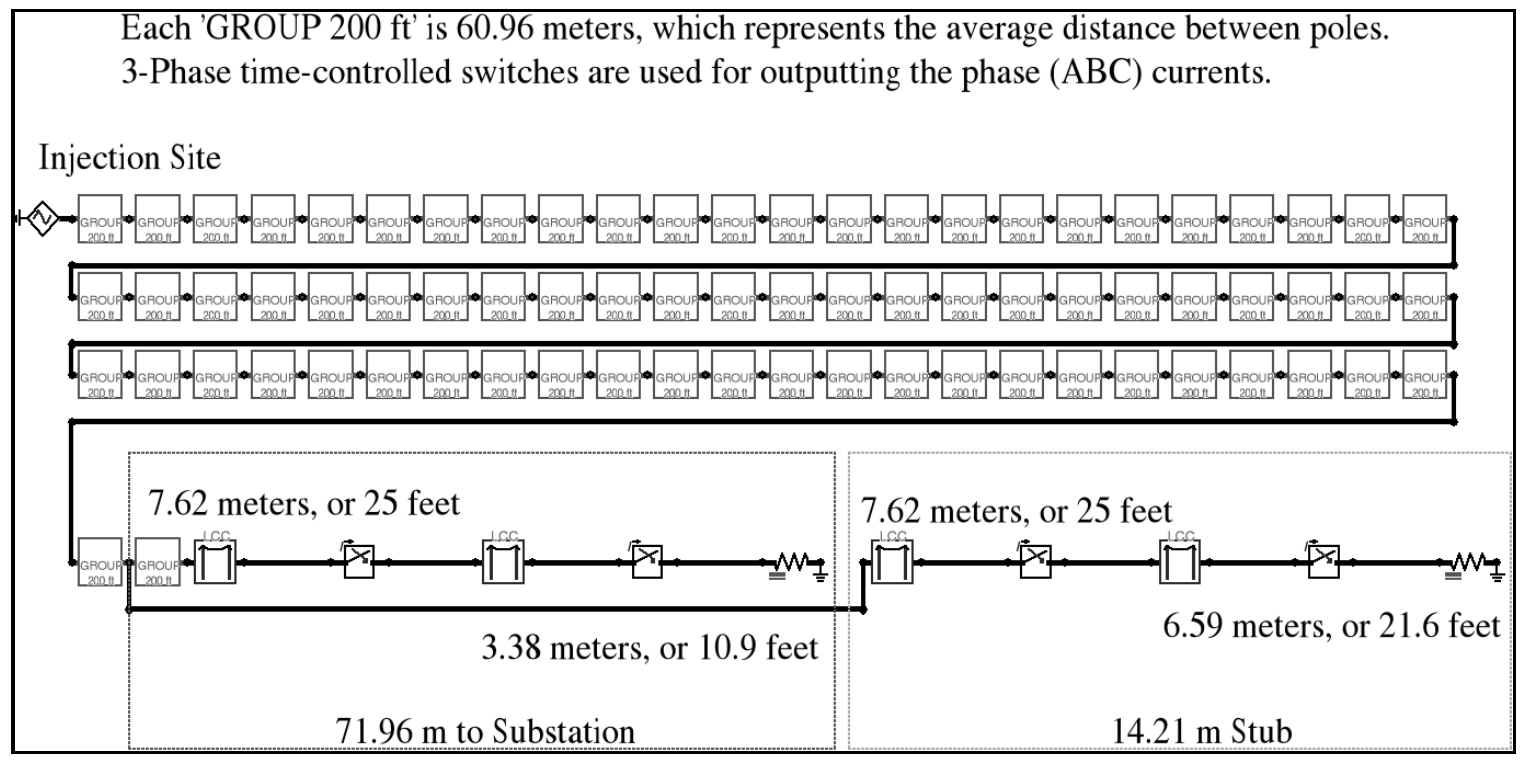

Figure 4.2: Example 4.5-km cascaded-pi line model in ATP. 
high, an obvious limitation of the cascaded approach is that a very high number of circuit elements are needed. Table 4.1 depicts this relationship for a 1-km line, showing the minimum required number of cascaded-pi sections for select frequencies and wave velocities. Since the distribution of line currents is also in question, the number of simulation outputs can also become very high. For these reasons, ATP requires a specially-compiled application file designed to accommodate the higher number of circuit elements. This version, titled 'gigmingw' is readily available through the European EMTP/ATP Users Group. Figure 4.2 displays a screenshot of a 4.5-km, 3-phase, lossless circuit in ATP. In this example, 596 pi-sections were used. According to Equation 4.1, the maximum frequency for such a model would be approximately $3.75 \mathrm{MHz}$. In the screenshot, most of the pi-sections are grouped together for aesthetics. Between each pi-section is a measuring-switch which is how the distributed currents are obtained. Because distributed line voltages and currents can be directly obtained by this method, calculation of the associated electromagnetic fields can next be achieved.

\subsection{Breakdown of Existing Models}

As mentioned in Chapter 3, the transmission line models in ATP are based heavily on the work of J. R. Carson [28]. Carson's formulas for series impedance of a conductor above ground are simplified in the EMTP-ATP reference materials [30] by Dommel. In an attempt to verify the use of these formulae within ATP, the series approximations have been implemented using Matlab. First, the formulas were implemented as described in the ATP rule book and theory book. These initial attempts did not produce good results for conditions where $a$ (see Equation 4.6) became larger than 1.0 in Carson's infinite series Equations 4.2 and 4.3. An error was found in Dommel's documentation of the equations used in EMTP/ATP, relating to the series coefficient $b$ (Equation4.7). This error was found to cause the discrepancy. Additionally, a realization was made in the way ATP handles self-inductance calculations. This resolved some issues of static offsets in self-impedance 
calculations. These issues are described in the following subsections.

\subsubsection{Review of ATP Transmission Line Theory}

The EMTP Theory Book [30], pp. 4-7 to 4-9, presents the equations used by ATP for pi-equivalent transmission line (LCC) models. These EMTP equations are based from Carson's impedance formulas introduced in Chapter 3. Note that [30] uses notations that differ from those of Carson's paper (to compare with [30], $r=a=\sqrt{p^{2}+q^{2}}$ and $\theta=\phi=$ $\left.\tan ^{-1}(q / p)\right)$. The expressions for $P$ and $Q$ in [30] are

$$
\begin{aligned}
P=\Delta R= & 4 \omega \times 10^{-4}\left\{\frac{\pi}{8}\right. \\
& -b_{1} a \cos \phi \\
& +b_{2}\left[\left(c_{2}-\ln a\right) a^{2} \cos 2 \phi+\phi a^{2} \sin 2 \phi\right] \\
& +b_{3} a^{3} \cos 3 \phi \\
& -d_{4} a^{4} \cos 4 \phi \\
& -b_{5} a^{5} \cos 5 \phi \\
& +b_{6}\left[\left(c_{6}-\ln a\right) a^{6} \cos 6 \phi+\phi a^{6} \sin 6 \phi\right] \\
& +b_{7} a^{7} \cos 7 \phi \\
& -d_{8} a^{8} \cos 8 \phi \\
& -\cdots-\}
\end{aligned}
$$


repeating in groups of four, and

$$
\begin{aligned}
Q=\Delta X= & 4 \omega \times 10^{-4}\left\{\frac{1}{2}(0.6159315-\ln a)\right. \\
& +b_{1} a \cos \phi \\
& -d_{2} a^{2} \cos 2 \phi \\
& +b_{3} a^{3} \cos 3 \phi \\
& -b_{4}\left[\left(c_{4}-\ln a\right) a^{4} \cos 4 \phi+\phi a^{4} \sin 4 \phi\right] \\
& +b_{5} a^{5} \cos \phi \\
& -d_{6} a^{6} \cos 6 \phi \\
& +b_{7} a^{7} \cos 7 \phi \\
& -b_{8}\left[\left(c_{8}-\ln a\right) a^{8} \cos 8 \phi+\phi a^{8} \sin 8 \phi\right] \\
& +\cdots-\}
\end{aligned}
$$

also repeating in groups of four. The term 0.6159315 is $1 / 2+\log (2 / \gamma)$. The correction equations used when $a \leq 5$ are Equations 4.2 and 4.3. Equations 4.4 and 4.5 are used when $a \geq 5$.

$$
\begin{aligned}
P=\Delta R & =\left(\frac{\cos \phi}{a}-\frac{\sqrt{2} \cos 2 \phi}{a^{2}}+\frac{\cos 3 \phi}{a^{3}}+\frac{3 \cos 5 \phi}{a^{5}}-\frac{45 \cos 7 \phi}{a^{7}}\right) \frac{4 \omega 10^{-4}}{\sqrt{2}} \\
Q=\Delta X & =\left(\frac{\cos \phi}{a}-\frac{\cos 3 \phi}{a^{3}}+\frac{3 \cos 5 \phi}{a^{5}}+\frac{45 \cos 7 \phi}{a^{7}}\right) \frac{4 \omega 10^{-4}}{\sqrt{2}}
\end{aligned}
$$

The equation for $a$ is shown below along with the coefficients $b, c$, and $d$ (Equations 4.7, 4.8, and 4.9) which are stored as vectors. It should be noted that the subscripts (ik) of $a$ are a matrix notation of the physical conductor geometry, while the subscripts (i) of coefficients $b, c$, and $d$ are vector notations for indexing. Table 4.2 helps in understanding how the value of $a_{i k}$ changes with frequency and earth resistivity. 


$$
\begin{aligned}
a_{i k} & =4 \pi \times 10^{-4} \sqrt{5} \times D_{i k} \sqrt{\frac{f}{\rho}} \\
b_{i} & =b_{i-2} \frac{\text { sign }}{i(i+2)} \quad \text { with the starting values }\left\{\begin{array}{l}
b_{1}=\frac{\sqrt{2}}{6} \\
b_{2}=\frac{1}{16}
\end{array}\right. \\
c_{i} & =c_{i-2}+\frac{1}{i}+\frac{1}{i+2} \text { with the starting value } c_{2}=1.3659315 \\
d_{i} & =\frac{\pi}{4} b_{i}
\end{aligned}
$$

Table 4.2

Coefficient $a_{i k}=4 \pi \times 10^{-4} \sqrt{5} \times D_{i k} \sqrt{f / \rho}$ for $D_{i k}=30 \mathrm{~m}$, at select frequencies and earth resistivities.

\begin{tabular}{|c|c|c|c|c|c|}
\hline$\rho(\Omega-m)$ & $60 \mathrm{~Hz}$ & $30 \mathrm{kHz}$ & $450 \mathrm{kHz}$ & $2 \mathrm{MHz}$ & $80 \mathrm{MHz}$ \\
\hline 0.1 & $\mathbf{2 . 0 6}$ & 46.2 & $1.79 \mathrm{E}+2$ & $3.77 \mathrm{E}+2$ & $2.38 \mathrm{E}+3$ \\
\hline 1 & $\mathbf{0 . 6 5 3}$ & 14.6 & 56.5 & $1.19 \mathrm{E}+2$ & $7.54 \mathrm{E}+2$ \\
\hline 10 & $\mathbf{0 . 2 0 6}$ & $\mathbf{4 . 6 2}$ & 17.9 & 37.7 & $2.38 \mathrm{E}+2$ \\
\hline 100 & $\mathbf{6 . 5 3 E - 2}$ & $\mathbf{1 . 4 6}$ & 5.65 & 11.9 & 75.4 \\
\hline 1000 & $\mathbf{2 . 0 6 E - 2}$ & $\mathbf{0 . 4 6 2}$ & $\mathbf{1 . 7 9}$ & $\mathbf{3 . 7 7}$ & 23.8 \\
\hline
\end{tabular}

The EMTP Theory Book [30] presents these equations as they are implemented in ATP. However, the expressions in the manual [30] differ from those presented by Carson [28]. The most significant error by Dommel can be resolved by replacing Equation 4.10 with Equation 4.11 [36]. This error is simply within the documentation, and is not present within the ATP implementation (proven later in this section).

$$
\begin{aligned}
& \operatorname{sign}=(-1)^{\left[\frac{n-1}{4} \bmod 2\right]}=1,1,-1,-1,-1,-1,1,1 \ldots \text { for } n=3,4,5,6,7,8,9,10 \ldots \\
& \operatorname{sign}=(-1)^{\left[\frac{n+1}{2} \bmod 2\right]}=1,1,-1,-1,1,1,-1,-1 \ldots \text { for } n=3,4,5,6,7,8,9,10 \ldots
\end{aligned}
$$

The sign of Equation 4.7 (coefficient $b$ ) alternates between plus and minus 1 every 2 terms. The EMTP Rule Book and Theory Book erroneously report the sign change after 
every 4 terms. This error was discovered by E. T. Scharlemann 1 [36] and has a large impact on the results (described later).

Carson's formula for the elements of the series impedance matrix $Z$ (as translated in [30]) is separated into self and mutual impedances (Equations 4.12 and 4.13 respectively).

$$
\begin{gathered}
Z_{i i}=\left(R_{i i}+\Delta R_{i i}\right)+j\left(2 \omega 10^{-4} \ln \frac{2 h_{i}}{G M R_{i}}+\Delta X_{i i}\right) \\
Z_{i k}=\Delta R_{i k}+j\left(2 \omega 10^{-4} \ln \frac{D_{i k}}{d_{i k}}+\Delta X_{i k}\right)
\end{gathered}
$$

Where $R_{i i}$ is the resistance of conductor $i, h_{i}$ is the height of conductor $i, G M R_{i}$ is the geometric mean radius of conductor $i, D_{i k}$ is the distance between conductor $i$ and the image of conductor $k$, and $d_{i k}$ is the distance between conductors $i$ and $k$.

In order to better understand the implementation of Carson's equations in ATP, the formulas from the EMTP Theory Book [30] have been reconstructed in Matlab (see Appendix A.2). Equations 4.2 through 4.13 are used to formulate the series impedance matrix $[Z]$ for a 3-conductor transmission line with configurable geometries and physical characteristics. To complete the system, the program also calculates the shunt capacitance matrix $[Y]$. The Matlab program is designed to calculate these matrices for every combination of 50 frequencies $(f)$ - $\log$ spaced between $10 \& 10^{8} \mathrm{~Hz}$ - and 50 earth resistivities $(\rho)$ - $\log$ spaced between 0.001 and $1000 \Omega-m$. A modified version of the code (see Appendix A.3) is also capable of obtaining the $[Z]$ and $[C]$ matrices from the .lis files in ATP. From the $[Z]$ and $[C]$ (or $[Y]$ ) matrices, the propagation constant $\alpha+j \beta=\sqrt{Z Y}$ can be easily calculated. In this way, a transmission line can be built in ATP and compared with the the equations from the ATP reference manual. For this initial comparison, the transmission line propagation constants $\alpha$ and $\beta$ were calculated for select combinations of $f$ and $\rho$.

${ }^{1}$ E. T. Scharlemann is a research scientist from the Global Security Directorate at Lawrence Livermore National Laboratory 


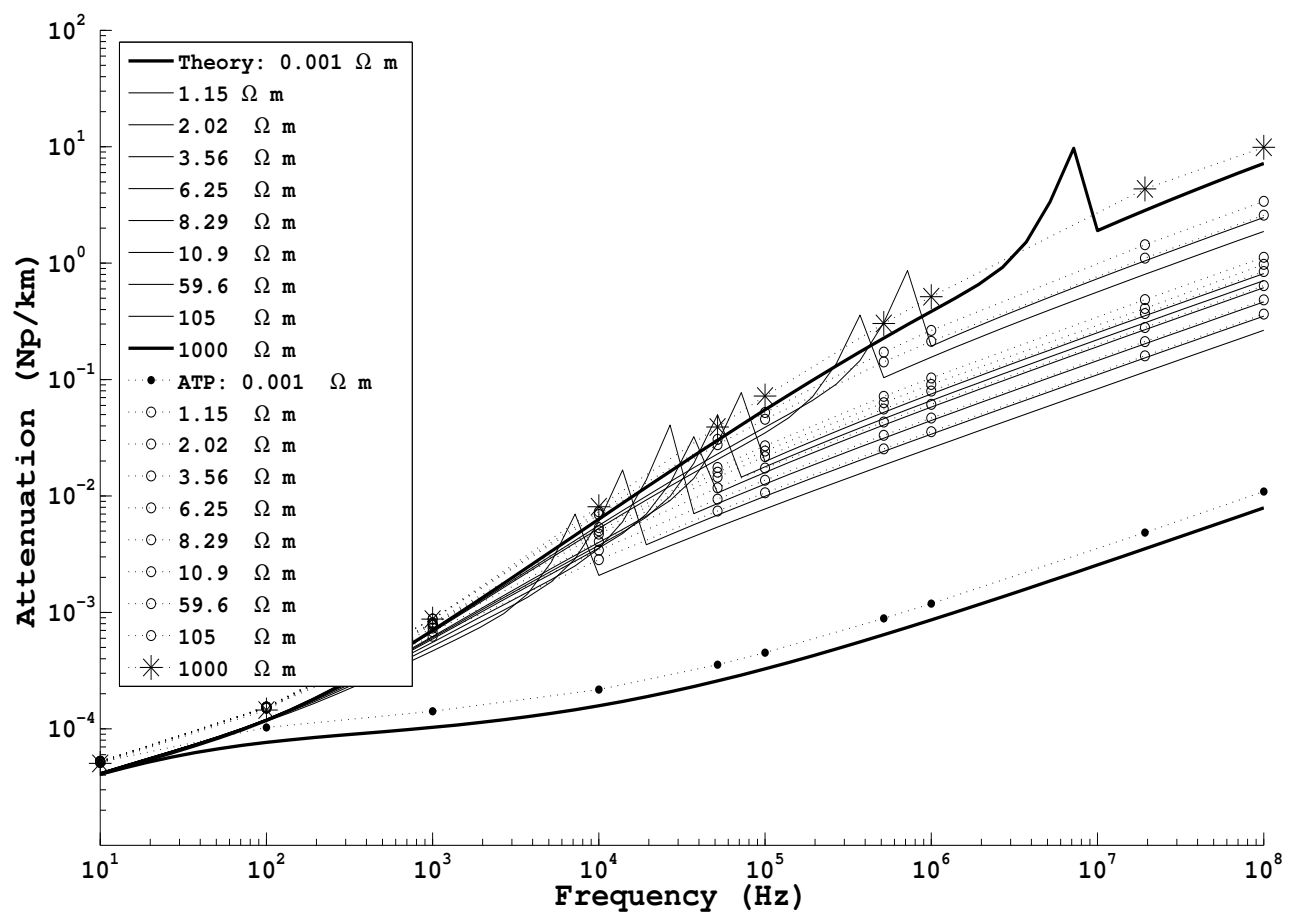

Figure 4.3: Attenuation constants, ATP vs EMTP Theory Book formulas for several earth resistivities

Plotting $\alpha$ (the attenuation constant) as in Figure 4.3, was a straightforward way of comparing theoretical results with ATP. The plot is for a three-phase system, and only the first element $\left(Z_{11}\right)$ was studied for the range of $f$ and $\rho$ previously mentioned. In Figure 4.3, the exponential spike deviation found for each value of resistivity shows where the infinite series became erroneous after $a>1.0$. Once $a \geq 5.0$ the finite series results appear fine, with the exception of the obvious static offset.

The traces in Figure 4.3 comparing the results from ATP simulation with Theory Book documentation (implemented in Matlab) were quite similar with one very major exception - the transition period between correction equations implemented from theory did not agree with ATP. For the values of frequency or $\rho$ where $a$ is between 1.0 and 5.0, the results were suspiciously deviant from the expected outcomes. For $a \geq 5.0$, Carson's finite series (see Equations 4.4 and 4.5) calculations were much better. The results from ATP 


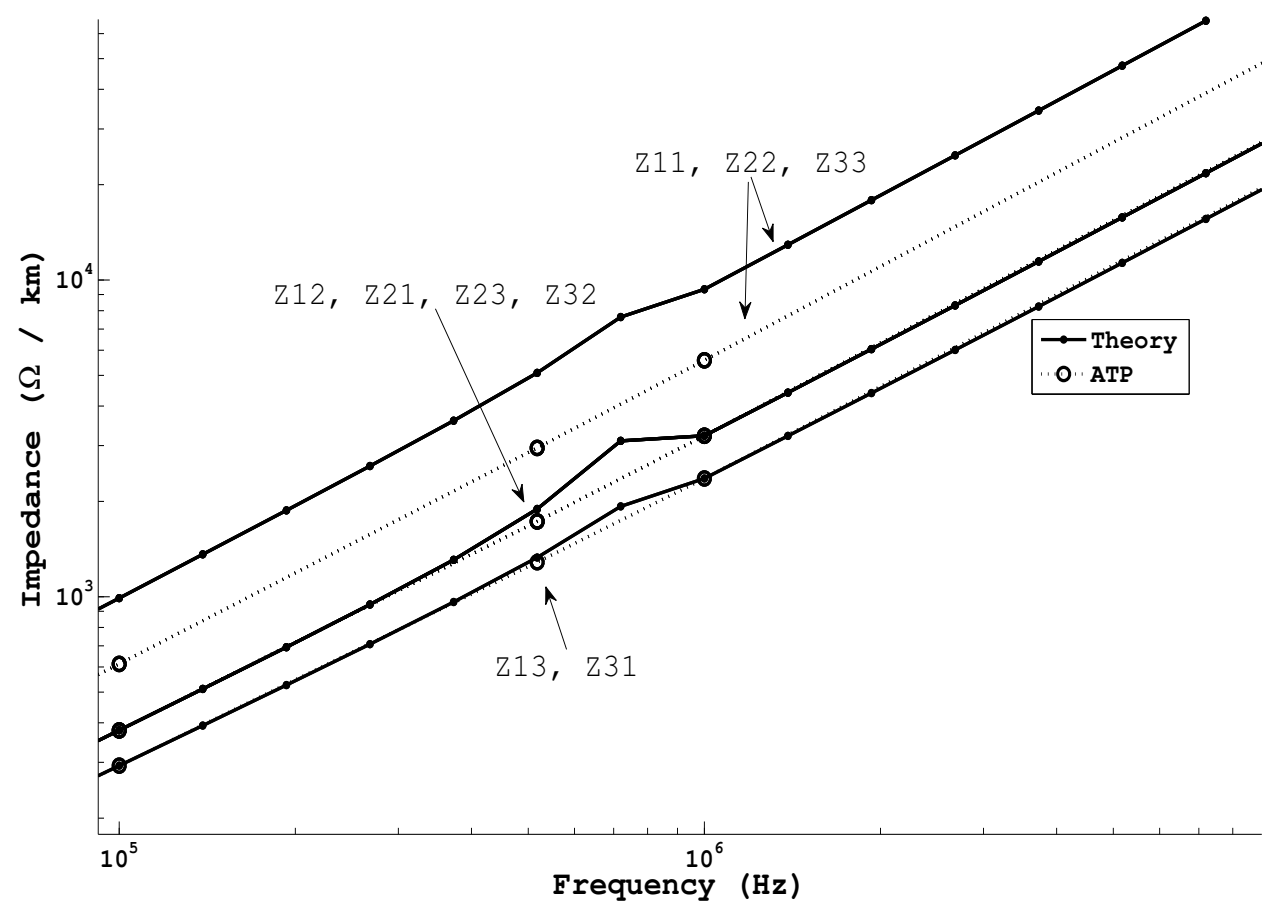

Figure 4.4: Impedance magnitudes. ATP and EMTP Theory Book formulas implemented in Matlab.

in this plot are quite good and appear to have a smooth transition between infinite and finite series calculations. This discrepancy between the ATP results and the results of the EMTP Theory Book equations is attributed to the previously mentioned error discovered by E. T. Scharlemann at Lawrence Livermore National Laboratory during the scope of this work. The corrected version of sign (Equation 4.11) is necessary to obtain accurate results that do not have the noticeable errors shown here. Regrettably, many publications refer to Dommel's EMTP Theory Book and carry the sign error in their works as well.

Figure 4.4 shows the series impedance magnitudes that resulted from the theoretical equations and from ATP. Note where the deviations occur just below $1 \mathrm{MHz}$, and the offset that occurs for the self impedance values. The offset is not an error of derivation nor implementation, but rather an issue of the way ATP calculates self inductances, described later. 


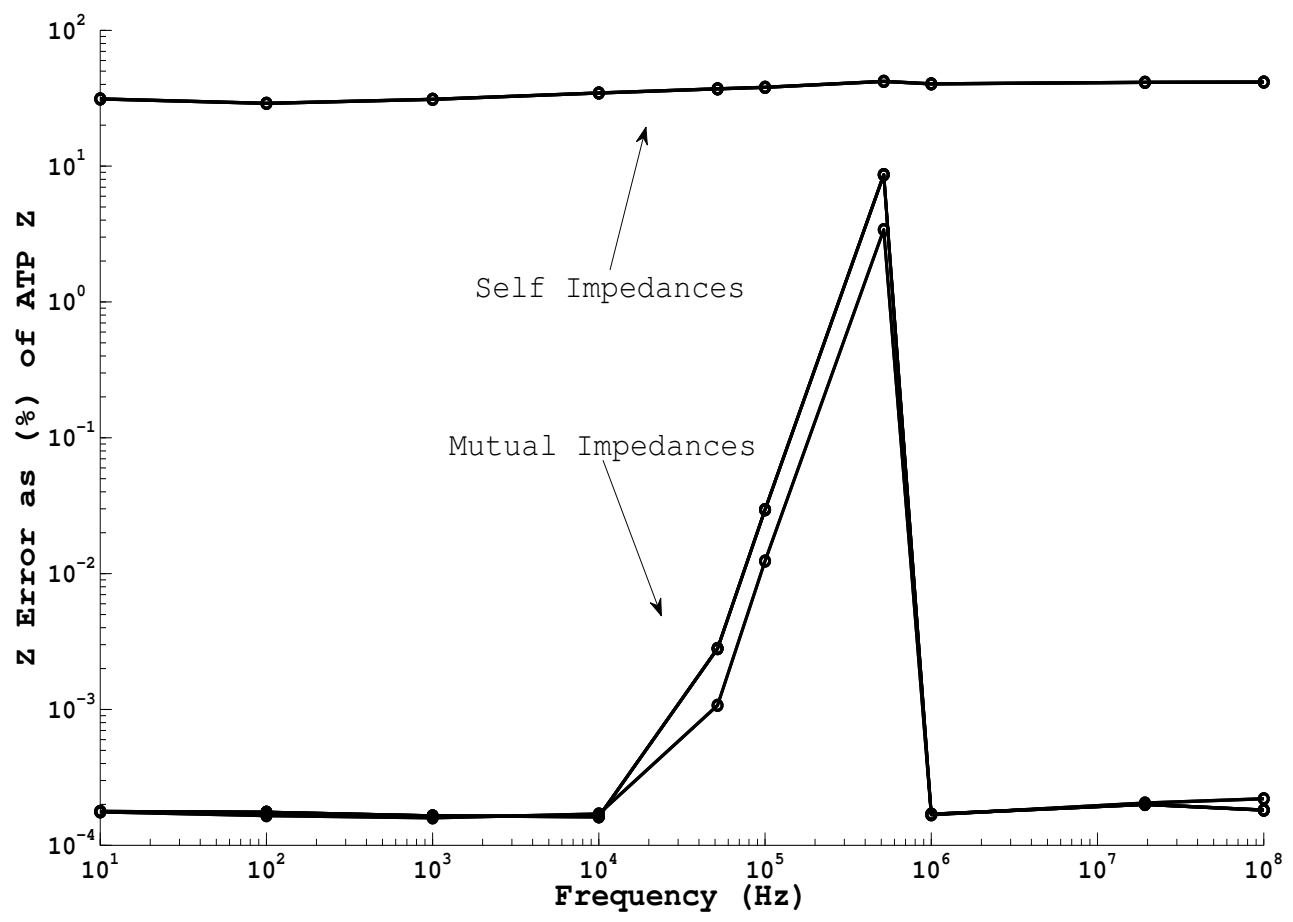

Figure 4.5: Percent error of EMTP Theory Book formulas compared to ATP impedance magnitudes

And finally, Figure 4.5 shows the percentage error between the series impedances obtained using the EMTP Theory Book and ATP (these percentages based on assumption that ATP is correct). From this plot it is apparent that the mutual impedances calculated from ATP are in very good agreement with the formulas documented in the EMTP Theory Book, while an uncharacteristic offset exists for the self impedance.

A relevant discovery from the EMTP Theory Book [30] explains the static offset in the previous plots of self impedance. In the LCC data card, there is an input called IXFlag which controls the calculation of the internal self inductance. With ATPDraw, this flag cannot be preset by the user, and the default value is 0 . The impact of this is that the reactance specified by the user in the LCC module is assumed to be correct for the user supplied frequency at one foot spacing, meaning Equation 4.12 is not the actual equation ATP uses for self impedance $Z_{i i}$. This issue is quite important to understand when trying to 
compare ATP results with Carson's equations.

When IXFlag is set to 0 the value GMR is not used at all, nor is it related to the wire radius specified by the user. Rather, a substitute for GMR is calculated which produces the user-specified reactance. This reactance, however, is an effective mutual reactance that would exist at 1-foot spacing. When ATP then calculates a self reactance, it may not be what the user expects, especially if the specified reactance is very small or zero. In the case where a user specifies the reactance to be zero, the self reactance calculated is actually: $2 \omega 10^{-4} \ln \frac{2 h_{i}}{0.3048}=5257.56 \frac{\Omega}{\mathrm{km}}$ for a $10-\mathrm{m}$ high wire and $1 \mathrm{MHz}$ frequency. Changing the wire radius will have no impact on the results.

Figures 4.6 through 4.8 are similar to Figures 4.3 through 4.5 , however, they include the effect of matching the calculation ATP makes for self inductance, as well as the

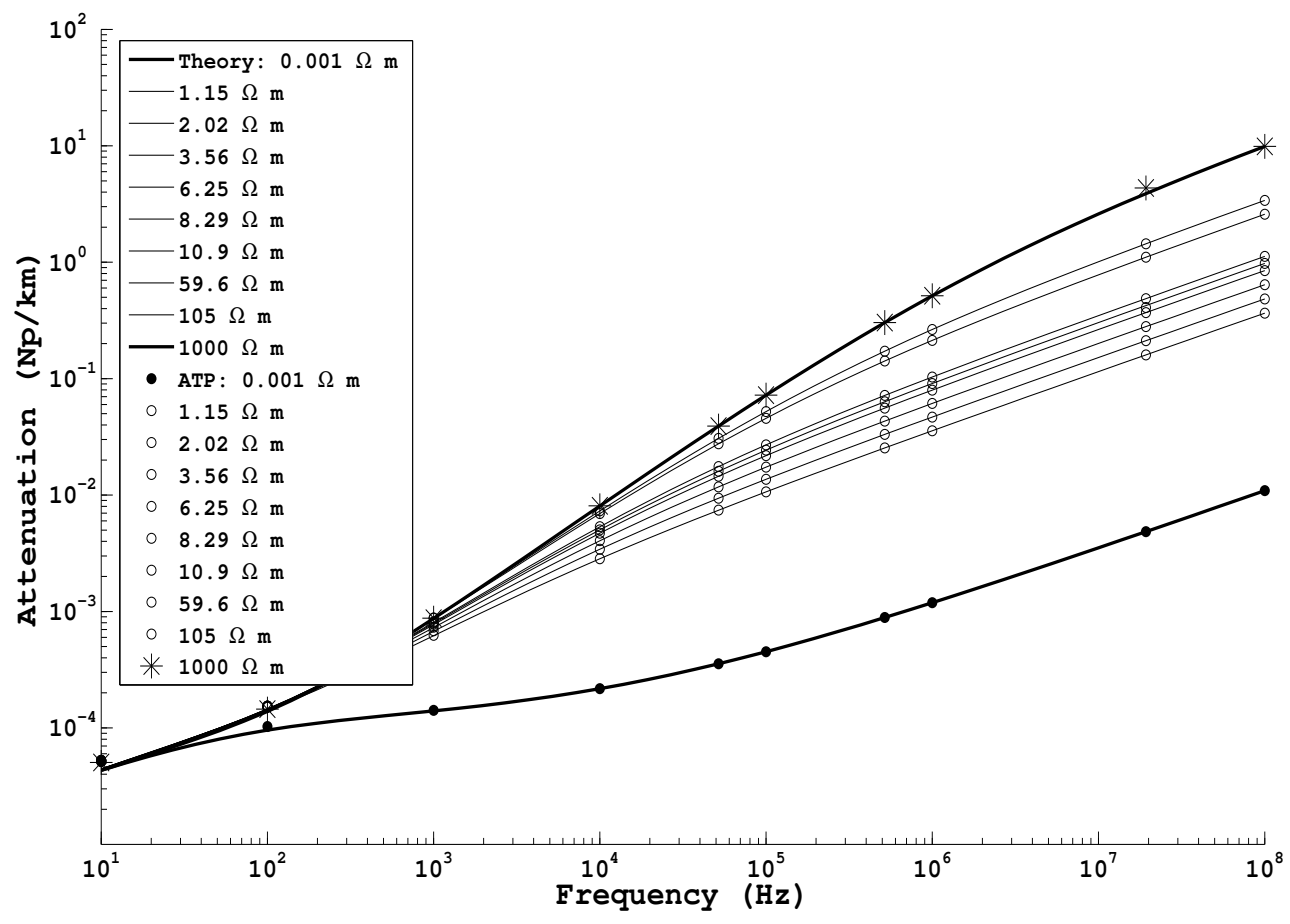

Figure 4.6: Attenuation constants, ATP vs Theory Book formulas for several earth resistivities 


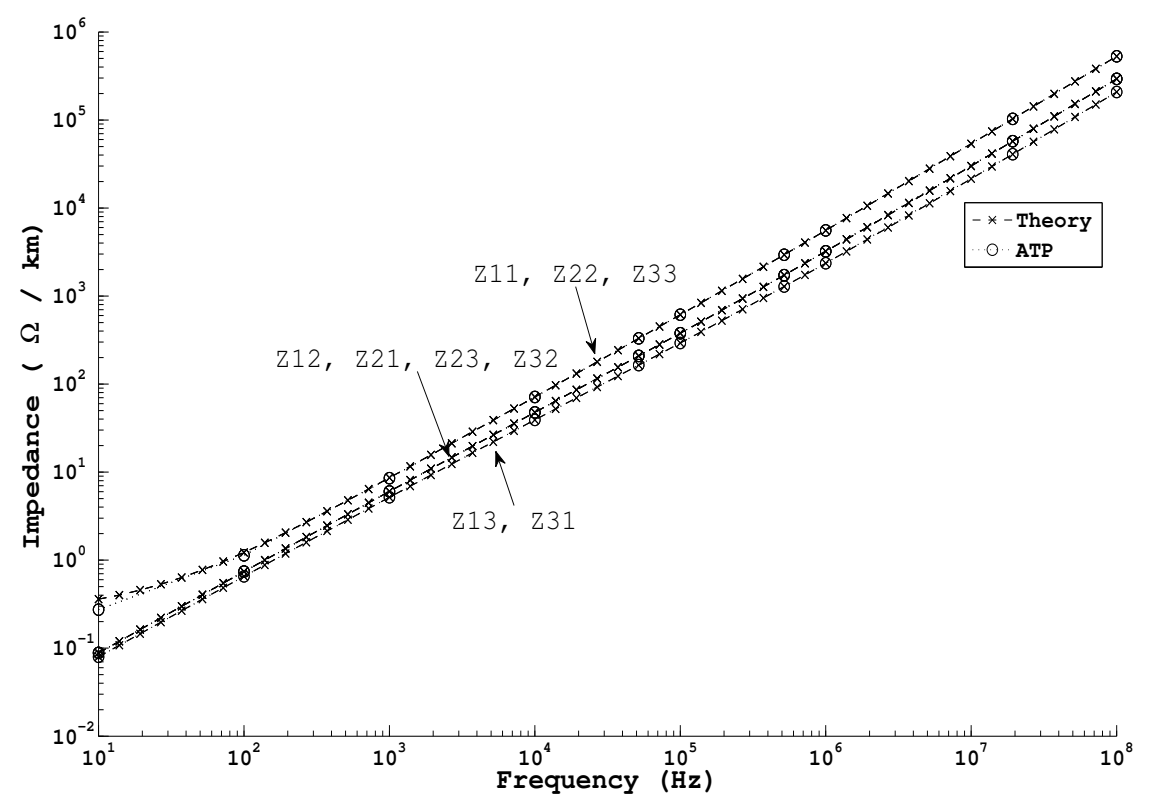

Figure 4.7: Impedance magnitudes. ATP and Theory Book formulas implemented in Matlab.

typographical correction to the infinite series coefficient $b$ which was mentioned earlier. The change in the Matlab code was simply to remove $r_{i}$ or $G M R$ from the self impedance Equation 4.12 and to replace it with $1 f t=0.3048 \mathrm{~m}$. This removes the offset in the self impedances. The change to coefficient $b$ was simply to correct a sign change that should occur every 2 terms instead of the suggested 4 terms as described in the manual [30]. This removes the discontinuity in transition between the infinite and finite series correction equations. With these corrections, all results begin to match quite reasonably. In Figure 4.8, the self impedances have a high percentage error at lower frequencies, which disappears with increasing frequency. The calculations made for the EMTP Theory Book formulas used a fixed number of correction terms for the entire range of frequencies. In ATP, the number of correction terms used varies with frequency, utilizing more correction terms as frequency increases. When fewer correction terms were used in the EMTP Theory Book calculations, the high percentage offset at lower frequencies decreased dramatically, while higher frequencies became more erroneous. This high percentage error is simply a result of using a fixed number of correction terms for all frequencies. 


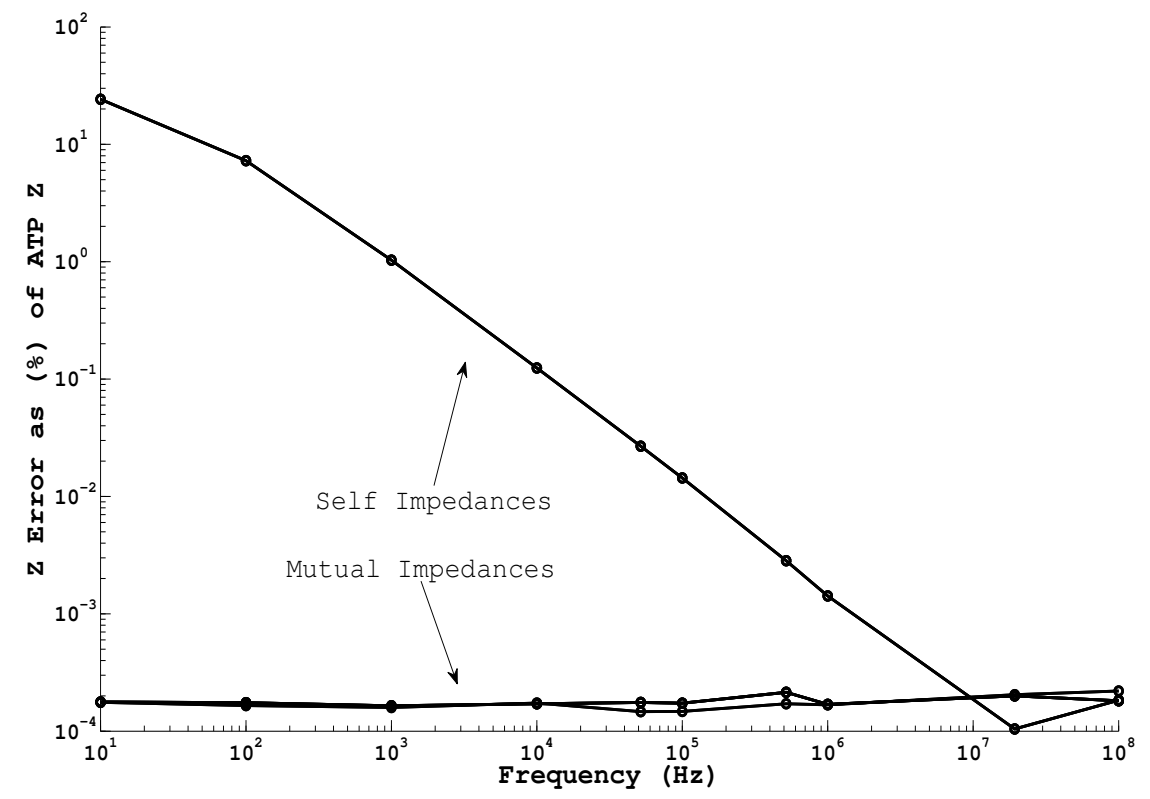

Figure 4.8: Percent error of Theory Book formulas compared to ATP impedance magnitudes

The series impedance equations from Carson's paper [28] have been replicated by E. T. Scharlemann [36] using Python programming language (see Appendix A.1) for comparison with those of the EMTP Theory Book [30]. Figure 4.9 compares the numerical results for $0<r<=10$ at $\theta=2 \pi / 3$ for the series in [28] with the corrected series from [30]. Note that for the EMTP equations, $r=a$.
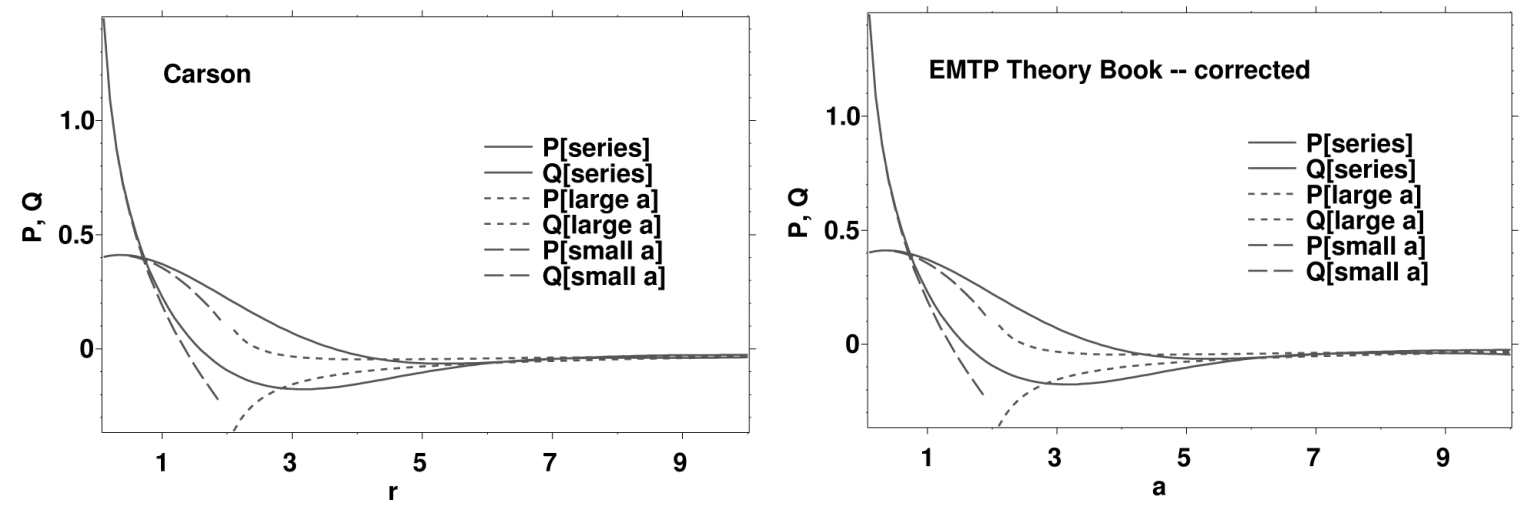

Figure 4.9: Comparison of results for Carson's series at $\theta=2 \pi / 3$ (left) and the corrected EMTP Theory Book series at the same value for $\phi$ (right). 


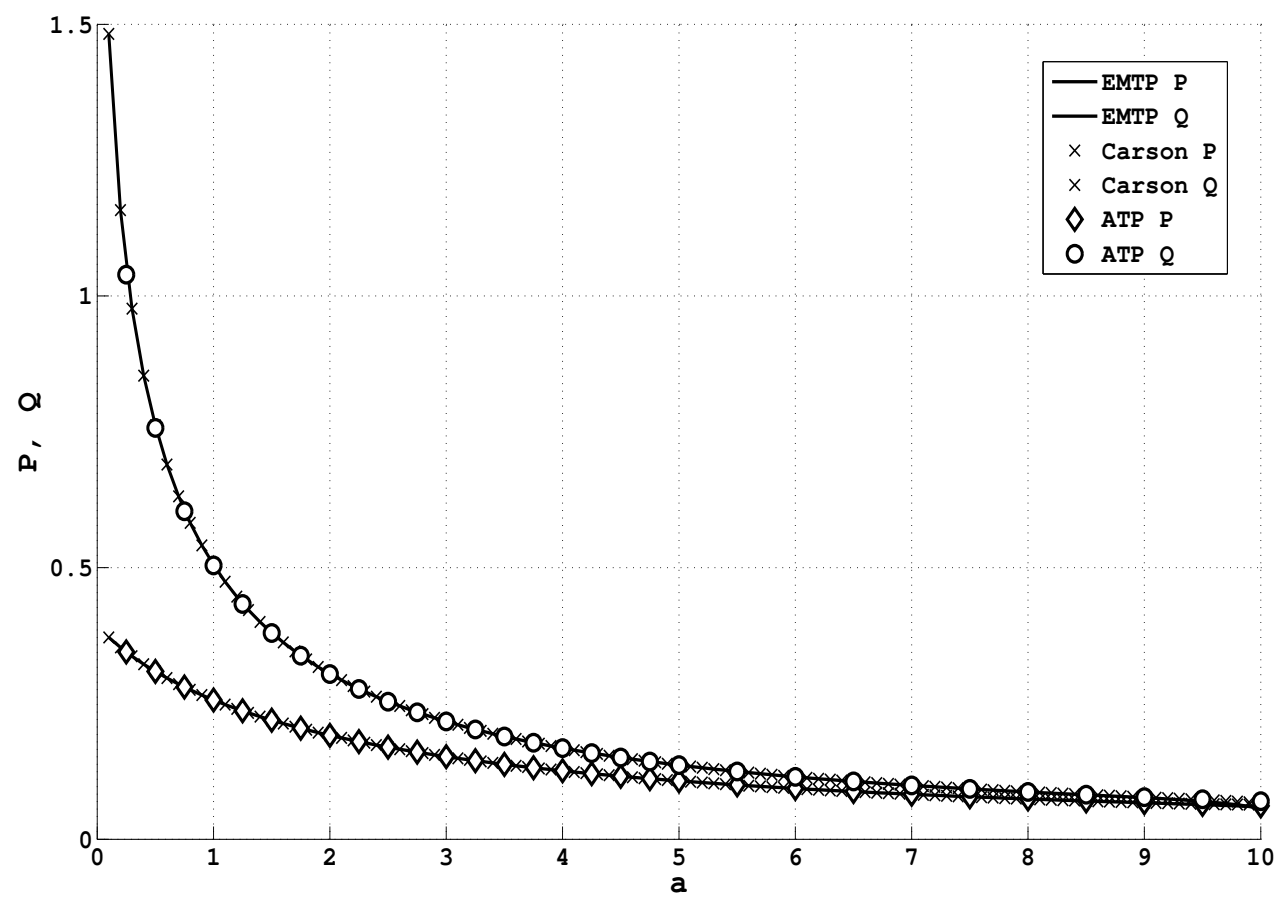

Figure 4.10: Comparison of EMTP Theory Book, Carson, and ATP to validate derivation of impedance corrections for $\theta=\phi=0$

The left axis in Figure 4.9 represents Carson's series impedance corrections due to earth return from Equations 4.2 through 4.5 . The bottom axis represents different combinations of frequency, earth resistivity and line geometry as described in Equation 4.6. From these plots it is apparent that the corrected EMTP series is commensurate with Carson's original equations. To establish evidence of congruency among ATP, Carson's formulas, and the EMTP Theory Book, the correction equation results were then compared for the series self impedances (equivalent to the case of a single conductor above ground). Figure 4.10 shows the comparison.

The description of Figure 4.10 is similar to Figure 4.9 with the exception that $P$ and $Q$ represent the self impedance corrections $\Delta R_{i i}$ and $\Delta X_{i i}$. These correction values are easy to obtain from using Carson's equations and the EMTP counterparts, however they are more difficult to retrieve from ATP. The series impedance matrices can be extracted 
from .lis files, however, the data must be processed to determine the values of $P$ and $Q$. As mentioned earlier, ATP has a nonintuitive way of calculating the self inductance when the data card IXflag is 0 . The correction impedances can be separated, however, once the process is understood. Based on this thorough investigation, ATP is in fact correctly using Carson's equations for its line \& cable constants routines, although there is a typo in the Theory Book.

\subsubsection{Review of Limitations of ATP Transmission Line Model}

In Chapters 2 \& 3 concerns were expressed regarding the usefulness and validity of Carson's equations when applied to realistic transmission line configurations and BPL frequencies. The skepticism arises based on the many assumptions described in Section 3.2.2 which suggest there are modes of propagation experienced which Carson's equations do not capture. These mode exclusions are inherent to the assumptions Carson made to derive his series expressions. Given that ATP does in fact utilize Carson's equations for its Line \& Cable Constants transmission line models, it would follow that ATP models cannot account for modes of propagation other than the quasi-TEM mode.

Utilizing the EIGER program to construct a simple transmission line, the introduction of new modes of propagation should be evident at very high frequencies. A simple 1 $\mathrm{km}$ line with a height of $10 \mathrm{~m}$ was used to predict the radiated fields for a $100 \mathrm{~m}$ section of the middle of the line 2 . Figure 4.11 shows the vertical electric field strength for a $50-\mathrm{MHz}$ line signal. Figure 4.12 shows the same information for frequencies of $100 \mathrm{kHz}, 1 \mathrm{MHz}$, $10 \mathrm{MHz}$, and $50 \mathrm{MHz}$.

The radiated fields of Figures 4.11 and 4.12 were calculated using EIGER, which also provides the distributed currents along the line. To compare EIGER and ATP for this

\footnotetext{
${ }^{2}$ All EIGER results for this work were obtained with the help of Barry Kirkendall - a research scientist with Physical \& Life Sciences Directorate of Lawrence Livermore National Laboratory.
} 


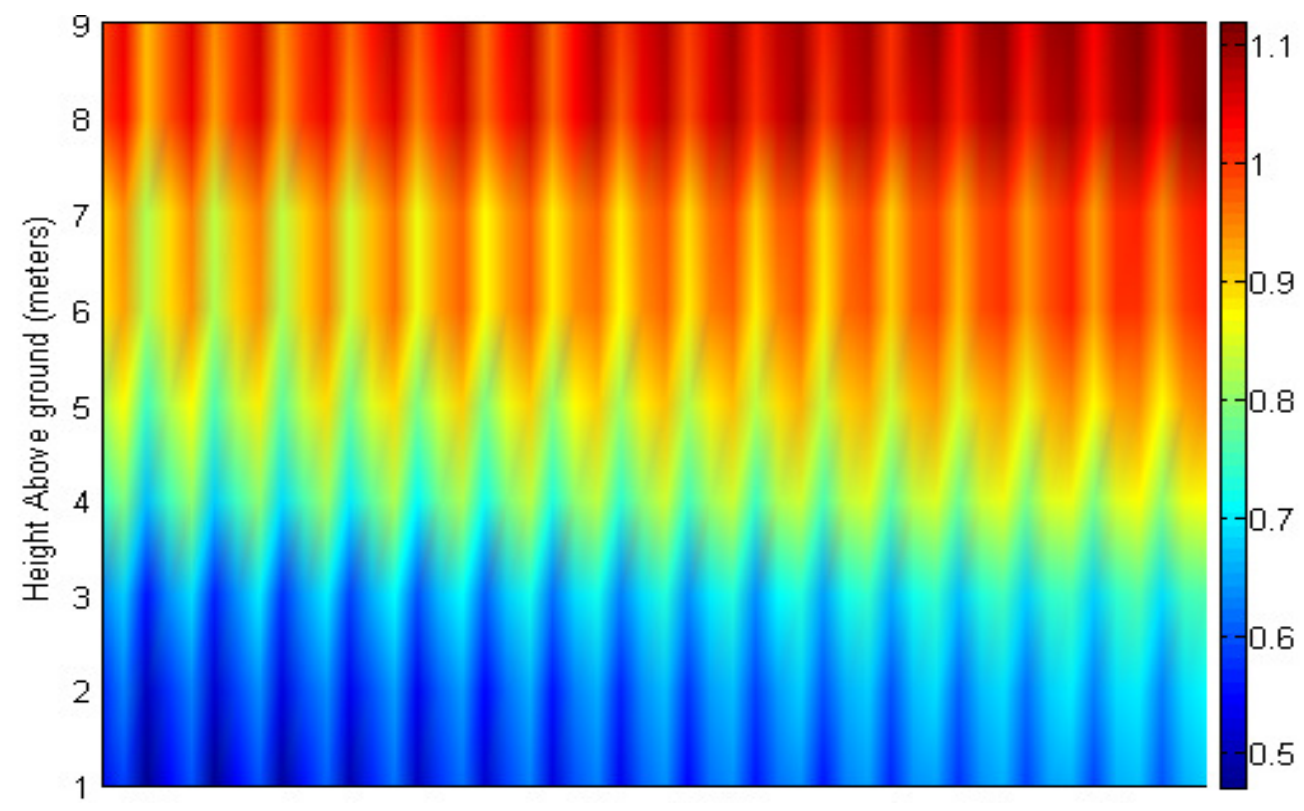

Figure 4.11: EIGER $50 \mathrm{MHz}$ vertical E-field for a $100 \mathrm{~m}$ length underneath middle of $1 \mathrm{~km}$ powerline $(500 \mathrm{~m}-600 \mathrm{~m})$. Color-legend units are $\mathrm{kV} / \mathrm{m}$.

transmission line, using the distributed line currents is appropriate. To do this in ATP, the same cascaded-pi modeling approach as described in Section 4.1 can be used (see Figure 4.1). However, because a lossless line model was used in EIGER (zero internal self inductance), a non-intuitive procedure was used in ATPDraw to control the LCC model building. When a LCC module is built, ATP generates 4 files (.dat, .lis , .pch, and .lib) which are associated with that LCC object. In the Windows ATPDraw version, these files are generated without interruption, and without intermediate control of the user. The .dat file contains the important information used in generating the next three files, and includes the IXFlag. This defaults to 0 which affects the calculation for self inductance. This was described earlier in Section 4.2.1. The IXFlag is not directly controlled by the user in ATPDraw, and is automatically set dependent on whether skin effect is to be included. To get the inductance while ignoring skin effect, the following procedure can be used:

1. Generate the LCC files using ATPDraw.

2. Directly modify the IXFlag in the .dat file (IX $=3$ allows calculation of self induc- 

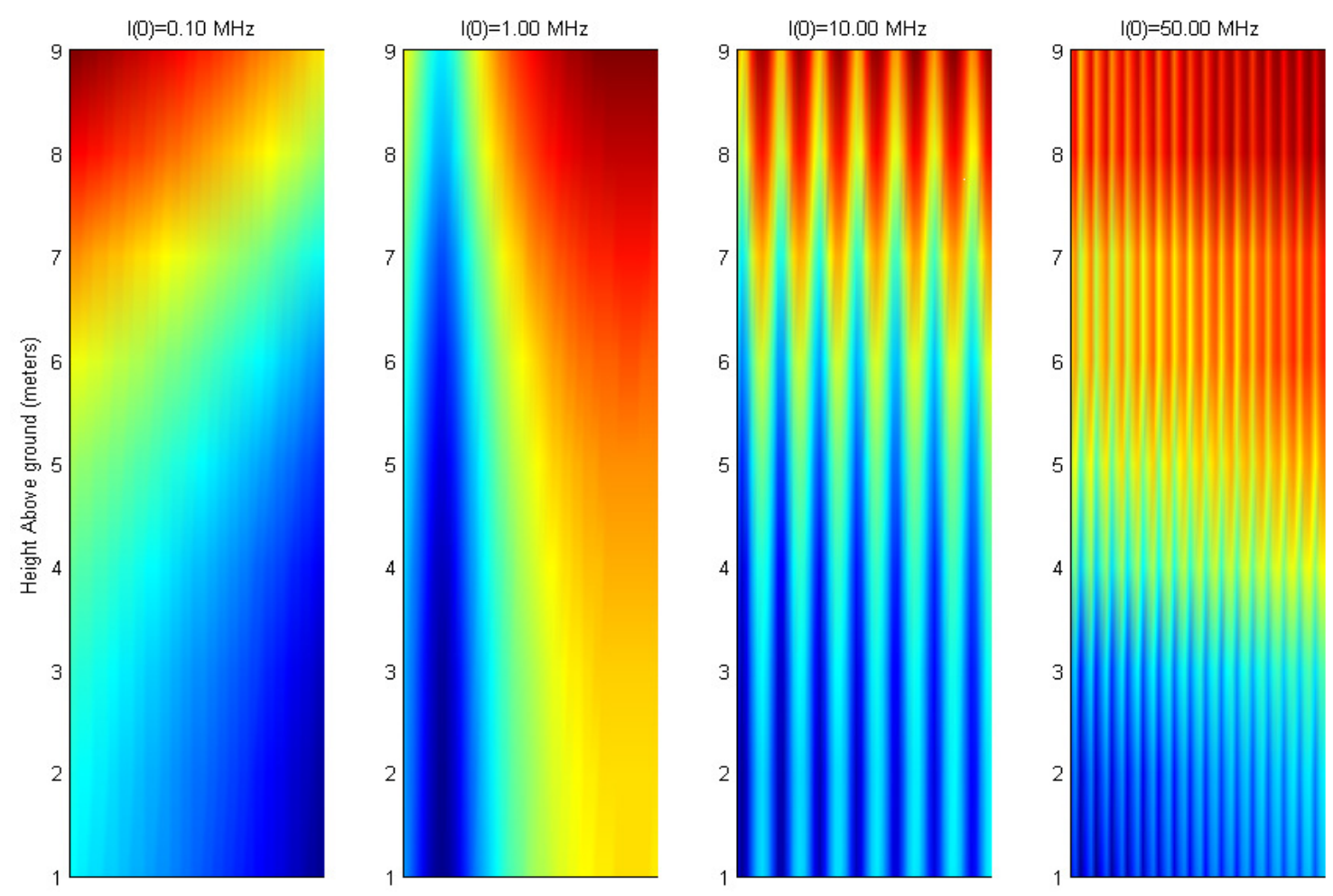

Figure 4.12: Comparison of EIGER vertical E-fields at several frequencies for a $100 \mathrm{~m}$ length underneath middle of $1 \mathrm{~km}$ powerline $(500 \mathrm{~m}-600 \mathrm{~m})$.

tance based on tubular conductor geometry).

3. Use ATPLauncher (available from EEUG download site 3 ) to process the modified .dat file and generate new .lis and .pch files.

4. Finally, the new impedance in the .lis file must be retrieved and manually input to the .$l i b$ file before the ATP simulation is ready to be used with proper impedances.

The results of using this procedure are later addressed in Section 6.2.

\footnotetext{
${ }^{3}$ Licensed users of the ATP are able to download files from the European Users Group site - www.eeug.org. The ATP license is free for nearly everyone and requires only an agreement to the licensing terms.
} 


\subsection{Reconciling a Closer Approximation of ATP Line Con- stants to EIGER}

Given that Carson's derived transmission line equations are inadequate for BPL studies, a new set of equations would be necessary to extend the capabilities of ATP transmission line modeling into those frequency ranges $(2-80 \mathrm{MHz})$. The usefulness of studies pertaining to radiated field patterns is highly dependent on having a software program which can accurately predict the distributed currents of a transmission system while accounting for power system components such as transformers and power electronic devices. In order for the integrated approach for predicting radiated fields described in [1] to be successful, Carson's formulas must be replaced in ATP.

The Carson model based on the quasi-TEM approximation is used widely for low frequency and/or low earth resistivity conditions. Much work has also been done to express exact theory models, such as Wait's full-wave model [26] which leaves out the approximations made by Carson. Wait's approach derives a full solution, however it is computationally challenging to implement. Other researchers have done similar work, but the difficult and rigorous computational implementations cause programs to revert to the Carson model. Marcello D'Amore and Maria Sabrina Sarto introduced a less challenging solution in 1996 [37, 38], based on the full-wave solution of Wait. The proposed model (for single and multi-conductor cases) was touted as an improvement over Carson's formulations because it holds for a wider frequency range. However, D’Amore and Sarto do not account for radiation in their model, making their solution inadequate for BPL studies.

Assuming it is possible, a solution must be derived for the series impedance of multiple conductors over a lossy earth, and it must account for all possible modes of propagation. The broad nature of such formulas would be very advantageous if applied to ATP 
and BPL studies. Though the derived equations would be more computationally challenging than those of Carson, computing power is much improved since Carson's equations were implemented in EMTP in the early 1980's. If implemented as a substitute for Carson's equations in ATP, more general formulas could enable the successful study of BPL networks in a realistic transmission system. Without such equations which are valid into the 2 to $80 \mathrm{MHz}$ range there is little hope in achieving accurately predicted distributed line currents and the associated radiated fields. 


\section{Chapter 5}

\section{Implementation}

A novel approach to prediction of radiated fields in a power system was developed and proposed at the IEEE ISPLC 2010 Conference [1]. This approach involves integration of ATP and EIGER to enable more realistic power system scenarios to be modeled. The approach is depicted in Figure 5.1. ATP is used here, but another EMTP-type software could potentially be used. The generalized process involves first creating a system model in ATP. Next, the model is used to predict the current distribution along transmission lines of interest. These current distributions are then exported for use by EIGER, which finally predicts the radiated fields.

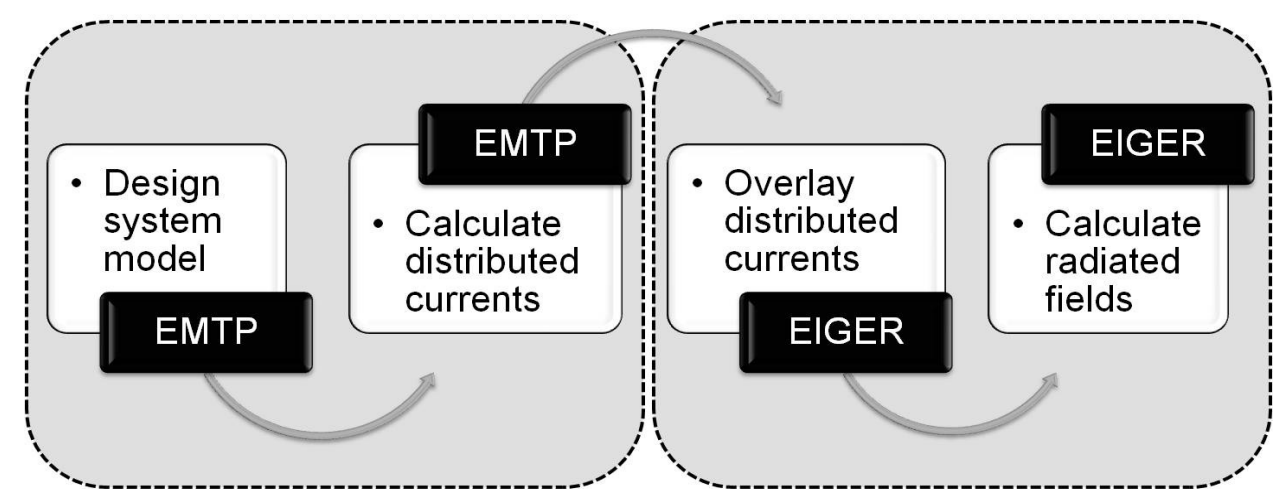

Figure 5.1: Approach to predicting radiated fields from a power system transmission line. 


\subsection{Obtaining High-Frequency Current Distribution Us- ing ATP}

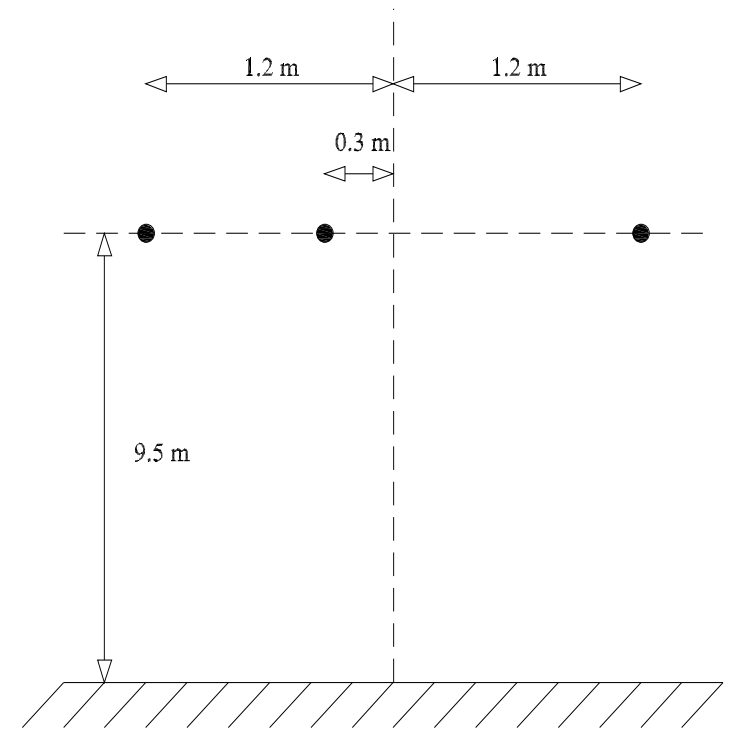

Figure 5.2: Line spacing diagram for test case scenario. Phase A and B are 1.2 and $0.3 \mathrm{~m}$ left of center. Phase $C$ is $1.2 \mathrm{~m}$ right of center.

A test-case transmission line can be built to demonstrate the usefulness of the method for obtaining distributed line currents and radiated fields. An isolated $5 \mathrm{~km}, 3-$ conductor non-transposed line was chosen for study. The flat terrain is a homogeneous

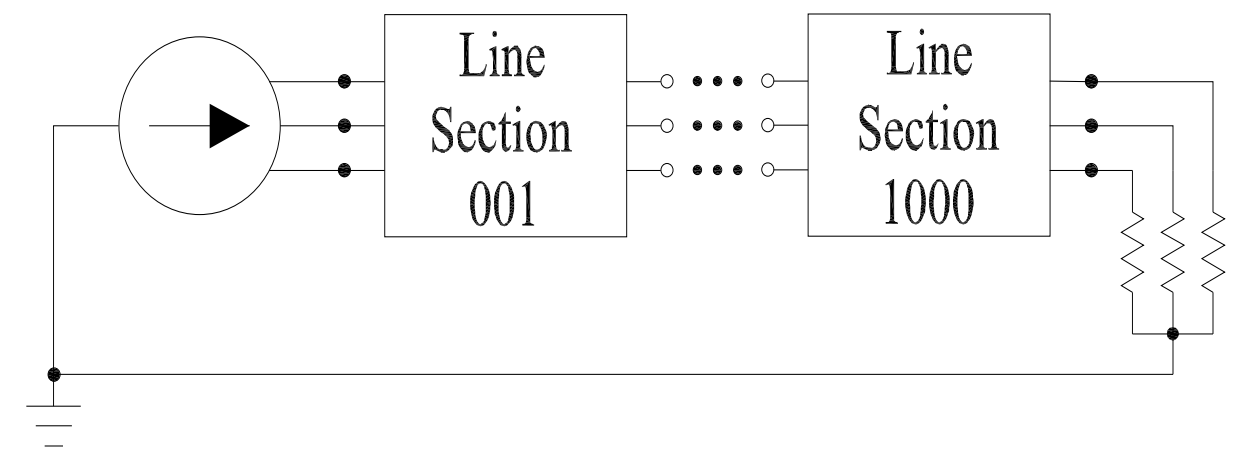

Figure 5.3: Test case scenario with 1,000 pi-sections. 
ground characterized by $\varepsilon=8.0+j 1.0[13]$. Conductor spacing is realistically defined for a standard distribution tower structure. Using the center-pole as a reference (see Figure 5.2), phases A and B are left of center by 1.2192 and 0.3048 meters respectively. Phase $\mathrm{C}$ is right of center by 1.2192 meters. The conductors have a height of 9.5 meters with 0.75 meter sag and $0.03576 \Omega / \mathrm{km}$ dc resistance. The line was terminated with a small, wye-connected load of $10 \Omega$ for each phase. A current source placed at the sending end supplied a 3-phase sinusoidal current as the injected signal. A frequency scan was then used to determine the current distributions for every 5 meters, with 1,000 pi sections in total, see Figure 5.3 .

\subsection{Predicting Radiated Fields with EIGER}

As described in Chapter 3, the ideal case would be for radiated fields from BPL sources to be predicted entirely from EIGER (or other electromagnetics programs). However, transmission lines contain passive and active devices for power distribution control which cannot easily be built in EIGER. Therefore, the EIGER source code was modified to accept the external ATP current distribution (graphically depicted in Figure 5.4). Without this modification, the user would be required to accept a current distribution from a voltage or current source and approximate transmission line devices with lumped parameters; the result would be decreasing accuracy with increasing frequency. Given a BPL current distribution calculated from ATP, the complex current is interpolated and substituted for the EIGER transmission line model current file (*.mnh). Executing the modified version of EIGER results in the ATP current distribution, EIGER model geometry, and terrain information being numerically combined into a Green's Function [13] which is then used to calculate the BPL radiated electric and magnetic fields. Using EIGER is beneficial for several reasons; 1) the field predictions are valid into the $\mathrm{GHz}$ range, 2) one can account for the

presence of a lossy and inhomogeneous earth and 3) geological terrain information (which might otherwise alter predicted BPL fields) can also be included in the EIGER model as 


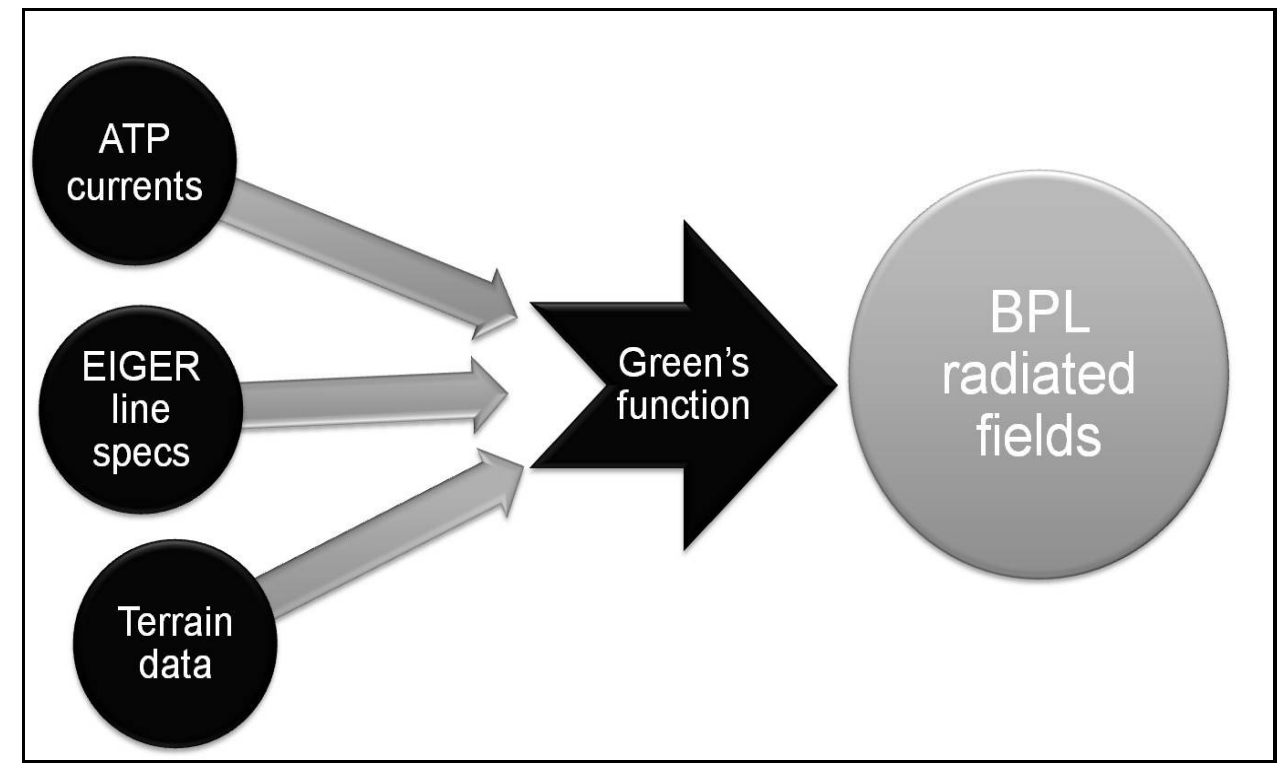

Figure 5.4: EIGER Process

dielectric bodies.

\subsection{Frequency-Dependent Transmission Line Implemen- tation}

As mentioned in Chapter 4, ATP transmission line modeling suffers from limitations of Carson's equations. Furthering the field of BPL studies is dependent first on derivation of an improved set of equations. Assuming a set of equations satisfying the modeling needs is achieved, implementation becomes the next hurdle for research. Implementation possibilities of such formulas are limited outside of hard-coding (ie., internal source code modification). One possibility is to calculate transmission line parameters externally or to implement through ATP's MODELS language. However, there is the issue of how to get these parameters into ATP. The very tedious method of inserting line parameters into a cascaded-pi model (described in Section 4.2.2) is one possibility. However, this can only be done for a single frequency per simulation model. 
It would be more useful to have a model with frequency dependence that could accurately predict power system and BPL performance. One of the newest transmission line models available in ATP is the NODA model [11]. The NODA line model parameters are dumped to a file (which can be modified) which is read by ARMAFIT. Other models do not read in files that can be modified. Another potential method of implementation would be utilizing an external vector-fitting program, which could be used to generate a very high-order RLC equivalent circuit to represent the frequency-dependent transmission line.

\subsubsection{NODA Line Constants with External Modifications}

The NODA model was introduced by Taku Noda [11]. The NODA model differs from the other ATP models in that the calculations are made directly in the phase domain, therefore eliminating approximation errors caused by the use of the transformation matrix. The characteristic admittance and the deformation coefficients are fitted through rational functions. Time domain convolutions are replaced by an ARMA (Auto-Regressive Moving-Average) model that minimizes computation. The modeling of a transmission line using the NODA model normally requires the following two steps:

1. Calculation of the frequency-dependent line parameters (frequency data) of the transmission line using the LCC supporting routine in ATP. The result is written in a .AFT file (ARMAFIT file).

2. Fitting the frequency data (stored in .AFT file) for the time-domain realization of the frequency dependence. This procedure is performed by an independent fitting program ARMAFIT. The result is written in .PCH file ("punch" output file).

ARMAFIT is independent of ATP, meaning ATP is not necessary for calculating the line parameters used in the .AFT file. Frequency data prepared by a user-made line 
constants calculation program can be fitted using ARMAFIT, making it quite useful. In order to use externally implemented formulas to model a frequency dependent transmission line with the NODA ATP model, the following procedure can be used 1 :

1. Formulate the frequency dependent parameters of the transmission line using the derived formulas for series impedance (external to ATP).

2. Build the transmission line model in the NODA LCC module.

3. Run the LCC module to create the transmission line files (.AFT, etc...). In this step, a modification to the batch file that runs ARMAFIT is required. The .BAT file would require a "stop" command before the ARMAFIT routine is called. Otherwise ATPDraw would delete the intermediate. $A F T$ file as a housekeeping procedure.

4. While in "stop" mode, the .AFT file can be retrieved and the NODA $Z$ and $Y$ data can be replaced manually with the D'Amore-Sarto frequency data.

5. Exit the "stop" mode, allowing ARMAFIT to create a time-domain realization of the frequency dependent transmission line. The output, again, is a .PCH file.

With the above procedure, a new set of formulas can be used to represent a line model for a transient simulation. There is, of course, the possibility that the fitting through ARMAFIT can fail, and that the time-domain response can become unstable. The time-step must be pre-determined and other works indicate that small time steps can produce unstable responses. The model parameters can also require fine-tuning of the fitting parameters. In all, however, the NODA implementation described in this section is a good first step in extending the ability of ATP to model transmission lines at BPL frequencies.

\footnotetext{
${ }^{1}$ It should be noted that an obvious precursory step to this procedure is necessary - the formulas must be implemented in a coding environment (eg. - Matlab, C++, Python) in order to produce the frequency-dependent (3-dimensional) $Z$ and $Y$ matrices.
} 


\subsubsection{External Vector Fitting \& Black Box Model}

A second implementation process would be similar to the NODA method, but would not utilize the ARMAFIT feature. A Norwegian scientist named Gustavsen developed a vector fitting technique [39] which later resulted in a set of Matlab routines [40] utilizing rational functions to approximate a frequency dependent matrix into an equivalent electrical network. The set of routines can be used to fit matrices whose frequency dependent elements have been determined from either experimental data or from calculations. A particularly useful facet of this set of programs is that the equivalent electrical network can be imported into ATP. These Matlab routines are also publicly available by the developer and SINTEF Energy Research of Trondheim, Norway.

Similar to the NODA method described earlier, this approach requires a predetermined set of frequency dependent series impedance parameters. This vector fitting approach brings the frequency data into the time domain, and has been demonstrated in ap-

plication to transformers [41] and overhead transmission lines [12]. The end results of the matrix fitting include state equation matrices $(A, B, C, D, E)$ and a file containing the equivalent electrical network (a high-order RLC circuit). The matrices $A, B, C, D$, and $E$ define the state equation $Y(s)$ which has the form

$$
Y(s)=Y_{f i t}(s) u(s)=\left(C(s I-A)^{-1} B+D+s E\right) u(s) .
$$

The equivalent electrical network generated by the Matlab routines has the convenience of being imported directly into ATP. The network branches include elements representing relations between each node and ground:

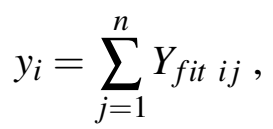




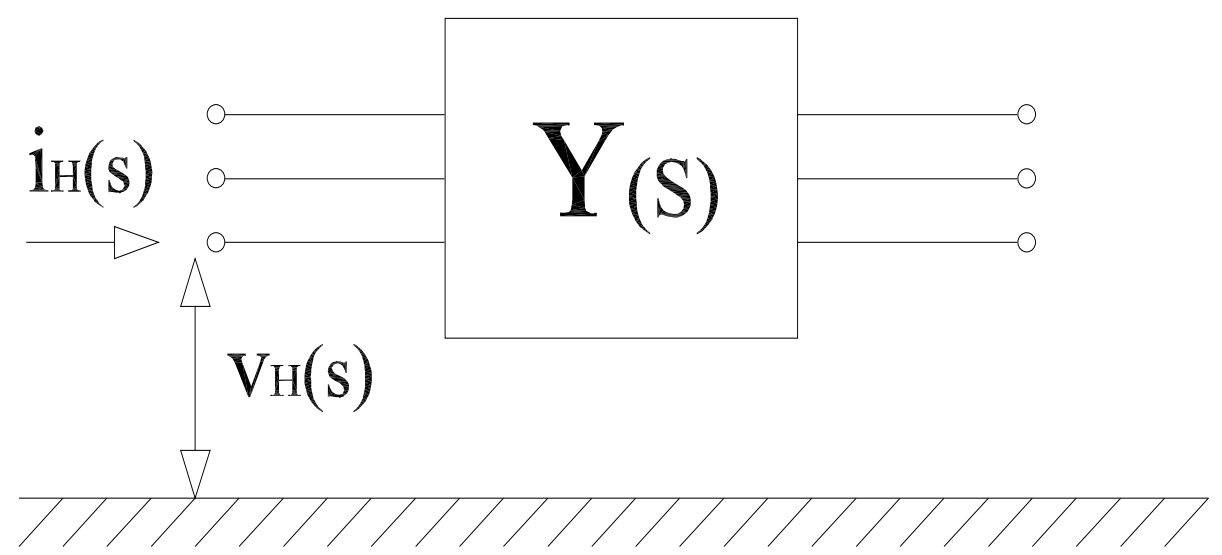

Figure 5.5: A black-box, multi-port, lumped-network model of a power transformer can be created from frequency-dependent nodal voltages and currents.

as well as between all nodes,

$$
y_{i j}=-Y_{\text {fit } i j}
$$

The network branches are converted into the network elements R, L, and C.

A practical example would be useful for better understanding. Consider a set of measured transformer terminal relationships. Transformers are extremely difficult to model at high frequencies because of the existence of many resonance points due to inductive and capacitive effects of the windings, tank, core, etc. Transmission lines are also difficult to model at high frequencies, but for other reasons discussed throughout this paper. Consideration of a transformer example is easier in this case because direct experimental measurements were easier to obtain. The methodology is similar for both cases, however.

The following example utilizes Gustavsen's vector fitting approach as a means of creating lumped networks representing admittance and voltage transfer ratios of a power transformer. These lumped networks are then used in a novel way within ATP to predict the terminal voltages on one winding of a transformer, given known voltages on the other. The 
6x6 admittance matrix $Y$ is related to measured terminal voltages and currents according to

$$
i(s)=Y(s) v(s)
$$

where $v$ and $i$ are frequency-dependent node voltages and currents. In this example, each element of the $Y$ was obtained using an Anritsu ${ }^{T M}$ network analyzer, active voltage probe, and connection board with a wide-band current sensor. Measurements were taken using logarithmically spaced frequency samples between $10 \mathrm{~Hz}$ and $10 \mathrm{MHz}$. Measurements were only taken to complete the upper left quadrant of the $6 x 6$ admittance matrix, as this is the only portion necessary for predicting voltages of an unloaded low-winding, given voltages on the high-winding. That is, in combination with the voltage ratio from low to high windings, the proper impedances are accounted for.

The nodal admittance matrix for the high-side terminals $\left(Y_{H H}\right)$ is the $3 \times 3$ upper left quadrant of the complete 6x6 version. Measurements were taken only for the highside and leaving the low-side open circuited. For each element of the matrix, a separate combination of the 3 high-side terminals is used. For instance, $Y_{11}$ consists of measuring both current and voltage on the $H_{1}$ terminal. The voltage transfer matrix is obtained using similar methods to the $Y$ matrix. Voltage is applied to a terminal of one winding, and the corresponding voltages are measured at the other winding.

The 3x3 matrices obtained for admittance and voltage ratio were appropriately subjected to the rational vector fitting procedure from [40]. Passivity was enforced (as part of the Matlab routines) to ensure stable time domain simulation. Passivity ensures that all eigenvalues of the real part of $Y$ are positive. Finally, the RLC network equivalent circuits for both $Y$ and voltage ratio were generated. These circuit networks can be imported to ATP as library models. The nodes are internal to the model, but can be accessed through specifying node names. Given terminal voltages from the high-side windings as inputs to the $Y$ network, the resulting nodal voltages can be input to the terminals of the voltage ratio 


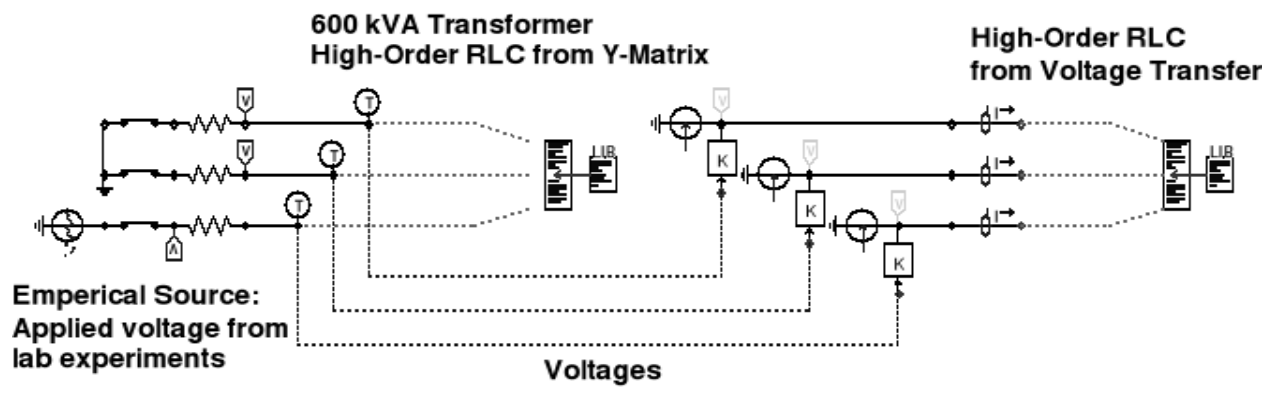

Figure 5.6: $R L C$ circuits in ATP from circuit networks and setup for impulse measurement.

model. Both models are internally grounded three-port networks. If the resulting voltages from the admittance model are used as voltage inputs for the respective terminals of the voltage ratio model, the currents flowing in the voltage ratio model terminals effectively represent the induced voltages for the low-side of the transformer. Consider the case of a 2-winding transformer; using Equation 5.3.2 and partitioning $Y$ into $3 \times 3$ blocks, we get:

$$
\left[\begin{array}{c}
I_{H} \\
I_{L}
\end{array}\right]=\left[\begin{array}{ll}
Y_{H H} & Y_{H L} \\
Y_{L H} & Y_{L L}
\end{array}\right]\left[\begin{array}{c}
V_{H} \\
V_{L}
\end{array}\right],
$$

which results in

$$
\begin{aligned}
V_{H L} & =-Y_{H H}^{-1} \times Y_{H L}, \text { and } \\
V_{L H} & =-Y_{L L}^{-1} \times Y_{L H} .
\end{aligned}
$$

The ATP implementation circuit is shown in Figure 5.6, 


\section{Chapter 6}

\section{Results}

\subsection{Summary of ATP-EIGER Radiation Model}

Using the line description from Section 5.1, the distributed line currents were obtained in ATP. Shown in Figure 6.1 is the distributed current along one conductor as a function of line distance for a $500-\mathrm{kHz}$ injected signal. Note that the figure shows magnitudes only, and that a large source current was used for this demonstration. Though BPL

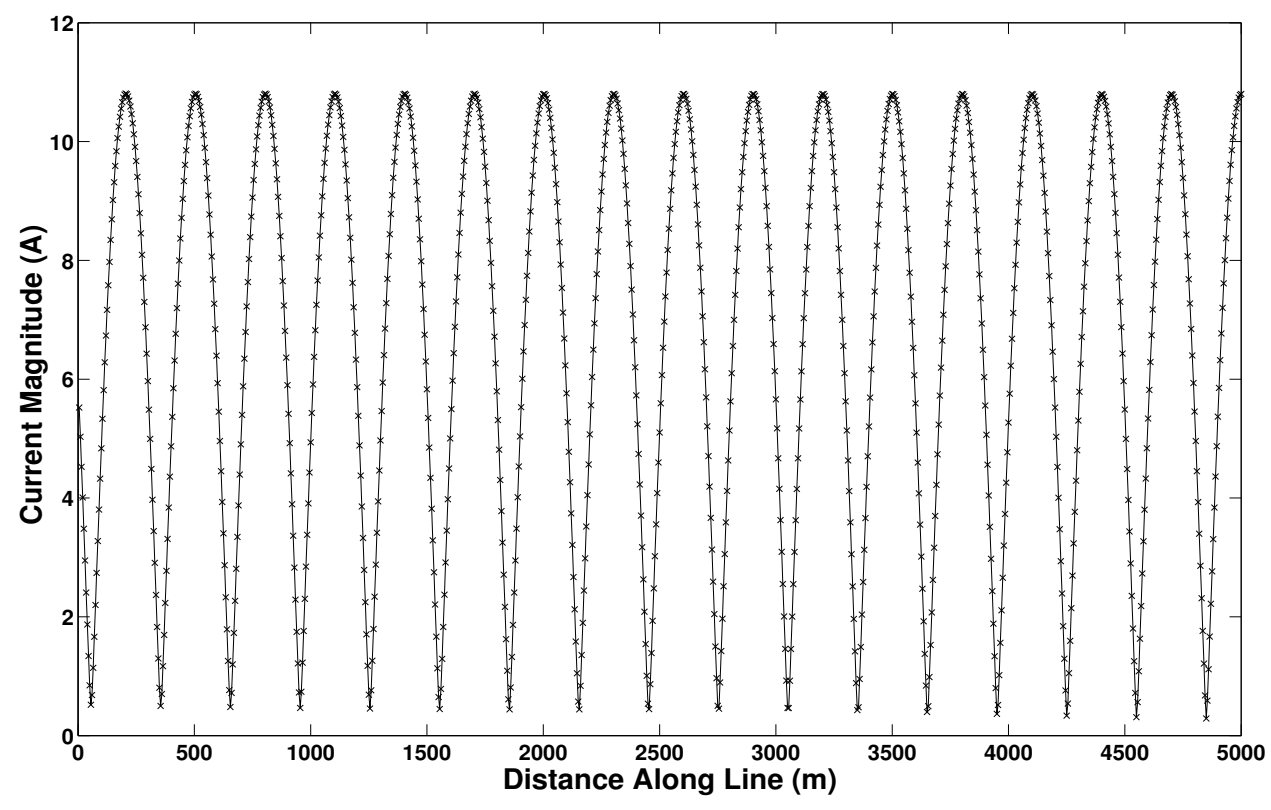

Figure 6.1: $500 \mathrm{kHz}$ ATP current distribution along transmission line. 
systems would typically use smaller signals, the process for determining the currents and resulting fields will be the same as in this example. Additionally, BPL frequencies would be much higher than shown here, but the limits of Carson's formulas prevented use of a higher frequency. In general, the process for obtaining these results is what is important to understand initially. Carson's equations must be updated with enhanced equations before ATP is able to produce reliable results at higher frequencies.

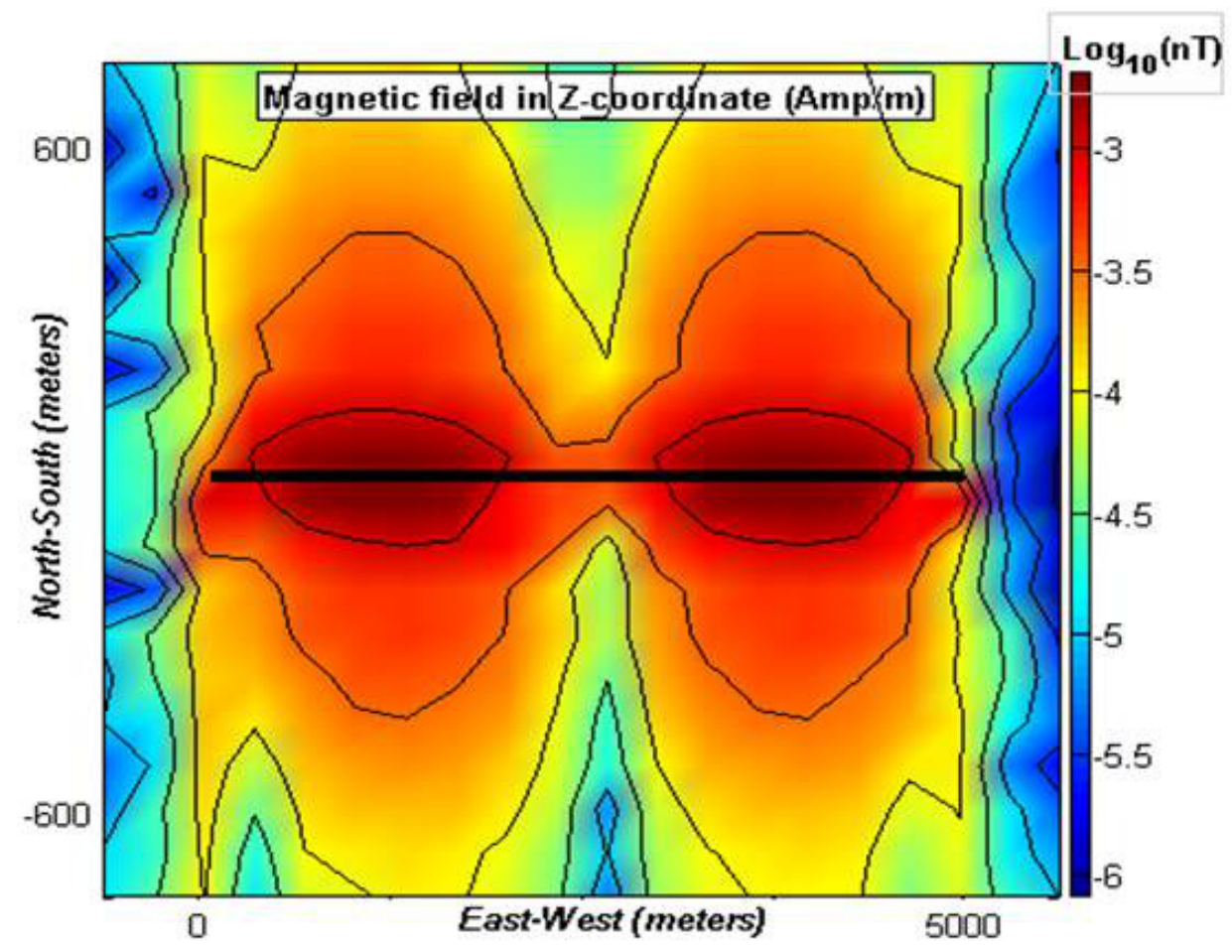

Figure 6.2: Magnitude of radiated vertical magnetic field at altitude of $50 \mathrm{~m}$.

The 5-km transmission line from Section 5.1 was also built into the EIGER model. An ASCII file of the real and imaginary transmission line currents as a function of line distance (for each phase) is created from the ATP line current data of Figure 6.1, In this case, the far-field patterns are not calculated from EIGER, but rather a series of near field points due to the large wavelengths at these frequencies; about 100 meters from ACSR wire with velocity of 0.33 the speed of light at $1 \mathrm{MHz}$ [13]. The fields for this test case are arbitrarily 
calculated at 50 meters above the transmission line in a constant altitude plane, although fields can be calculated in any volume. Figure 6.2 illustrates the results for the amplitude of the vertical magnetic field (the black line represents the transmission line). Note that while Figure 6.1 shows that impedance mismatches at the transmission line boundary set up a standing wave for the current distribution with the number of nodes proportional to the frequency, the radiated fields incorporate the radiation efficiencies of the transmission lines. In essence, the current distribution and radiation efficiency are convoluted.

\subsection{Summary of Carson vs EIGER}

In Section 4.2.2 a transmission line was described and characterized by its radiated fields using EIGER. A modeling process involving ATP was then outlined for obtaining the current distributions down an identical transmission line. Using this procedure, Figures 6.3 and 6.4 were prepared. The plots show the distributed line currents of the 1-km transmission line used for the electric field plots shown earlier (100-meter line sections were included in the E-Field plots).

For the results shown in Figure 6.3, the frequency of signal injected into the sending end $(\mathrm{x}=1000 \mathrm{~m})$ is varied while keeping the earth resistivity constant $(100 \Omega-m)$. The receiving end is open-circuited. Though the results are difficult to interpret, the trend is that the ATP results are similar to the EIGER results until higher frequencies are reached. This was expected, recalling from Section 3.2 and Figure 3.2 that the critical frequency for $\rho=100 \Omega-m$ would be around $180 \mathrm{MHz}$. This would place the potential range of inaccurate ATP results around $f_{\min }=18 \mathrm{MHz}$. For the $50-\mathrm{MHz}$ traces, EIGER results clearly shows a greater attenuation of line currents than that of ATP. The higher frequency traces appear to have a lower initial value at the sending end. In fact, the starting values are the same for each case, though they decrease very quickly resulting in what appears to be a static offset. There are two important factors that contribute to the difference in results 


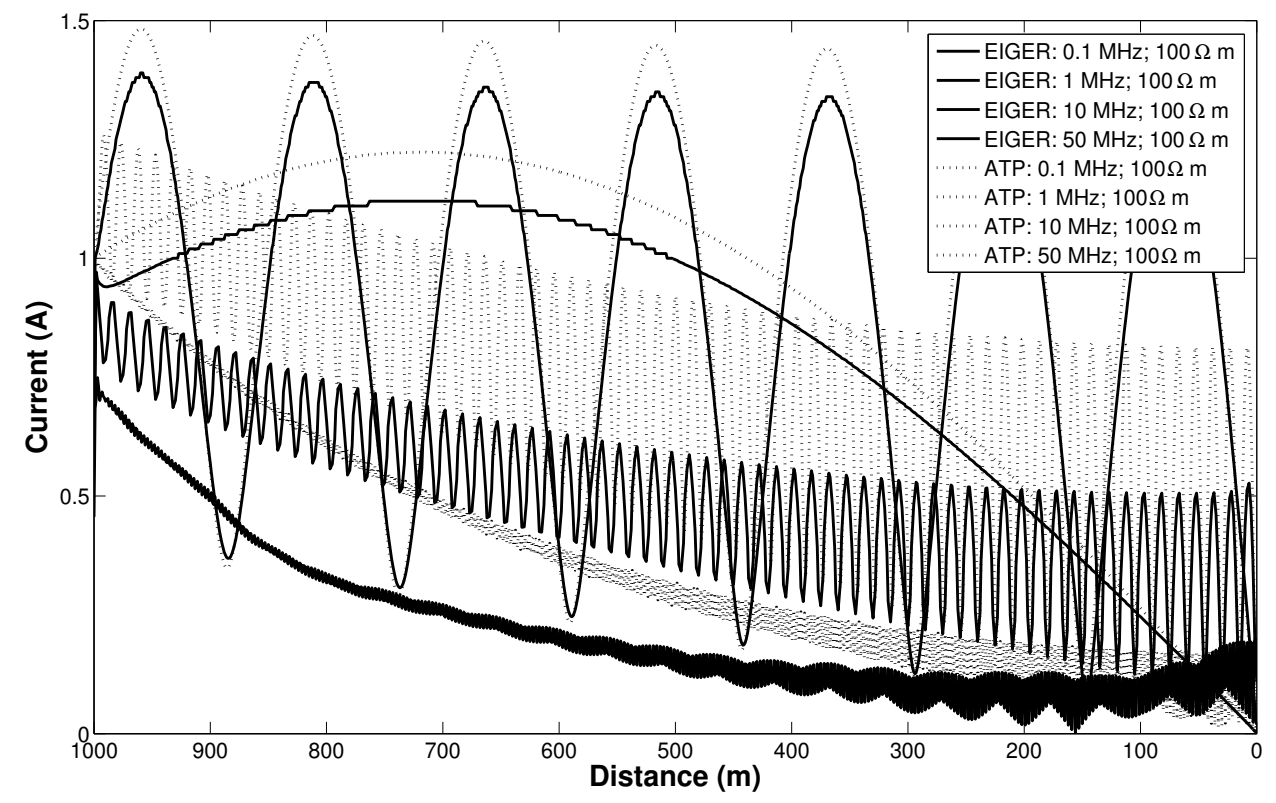

Figure 6.3: ATP and EIGER current distributions for fixed earth resistivity.

between ATP and EIGER:

- Carson's formulas do not account for capacitive ground currents. This was described in Chapter 3, where Figure 3.2 was used to depict the relationship between frequency and resistivity in terms of earth behavior. At higher frequencies (see the $\mathrm{MHz}$-range traces) there are capacitive currents in the earth which are unaccounted for by Carson's equations. The EIGER results do include the capacitive currents, thus the sharp decline in signal amplitude.

- Carson's formulas and the Line Constants models do not account for radiation losses along the transmission line, also related to capacitive effects, which EIGER does account for. Therefore, the ATP results are expected to be higher in amplitude, even at frequencies below the $\mathrm{MHz}$ range.

For the results shown in Figure 6.4, the earth resistivity is varied while keeping the frequency of injection signal constant $(1 \mathrm{MHz})$. In this case, the trend is that the ATP results are similar to the EIGER results until higher earth resistivity is reached. This was expected, recalling from Section 3.2 and Figure 3.2 that the critical frequency boundary is inversely proportional to resistivity $\left(f_{\text {critical }}=1 /\left(2 \pi \varepsilon_{0} \rho\right)\right)$. For the highest shown resistivity, $\rho=$ 


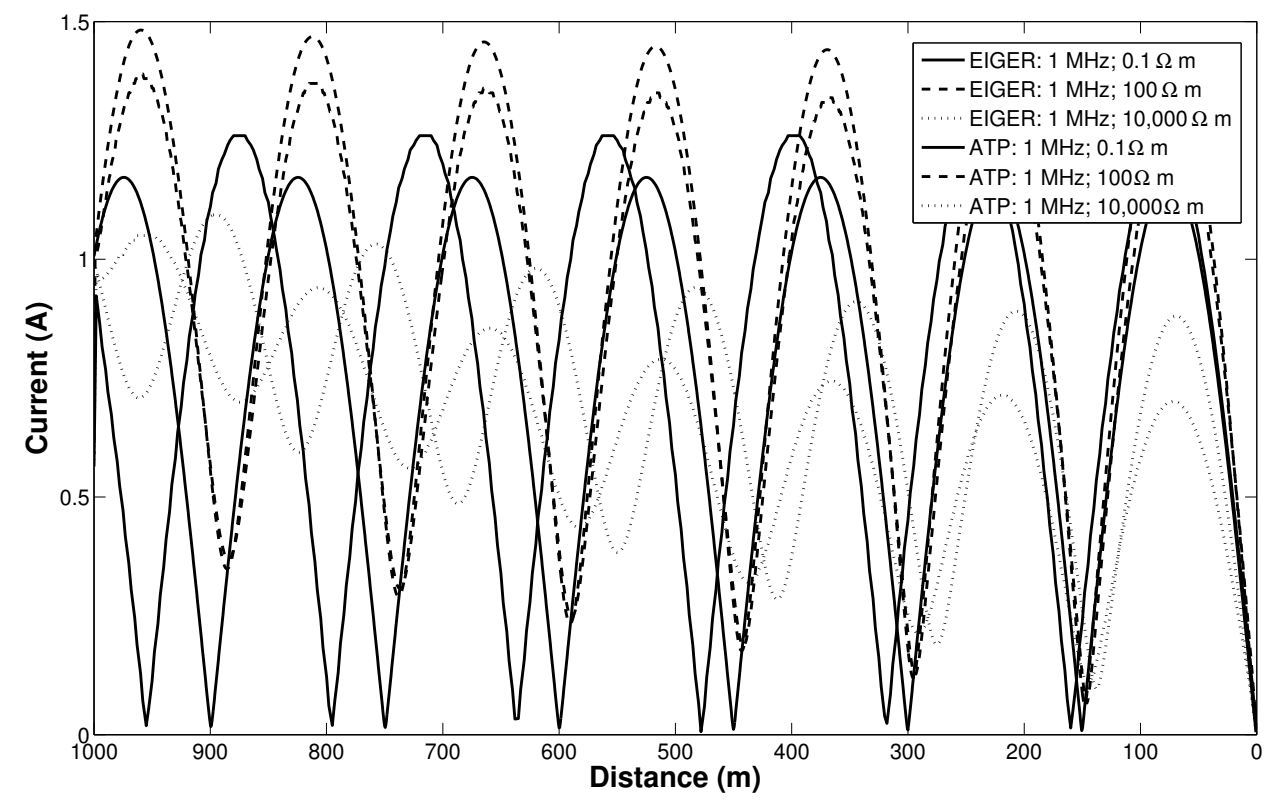

Figure 6.4: ATP and EIGER current distributions for fixed frequency.

$10,000 \Omega-m$, the critical frequency would be around $1.8 \mathrm{MHz}$. This would place the potential range of inaccurate ATP results around $f_{\min }=180 \mathrm{kHz}$. For the $10,000 \Omega-m$ traces, EIGER results clearly shows a greater attenuation of line currents than that of ATP. Again for this figure, there appears to be an offset, especially at the sending end. The traces have the same initial value at the sending end, however the inability of ATP to account for capacitive currents and radiation losses prevents the results from matching closer. The general trend visisble in the plots is the expected result.

\subsection{Summary of Vector Fitting}

Several methods of implementing more valid formulas were discussed in Chapter 5. In order to show the accuracy of the lumped equivalent network produced by the vectorfitting technique discussed in Section 5.3.2, three laboratory impulse testing scenarios were performed and reproduced through simulation with ATP. The testing setup is shown in 
Figure 6.5. The scenario chosen utilizes a transformer, however, this method can be equally applied to transmission lines. The use of a transformer here demonstrates the generality of the method.

\subsubsection{Validation of the Model}

In the first scenario, the step impulse was applied directly to the HV3 transformer terminal with zero input resistance $(\mathrm{R}=0)$. The remaining high voltage terminals were shorted to ground while the low voltage terminals remained open. The second and third scenarios were similar to the first, with addition of $30 \Omega$ and $400 \Omega$ input resistances respectively. This verified the ability to connect the model to a circuit and calculate proper low-winding voltages. The impulse test was then replicated in ATP using the high-order RLC circuits derived from admittance and voltage transfer matrices described earlier. Simple series resistors were used to replicate the input impedances from laboratory setup. An empirical voltage source was used, replicating the voltage input during the actual impulse test.

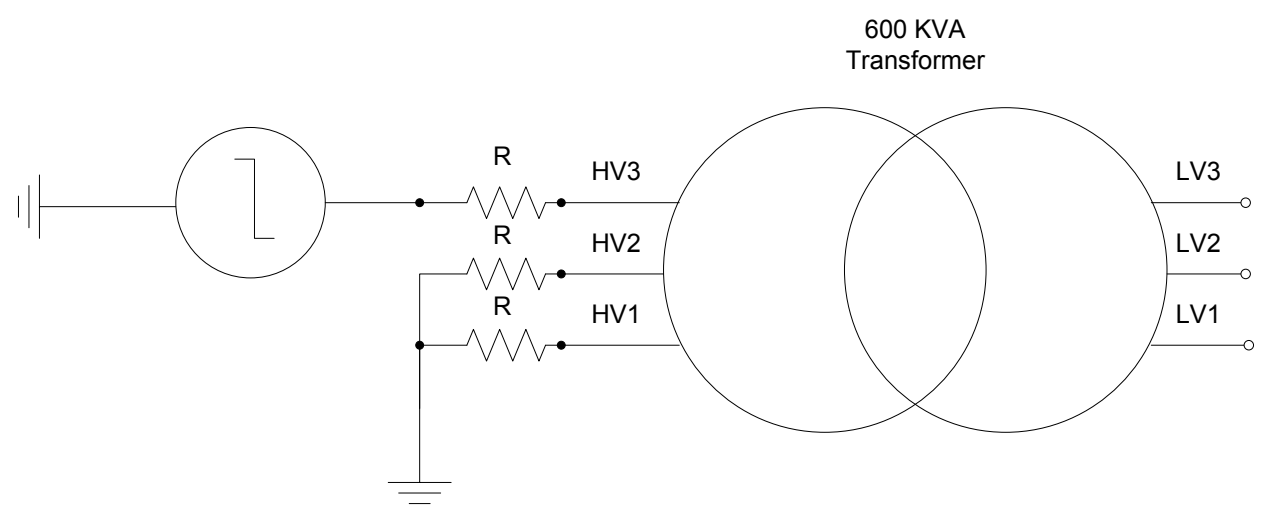

Figure 6.5: Lab impulse testing setup with 600-kVA transformer. 
The first scenario focuses on ensuring the model can accurately predict transient voltages on the low-side terminals given known voltages on the high-side terminals. The impulse voltage applied to high-side terminal 3 (HV3) resulted in a significant response on LV3, and slightly smaller transients on the other terminals. The testing scenario was replicated in ATP, replacing the transformer with the earlier described model. The data from the applied impulse voltage was used as the voltage input to the circuit using an empirical source. The calculated voltages for the low-winding terminals are over-plotted with the measured lab results in Figure 6.6. Note the good agreement between each respective pair of terminals.

The results from Figure 6.6 verify the model is correct for the ideal case without input impedance. Identical tests were then simulated for the 30 and $400 \Omega$ cases, with very good matching results. The results for the $400 \Omega$ simulation are shown in Figure 6.7, Given the matching results for various input impedances during impulse testing, the validity of the implementation method is verified. The combination of frequency dependant admittance

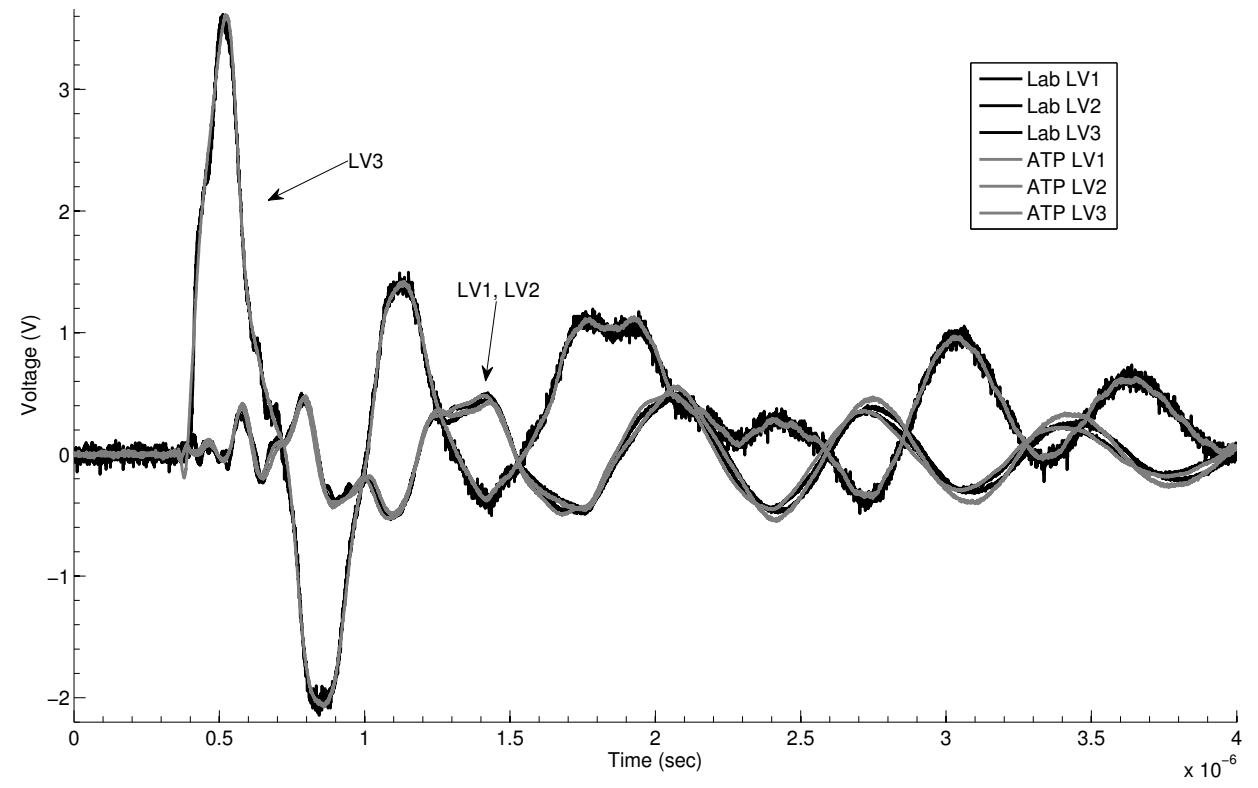

Figure 6.6: Measured and calculated terminal voltages for ideal impulse test. 
and voltage transfer functions accounts for the nonlinear characteristics of the transformer terminal behavior under unloaded conditions. It would now be useful to apply this model to certain realistic scenarios.

\subsubsection{Practical Example: Capacitor Bank Energization}

The first example considered here is that of capacitor bank energization. The ATP circuit is shown in Figure 6.8, with the 600-kVA transformer implemented in the same way as the previous impulse example. This scenario considers a shunt capacitor bank connected between line and ground, considering the capacitor bank and connecting busbars and cables between the bank and a 600-kVA unloaded transformer. The connecting cables are modeled as lossless, distributed parameter cables of $20 \mathrm{~m}$ length, $\mathrm{Z}=30 \Omega$ surge impedance, and a propagation velocity $v=177,000,000 \mathrm{~m} / \mathrm{s}$. A $5 \mathrm{~km}$ overhead line also connects a $22 \mathrm{kV}$ (rms L-L), $50 \mathrm{~Hz}$ source. The conditions under study are the sending and

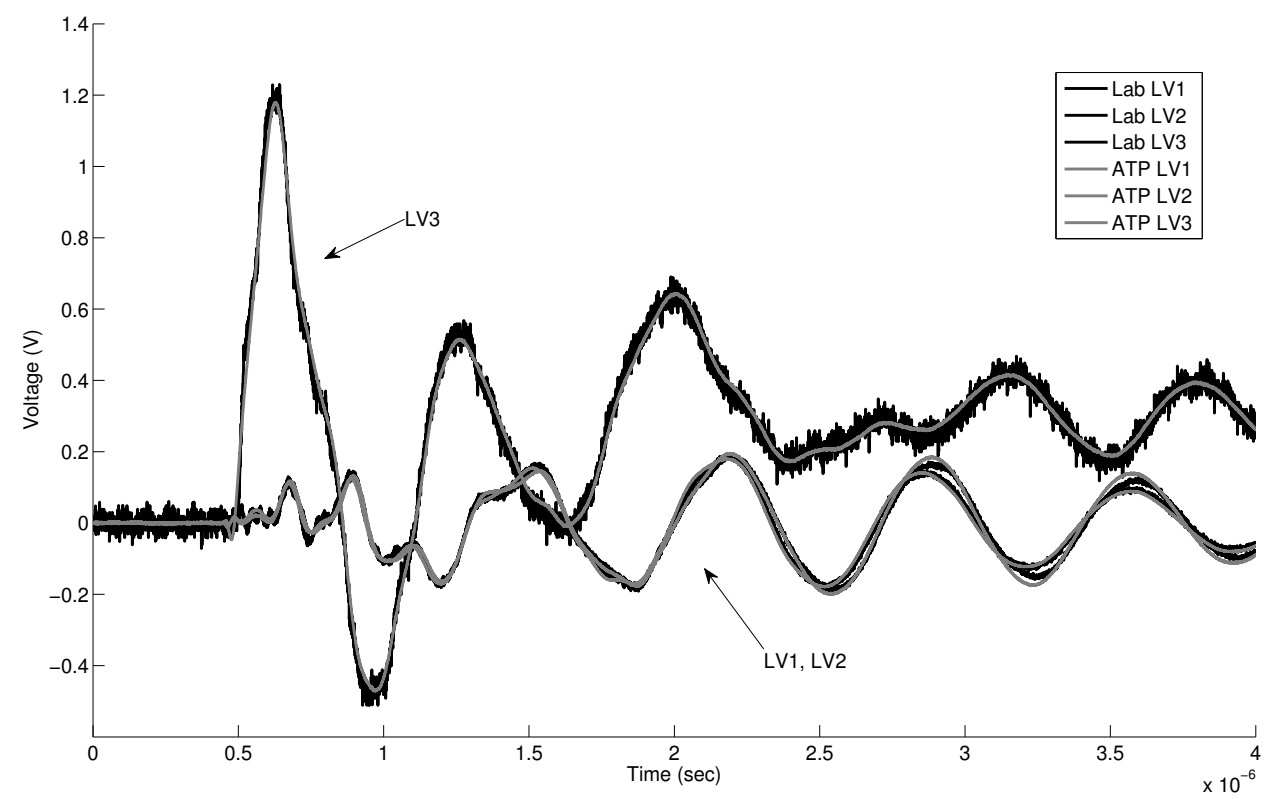

Figure 6.7: Measured and calculated terminal voltages for $R=400 \Omega$ impulse test. 


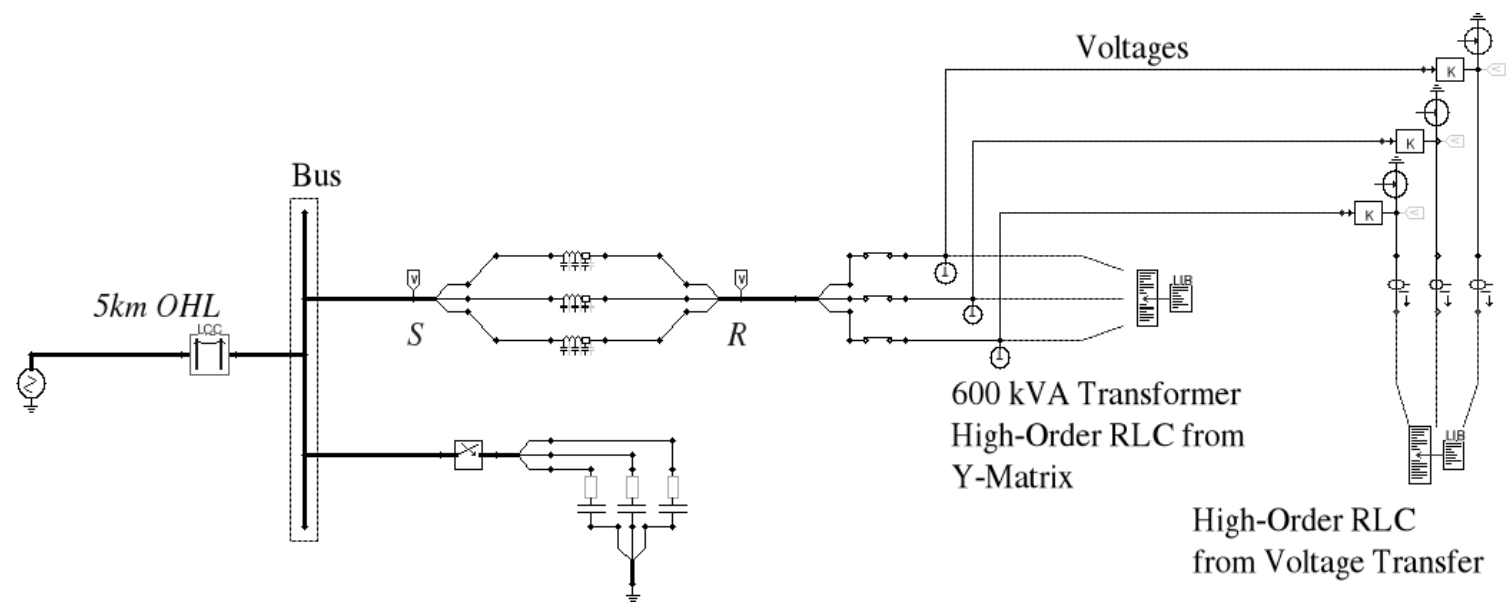

Figure 6.8: Capacitor bank energization example circuit in ATP.

receiving voltages along the connecting cables as well as the low-voltage terminals of the transformer. The results of simulation are shown in Figure 6.9.

The phase- 1 capacitor is switched in at $t=0$, coinciding with voltage peak. Bus volt-

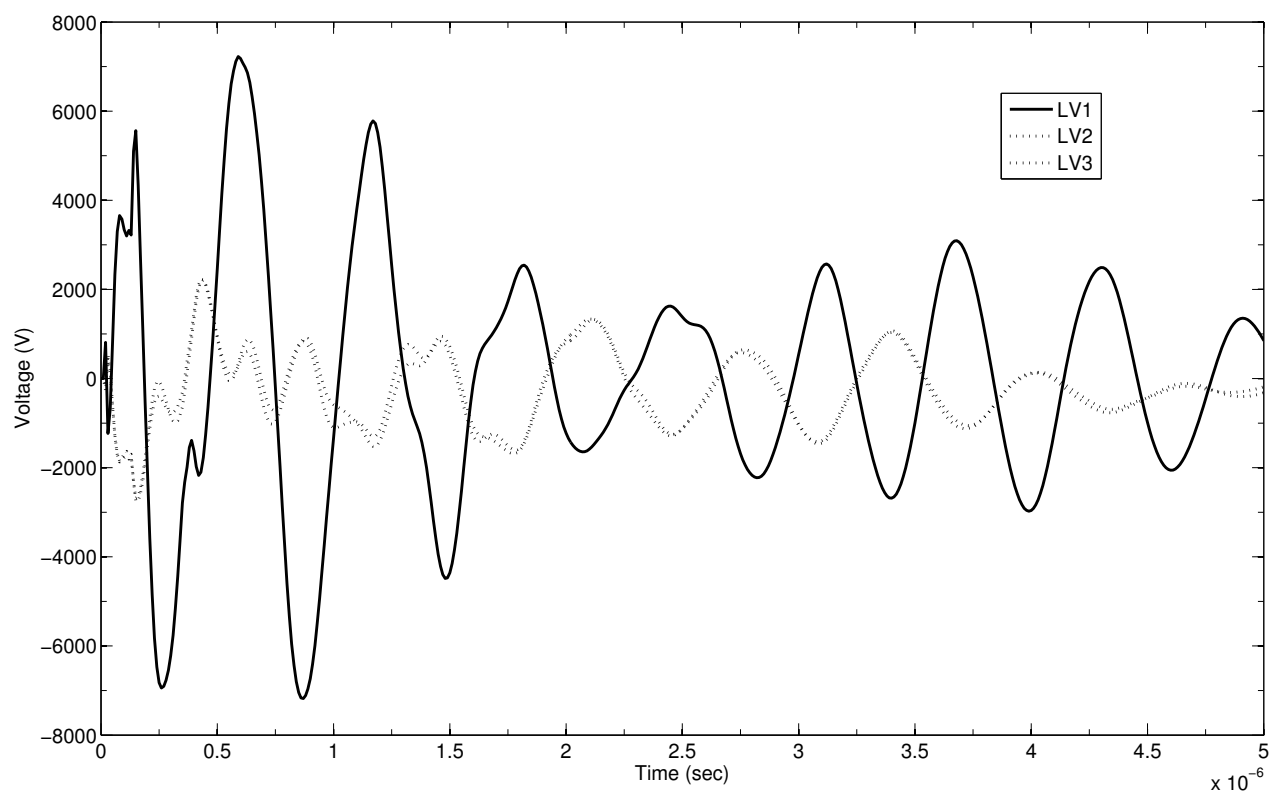

Figure 6.9: Voltages on transformer low-winding terminals for capacitor bank energization test. 


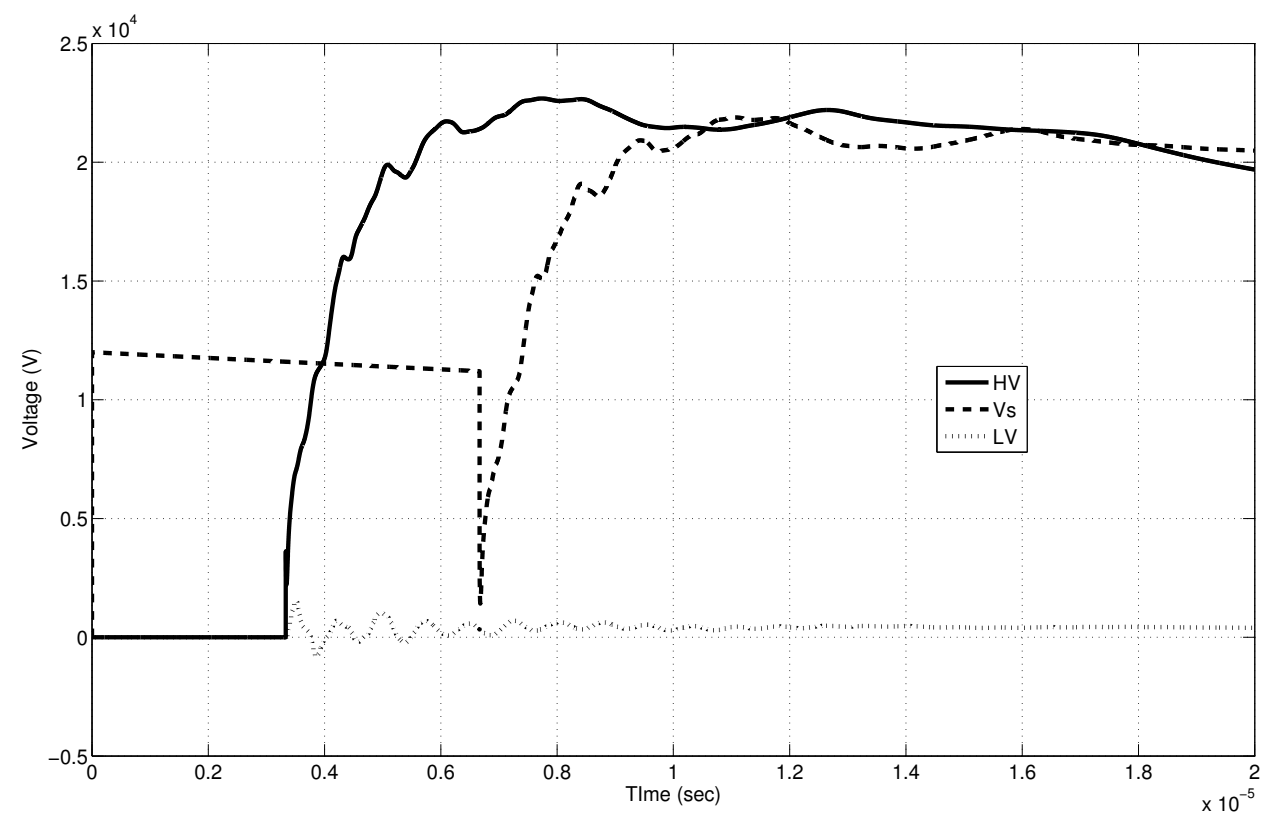

Figure 6.10: Sending voltages and response at transformer.

age Vs1 collapses to $0 \mathrm{~V}$, while the receiving end of the cable sees significant transients after a propagation delay of around $0.11 \mu s$. The transients are also apparent on the low-voltage winding of the transformer. The low-voltage terminal 1 sees the largest overvoltages - on the order of $7 \mathrm{kV}$.

\subsubsection{Practical Example: Lightning Impulse}

The second example considered is that of lightning impulse overvoltages. This is the same circuit used in the previous example, with a few modifications. In this case, the overhead line is modeled using a 3-phase, completely transposed, distributed parameter Clarke line. The lightning surge is injected at the transmission line and is split between the line and a grounded resistor of $400 \Omega$, matching the characteristic impedance of the line. The line is $1000 \mathrm{~m}$ lossless, with $\mathrm{Z}=400 \Omega$ and propagation velocity equal to the speed of light. 
The injected current pulse used had a peak of $60 \mathrm{~A}$, a rise time of $1 \mathrm{~ns}$, and a fall time $100 \mu \mathrm{s}$. The results of the actual simulation are shown in Figure 6.10, The voltage at the sending end of the cable rises almost instantaneously, while the surge propagates down the previously de-energized overhead line to the transformer. This propagation takes approximately $3.3 \mu \mathrm{s}$, at which time there are some reflected and refracted voltages at the interface of transformer and transmission line. As expected, the receiving end (HV terminal) voltages rise to roughly twice that of the sending end, and the effects of the reflected wave on the sending end are visible as well. Again, there are interesting results for the low-voltage terminals, better seen in Figure 6.11, with transient peaks up to $1.6 \mathrm{kV}$.

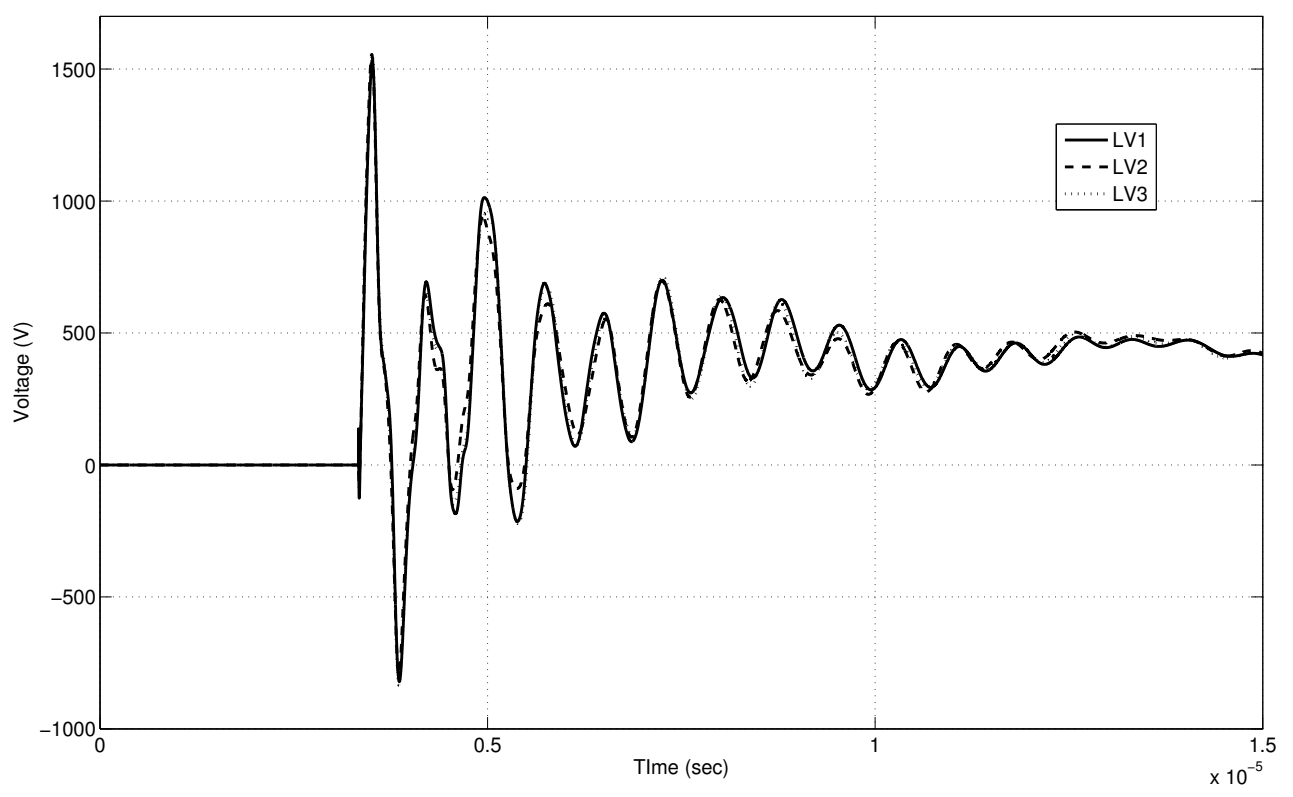

Figure 6.11: Voltages on transformer low-winding terminals for lightning impulse test. 


\section{Chapter 7}

\section{Conclusions and Recommendations}

This final chapter presents the conclusions drawn from this work, and the author's recommendations for future research. Concluding statements are made for 1 ) the use of Carson's formulas in ATP, 2) the ATP-EIGER approach to predicting distributed currents and radiated fields, 3) the potential use of the high-frequency NODA implementation method, and 4) the potential use of the high-frequency Vector Fitting implementation method.

\subsection{Conclusions}

- Conclusions about Carson's formulas used in ATP:

- The series impedance equations derived by Carson are identical in form and behavior to those implemented in EMTP-ATP, but they are not quite identical to those referenced in the EMTP Theory book, which has been attributed to a documentation error.

- Carson's equations for the series impedance of a conductor over a lossy earth have many assumptions which restrict their usefulness for high frequencies or for low conductivity of earth.

- The TEM or quasi-TEM mode is typically the only mode of propagation on transmission lines operated at or below the PLC frequency range.

- Additional modes have the possibility to propagate as operating frequencies extend well beyond those of normal operation. In fact, other modes of propagation begin to appear for an increasing ratio of $h / \lambda, h$ being the wire height above earth and $\lambda$ being the free-space wavelength. 
- ATP transmission line models are not capable of accounting for modes of propagation other than the quasi-TEM mode.

- There are 4 additional modes of propagation possible for a long wire above a lossy ground.

- Transmission line parameters must be recalculated for each value of frequency.

- Conclusions about the ATP-EIGER approach to predicting distributed currents and radiated fields:

- The novel approach described for integrating ATP and EIGER to obtain distributed line currents and radiated fields is an important step toward enabling computational studies of realistic BPL scenarios and for advancing the state of BPL research.

$\circ$ In this approach, EMTP-ATP is used to determine the current distribution of a transmission line. This current distribution is then overlaid onto the electromagnetic EIGER model of the physical transmission line to determine the radiated fields of a BPL system.

- This two-fold system is beneficial because EMTP-ATP can accurately model power electronic devices and control schemes and the components found in power systems (e.g. power transformers, instrument transformers, communication couplers, etc.) which cannot be ignored in these studies. Given this current distribution, EIGER can account for the inhomogeneity commonly found in the earth as well as using dielectric bodies to approximate terrain effects.

- While EIGER is valid into the microwave region (GHz), EMTP-ATP capabilities are limited by the use of Carson's formulas.

- The ATP-EIGER approach is a valid method of incorporating power system components into studies of radiated fields from transmission lines.

- A substitute for Carson's formulas is necessary in order for ATP to be useful for these studies into the BPL (2-80 MHz) frequency region.

- Conclusions about high-frequency NODA implementation method (Section 5.3.1)

- A set of appropriate formulas are able to be coded in a program external to ATP, whereby the program could produce frequency dependent $Z$ and $Y$ matrices.

$\circ$ Only one model is required for an overall frequency range. 
- The NODA line model in ATP can be modified to accept the externally created $Z$ and $Y$ matrices.

- The frequency dependent $Z$ and $Y$ can then be fitted using an Auto-Regressive Moving Average (ARMAFIT) technique.

- It is possible for the derived formulas to be used to represent a line model for a transient simulation in ATP using the NODA setup.

- Conclusions about high-frequency Vector Fitting [40] implementation method:

- Similar to the NODA method, a set of appropriate formulas are able to be coded in a program external to ATP, whereby the program could produce frequencydependent data for the transmission line(s).

- Only one model is required for an overall frequency range.

- Vector Fitting seems suitable for high frequency modeling of multi-port networks, and utilizes Matlab routines which are publicly available by the developer and SINTEF Energy Research of Trondheim, Norway.

- This method utilizes rational functions to approximate a frequency dependent matrix into an equivalent electrical network.

$\circ$ The set of routines can be used to fit matrices whose frequency dependent elements have been determined from either experimental data or from calculations.

- Vector Fitting brings the frequency data into the time domain, and has been experimentally demonstrated and verified in this paper through application to power transformers.

- The equivalent electrical network is a high-order approximation using lumped RLC branches, and can be directly imported into ATP as a multi-port network.

\subsection{Recommendations}

The following recommendations would be made for future work in continuation of this research topic:

- Continuation of the study of BPL using ATP will require identification of a set of equations to replace those of Carson. The equations must account for all appropriate modes of propagation, with accuracy into the 2 to $80 \mathrm{MHz}$ range. 
- If an appropriate set of equations is identified, the formulas should be verified against EIGER to benchmark the correctness. This can be done by simply modeling a singlefrequency transmission line in ATP (using the cascaded-pi approach from this paper) and comparing the current distribution to that of EIGER.

- Immediate research can begin with a valid set of formulas by testing the two implementation methods described in Chapter 5 .

- A long-term solution may involve permanent code implementation into ATP, but would require help of ATP developers.

- The usefulness of ATP in studying BPL from a power system perspective can finally be realized if this additional work and progress is made.

\subsection{Closing Comments}

There is apparent usefulness of a transmission line model in ATP which is valid for higher frequencies. The current transmission line models used in ATP perform well in the conditions for which they were intended. In the case of those utilizing Carson's equations, this means they perform well (in general) for frequencies below the $\mathrm{MHz}$ range. Studies of BPL and radiated fields have never been successfully performed in an environment that includes the behaviors of a power system (such as studies performed with ATP). More specifically, attempts at these types of studies using ATP had never before been published or publicly documented prior to 2010. The work presented in this thesis includes the first such published attempt [1], which was essentially an early thrust of this research project. The work that followed was a result of the apparent deficiencies of ATP transmission line models at BPL frequencies. As such, the study of BPL using EMTP-type transmission line models with the intent of predicting radiated fields remains in the infant stages. A great amount of groundbreaking and exciting research remains in this field. 


\section{References}

[1] B. A. Mork, N. M. Stenvig, R. M. Nelson, and B. Kirkendall, "Determination of high-frequency current distribution using emtp-based transmission line models with resulting radiated electromagnetic fields," in Power Line Communications and Its Applications (ISPLC), 2010 IEEE International Symposium on, pp. 219 -224, 28-31 2010.

[2] B. A. Mork, D. Ishchenko, X. Wang, A. Yerrabelli, R. Quest, and C. Kinne, "Power line carrier communications system modeling," in International Conference on Power Systems Transients (IPST), 2005, 2005.

[3] R. Olsen and B. Stimson, "Predicting vhf/uhf electromagnetic noise from corona on power-line conductors," Electromagnetic Compatibility, IEEE Transactions on, vol. 30, no. 1, pp. $13-22,1988$.

[4] IEEE Special Publication TP-133-0, "Modeling and analysis of power system transients using digital programs," IEEE Catalog No.99TP133-0, 1999.

[5] GE Power Management, "Relaying communications channels application guide," Publication No. GET-8034, 2001.

[6] A. Greenwood, Electrical Transients in Power Systems. Canada: John Wiley \& Sons, second ed., 1991.

[7] J. Snelson, "Propagation of travelling waves on transmission lines - frequency dependent parameters," Power Apparatus and Systems, IEEE Transactions on, vol. PAS-91, pp. 85 -91, Jan. 1972.

[8] W. Meyer and H. Dommel, "Numerical modelling of frequency-dependent transmission-line parameters in an electromagnetic transients program," Power Apparatus and Systems, IEEE Transactions on, vol. PAS-93, pp. 1401 -1409, Sept. 1974.

[9] A. Semlyen and A. Dabuleanu, "Fast and accurate switching transient calculations on transmission lines with ground return using recursive convolutions," Power Apparatus and Systems, IEEE Transactions on, vol. 94, no. 2, pp. 561 - 571, 1975. 
[10] J. R. Marti, "Accurate modeling of frequency-dependent transmission lines in electromagnetic transient simulations," Power Engineering Review, IEEE, vol. PER-2, no. 1, pp. $29-30,1982$.

[11] T. Noda, N. Nagaoka, and A. Ametani, "Phase domain modeling of frequencydependent transmission lines by means of an arma model," Power Delivery, IEEE Transactions on, vol. 11, no. 1, pp. $401-411,1996$.

[12] A. Morched, B. Gustavsen, and M. Tartibi, "A universal model for accurate calculation of electromagnetic transients on overhead lines and underground cables," Power Delivery, IEEE Transactions on, vol. 14, no. 3, pp. 1032 -1038, 1999.

[13] C. A. Balanis, Advanced Engineering Electromagnetics. Canada: John Wiley \& Sons, first ed., 1989.

[14] R. Olsen, "Electromagnetic fields from power lines," in Electromagnetic Compatibility, 1993. Symposium Record., 1993 IEEE International Symposium on, pp. 138-143, 9-13 1993.

[15] L. Cohen, J. de Graaf, A. Light, and F. Sabath, "The measurement of broad band over power line emissions," in Electromagnetic Compatibility, 2005. EMC 2005. 2005 International Symposium on, vol. 3, pp. 988 - 991 Vol. 3, 8-12 2005.

[16] J.-G. Rhee, E. Rhee, and T.-S. Park, "Electromagnetic interferences caused by power line communications in the hf bands," in Power Line Communications and Its Applications, 2008. ISPLC 2008. IEEE International Symposium on, pp. 249 -252, 2008.

[17] Y. Guo, Z. Xie, and Y. Wang, "A model for $10 \mathrm{kv}$ overhead power line communication channel," in International Symposium on Computer Science and Computational Technology. Proceedings, 2009, Dec. 2009.

[18] S. Tsuzuki, M. Yoshida, and Y. Yamada, "Power-line channel modeling for commonmode signal transmission/suppression," in Power Line Communications and Its Applications, 2005 International Symposium on, pp. 210 - 214, 2005.

[19] W. Q. Luo, S. Y. Tan, and B. T. Tan, "Effects of the ground on power-line communications," Microwave Theory and Techniques, IEEE Transactions on, vol. 53, pp. 3191 - 3198, Oct. 2005.

[20] E. Anatory, N. Theethayi, R. Thottappillil, M. Kissaka, and N. Mvungi, "Broadband power-line communication channel model: Comparison between theory and experiments," in Power Line Communications and Its Applications, 2008. ISPLC 2008. IEEE International Symposium on, pp. 322 -324, 2008.

[21] G. Antonini, A. Orlandi, R. Rizzi, and A. Costanzi, "Systematic analysis of electromagnetic emissions and susceptibility in bpl system," in Power Line Communications 
and Its Applications, 2007. ISPLC '07. IEEE International Symposium on, pp. 5 -10, 2007.

[22] P. Amirshahi and M. Kavehrad, "Medium voltage overhead power-line broadband communications; transmission capacity and electromagnetic interference," in Power Line Communications and Its Applications, 2005 International Symposium on, pp. 2 $-6,2005$.

[23] C. R. Paul, Analysis of Multiconductor Transmission Lines. Canada: Wiley-IEEE Press, second ed., 2007.

[24] F. M. Tesche, M. Ianoz, and T. Karlsson, EMC Analysis and Computational Models. Canada: Wiley-Interscience, first ed., 2007.

[25] R. Olsen, "Power-transmission electromagnetics," Antennas and Propagation Magazine, IEEE, vol. 36, pp. 7 -16, Dec. 1994.

[26] J. Wait, "Theory of wave propagation along a thin wire parallel to an interface," Radio Science, vol. 7, pp. $675-679$, Jun. 1972.

[27] R. Olsen and D. Chang, "New modal representation of electromagnetic waves supported by horizontal wire above dissipative earth," Electronics Letters, vol. 10, pp. 92 -94, Apr. 1974.

[28] J. Carson, "Wave propagation in overhead wires with ground return," Bell System Technical Journal, vol. 5, pp. 539-554, 1926.

[29] C. Paul, "A comparison of the contributions of common-mode and differential-mode currents in radiated emissions," Electromagnetic Compatibility, IEEE Transactions on, vol. 31, pp. 189 -193, May 1989.

[30] H. Dommel, Electromagnetc Transients Program (EMTP) Theory Book. Bonneville Power Administration, Portland, OR., 1981.

[31] R. Olsen, J. Young, and D. Chang, "Electromagnetic wave propagation on a thin wire above earth," Antennas and Propagation, IEEE Transactions on, vol. 48, pp. 1413 -1419 , Sept. 2000.

[32] H. Kikuchi, "Propagation characteristics along a dielectric coated cylindrical conductor above the ground," Proceedings of the IEEE, vol. 66, no. 3, pp. $351-352,1978$.

[33] A. Semlyen, "Ground return parameters of transmission lines an asymptotic analysis for very high frequencies," Power Apparatus and Systems, IEEE Transactions on, vol. PAS-100, pp. 1031 -1038, Mar. 1981.

[34] A. Sommerfeld, "Propagation of waves in wireless telegraphy," Ann. Phys., vol. 81, pp. 1367-1153, 1926. 
[35] G. Goubau, "Surface waves and their application to transmission lines," J. Appl. Phys., vol. 21, pp. 1119-1128, 1950.

[36] E. Scharlemann, An EMTP Theory Book Correction, LLNL-TR-450052. Lawrence Livermore National Laboratory, Livermore, CA., 17 August 2010.

[37] M. D'Amore and M. Sarto, "Simulation models of a dissipative transmission line above a lossy ground for a wide-frequency range. i. single conductor configuration," Electromagnetic Compatibility, IEEE Transactions on, vol. 38, pp. 127 -138, May 1996.

[38] M. D'Amore and M. Sarto, "Simulation models of a dissipative transmission line above a lossy ground for a wide-frequency range. ii. multiconductor configuration," Electromagnetic Compatibility, IEEE Transactions on, vol. 38, pp. 139 -149, May 1996.

[39] B. Gustavsen and A. Semlyen, "Rational approximation of frequency domain responses by vector fitting," Power Delivery, IEEE Transactions on, vol. 14, no. 3, pp. $1052-1061,1999$.

[40] B. Gustavsen, "Computer code for rational approximation of frequency dependent admittance matrices," Power Delivery, IEEE Transactions on, vol. 17, no. 4, pp. 1093 $-1098,2002$.

[41] B. Gustavsen, "A hybrid measurement approach for wideband characterization and modeling of power transformers," Power Delivery, IEEE Transactions on, vol. 25, no. 3, pp. $1932-1939,2010$. 


\section{Appendix A}

\section{Programming Code}

\section{A.1 Carson and EMTP Equations in Python, C++}

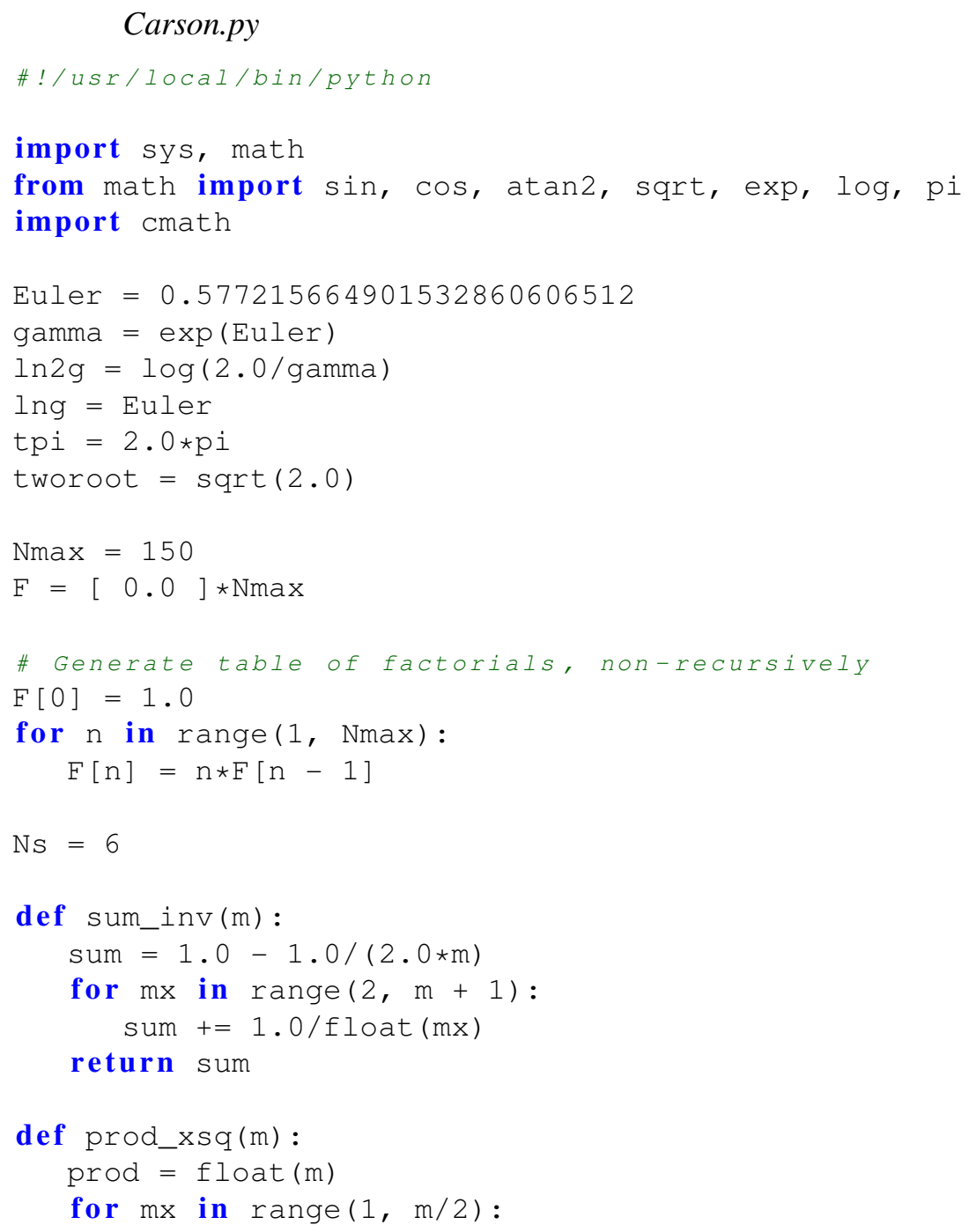




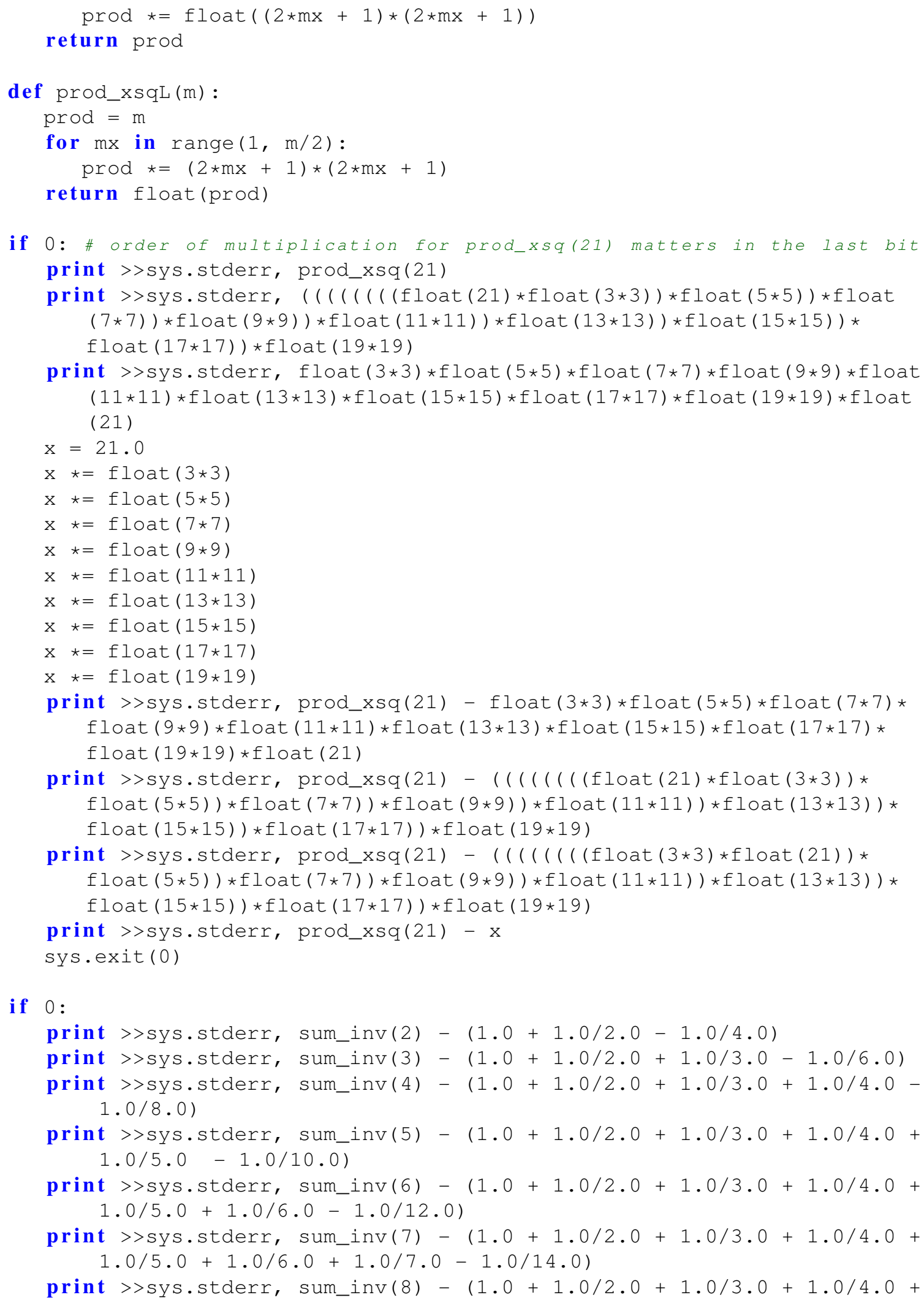




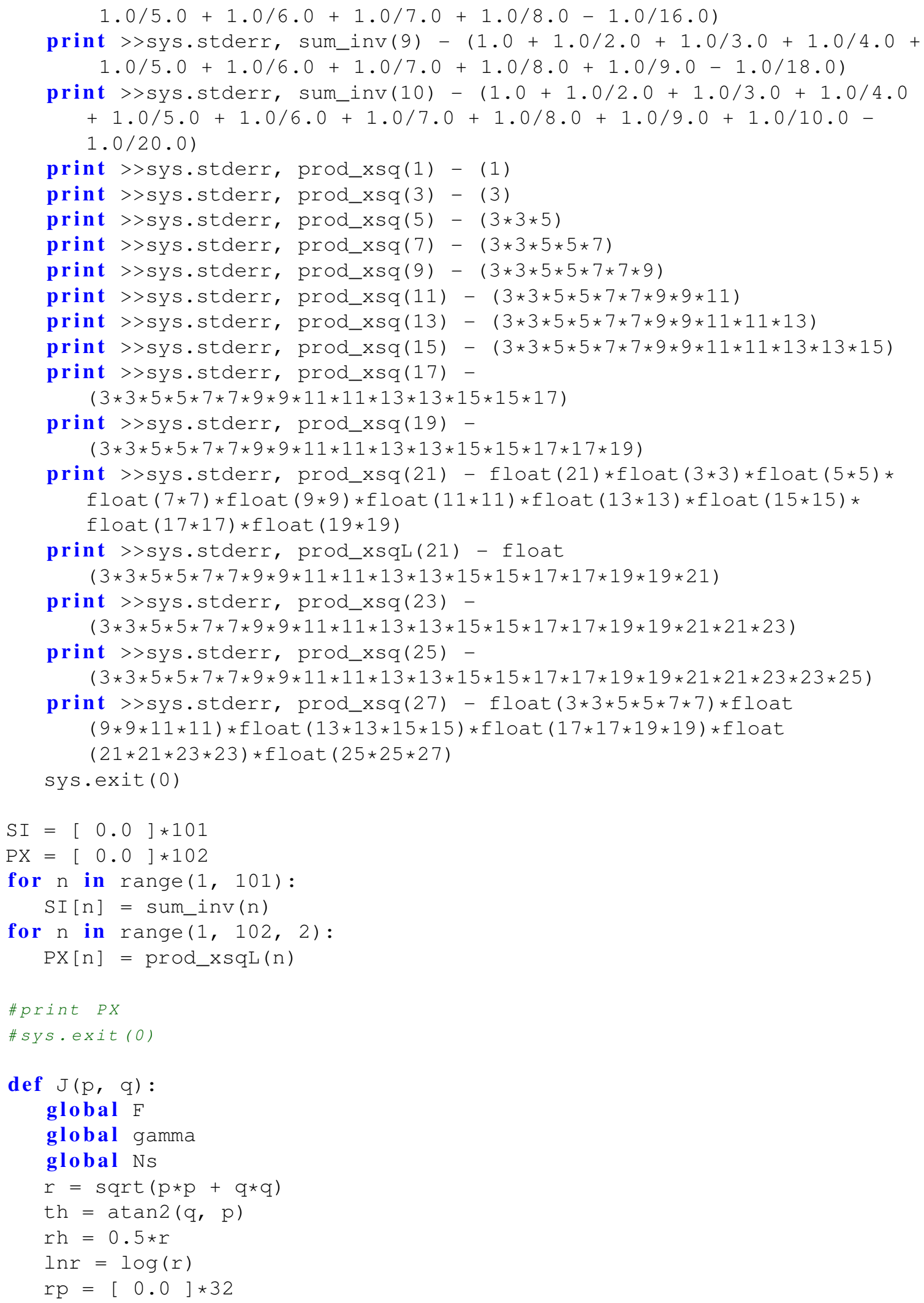




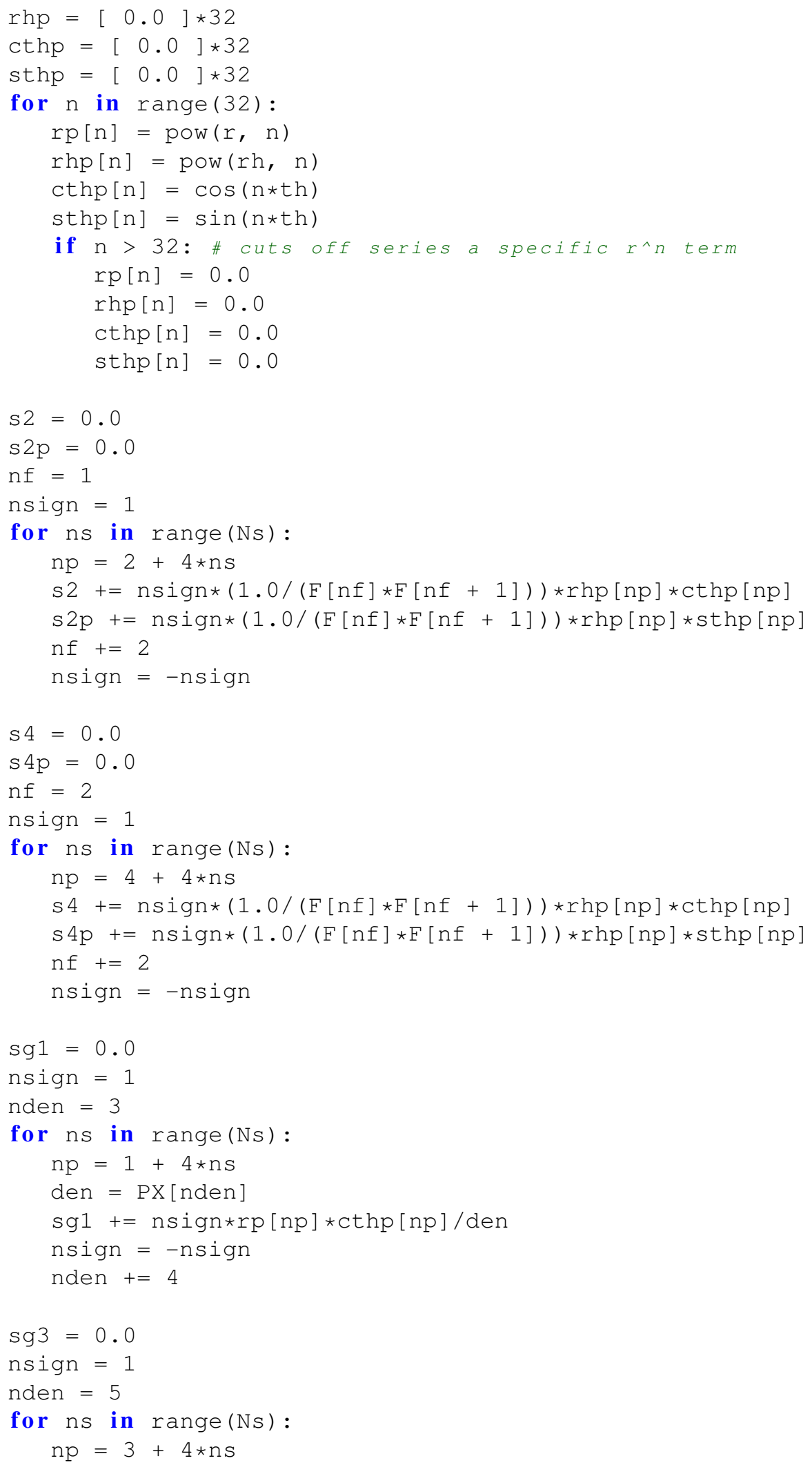




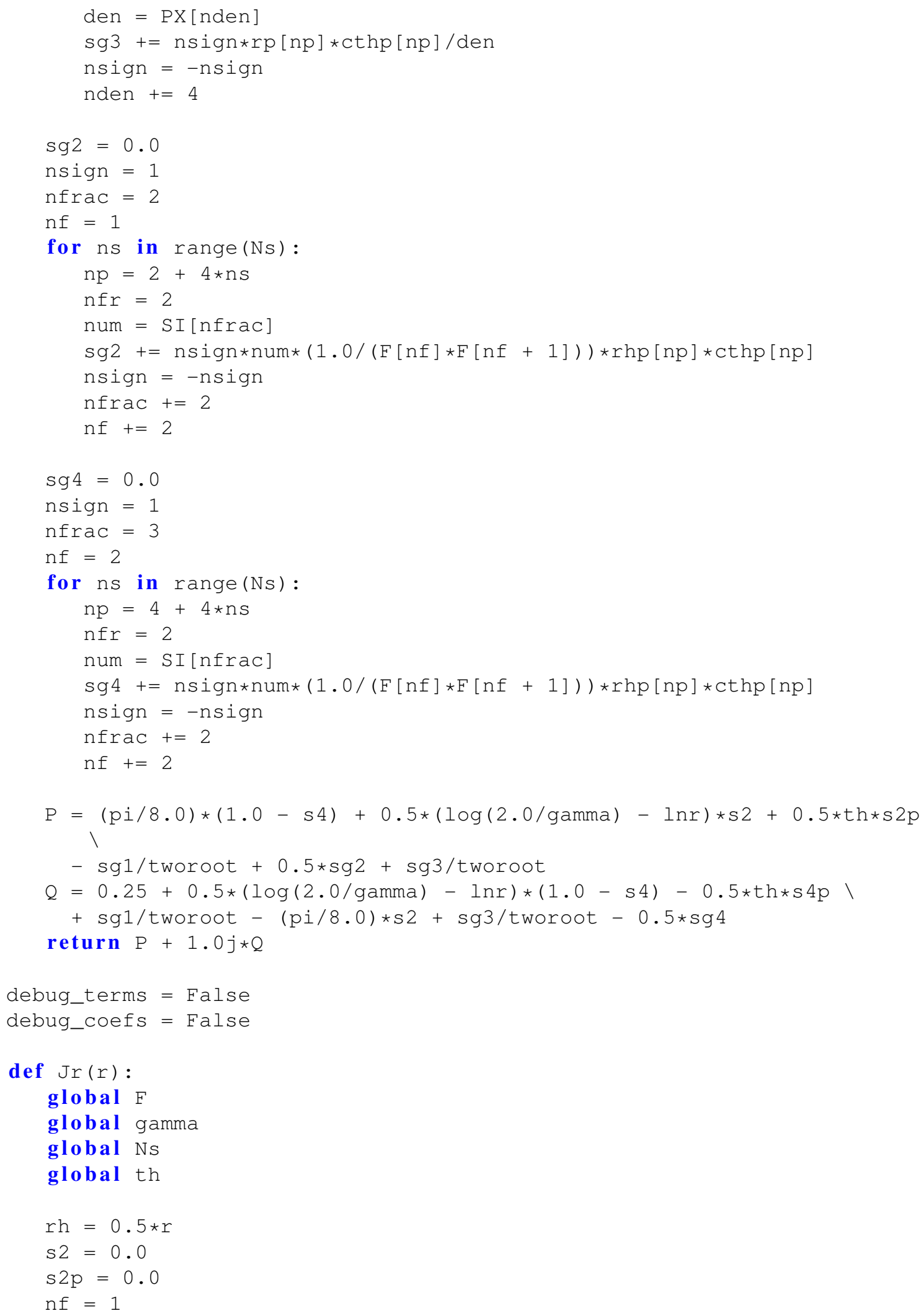




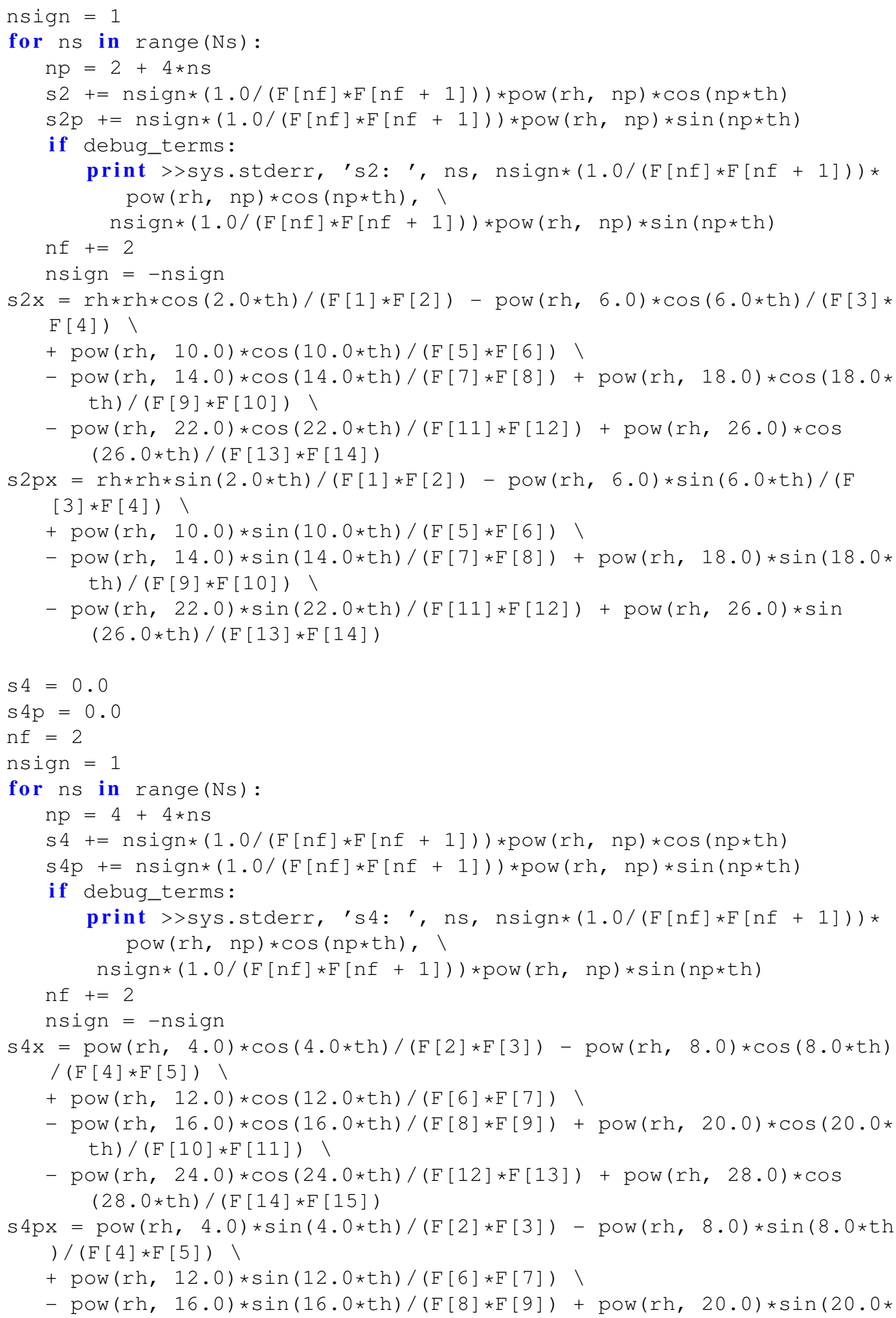


th) $/(\mathrm{F}[10] * \mathrm{~F}[11]) \backslash$

$-\operatorname{pow}(r h, 24.0) * \sin (24.0 * t h) /(F[12] \star F[13])+\operatorname{pow}(r h, 28.0) \star \sin$

$(28.0 * t h) /(F[14] * F[15])$

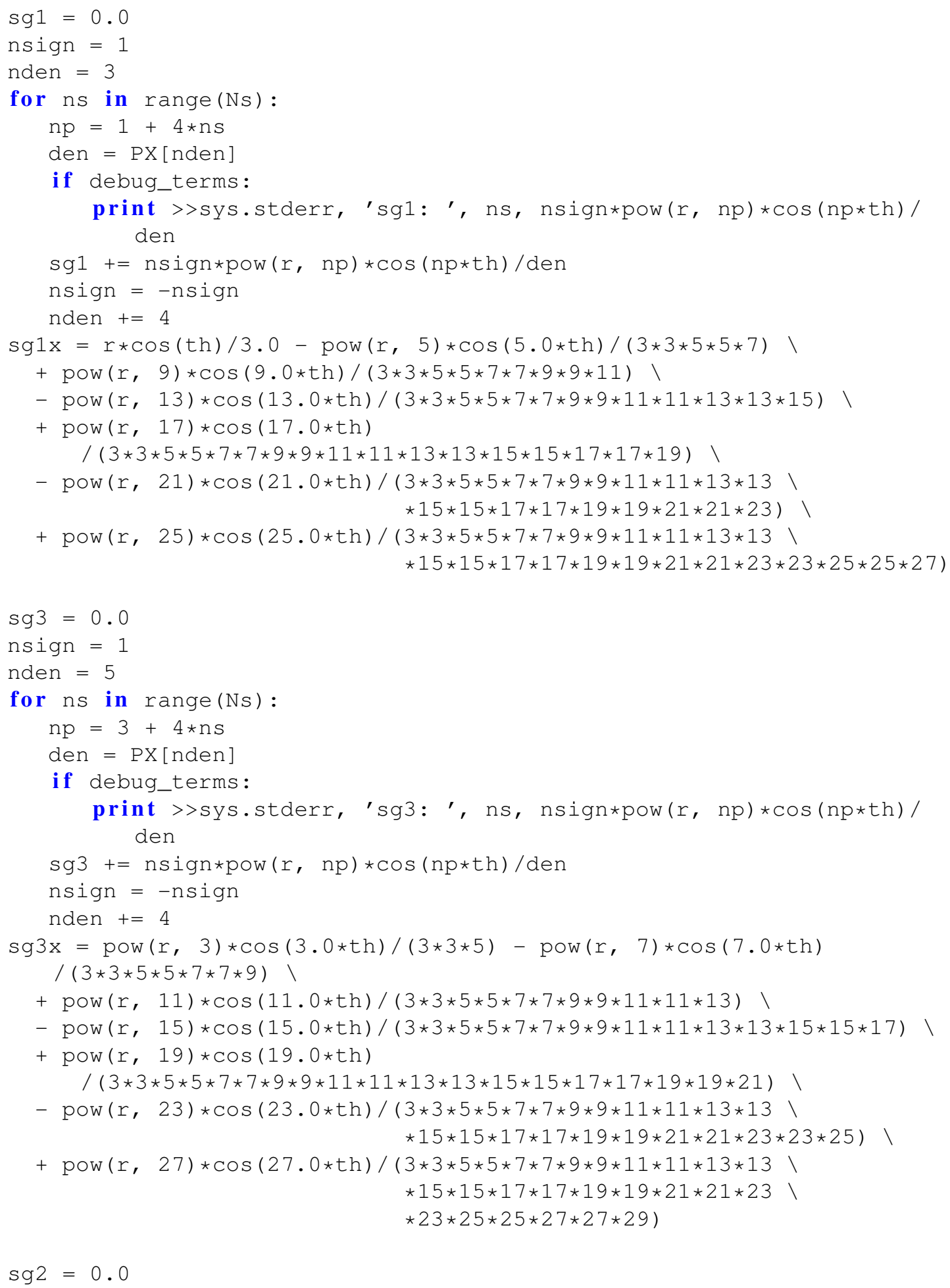




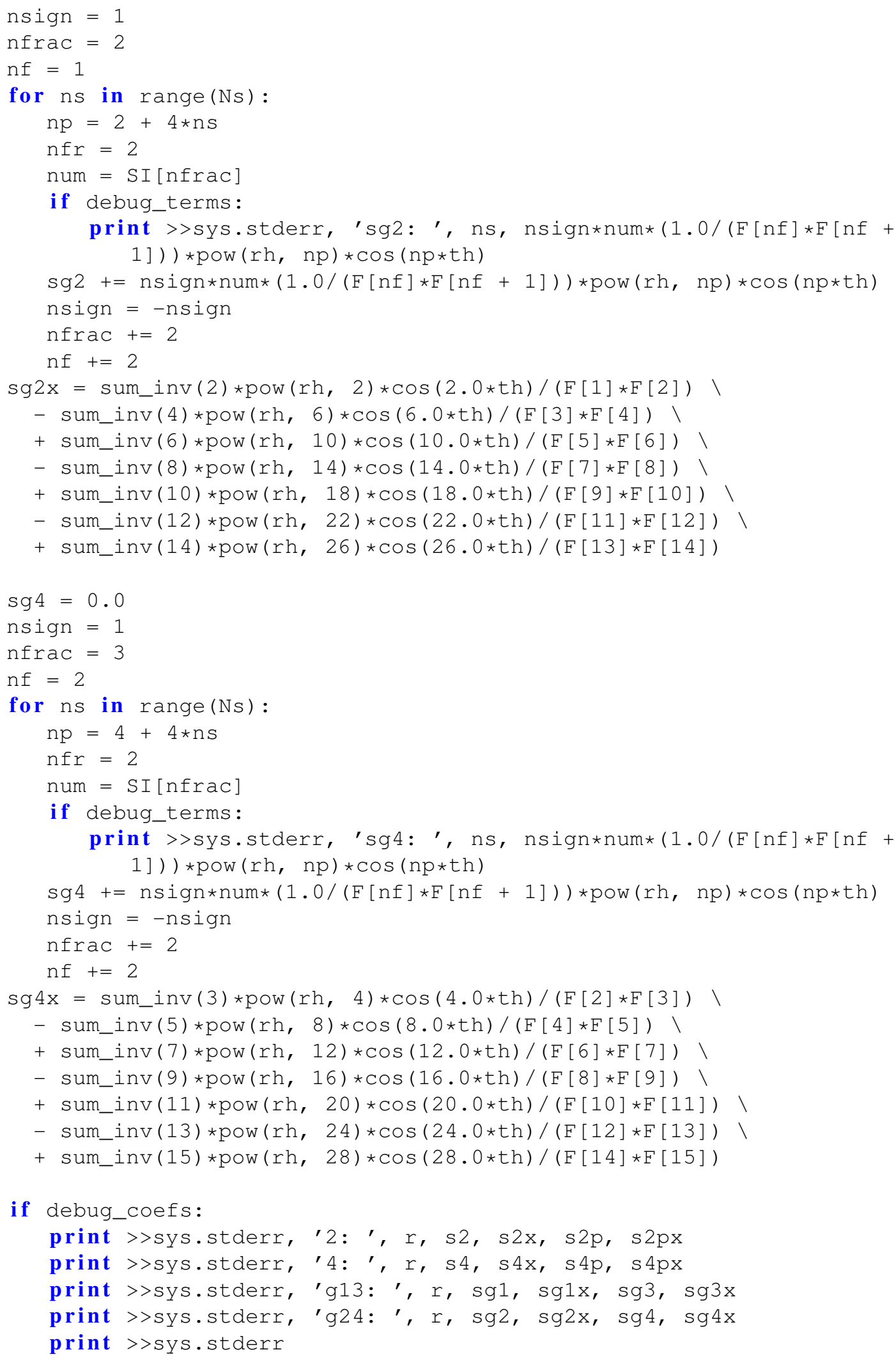




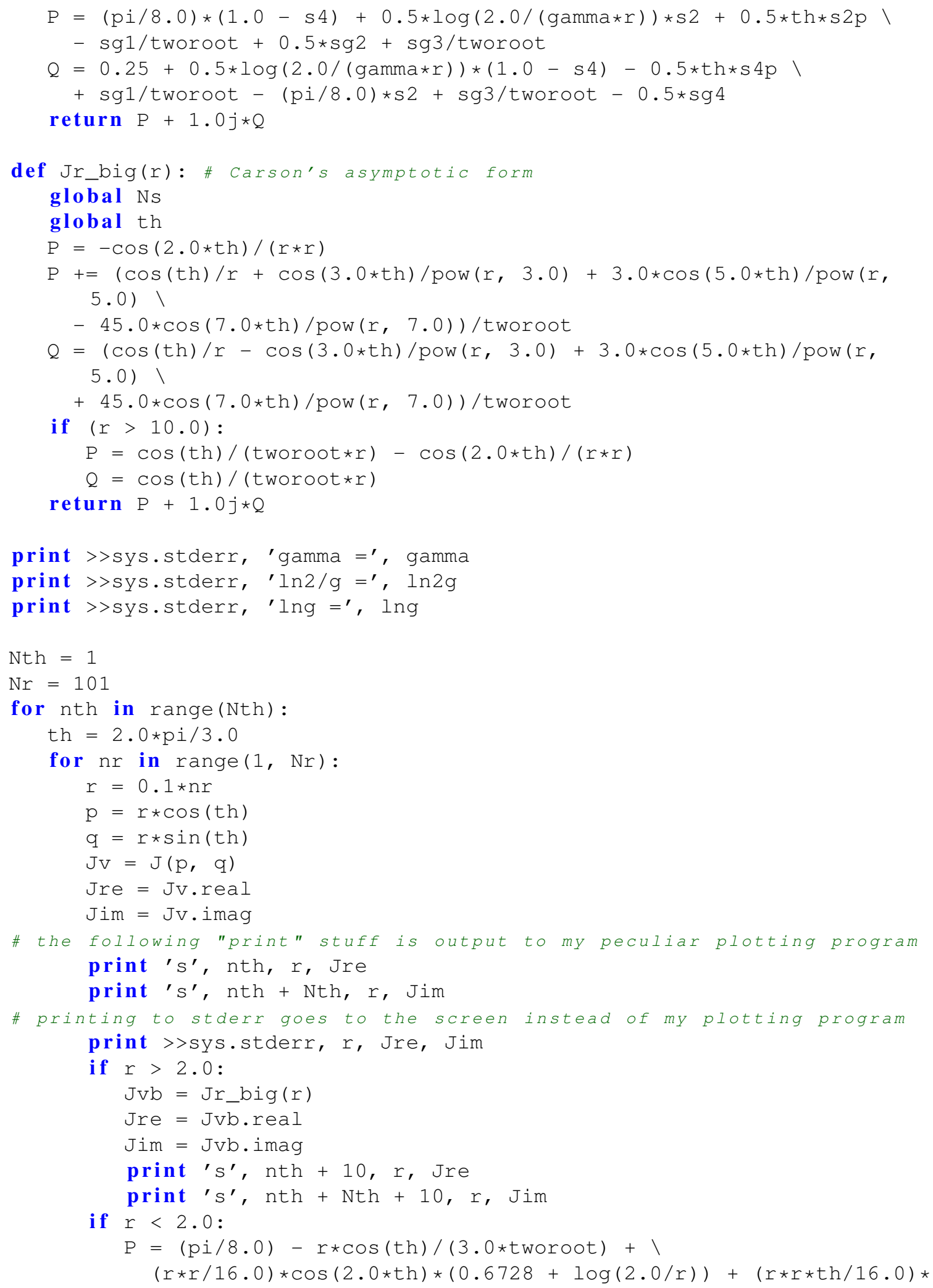




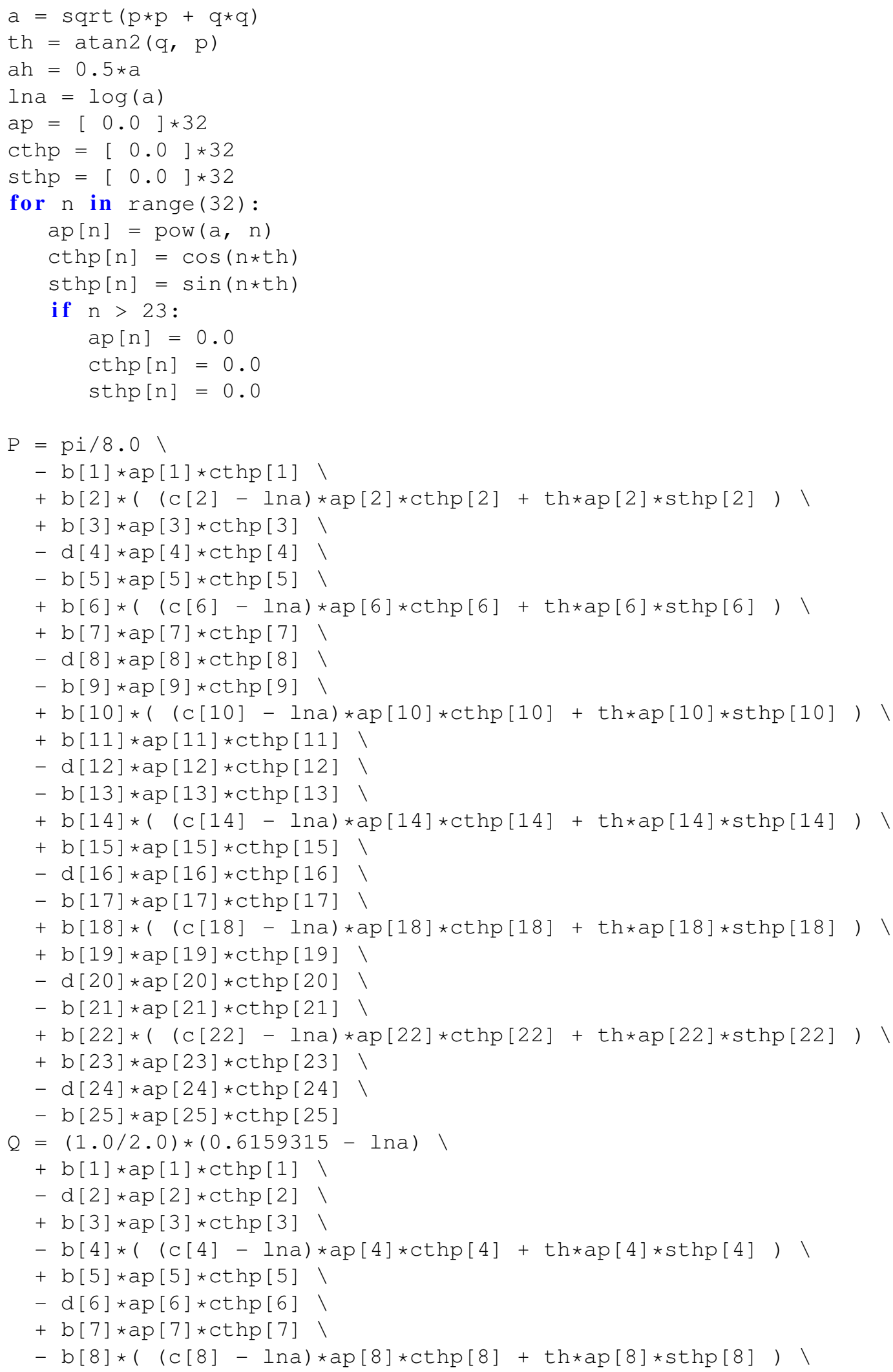




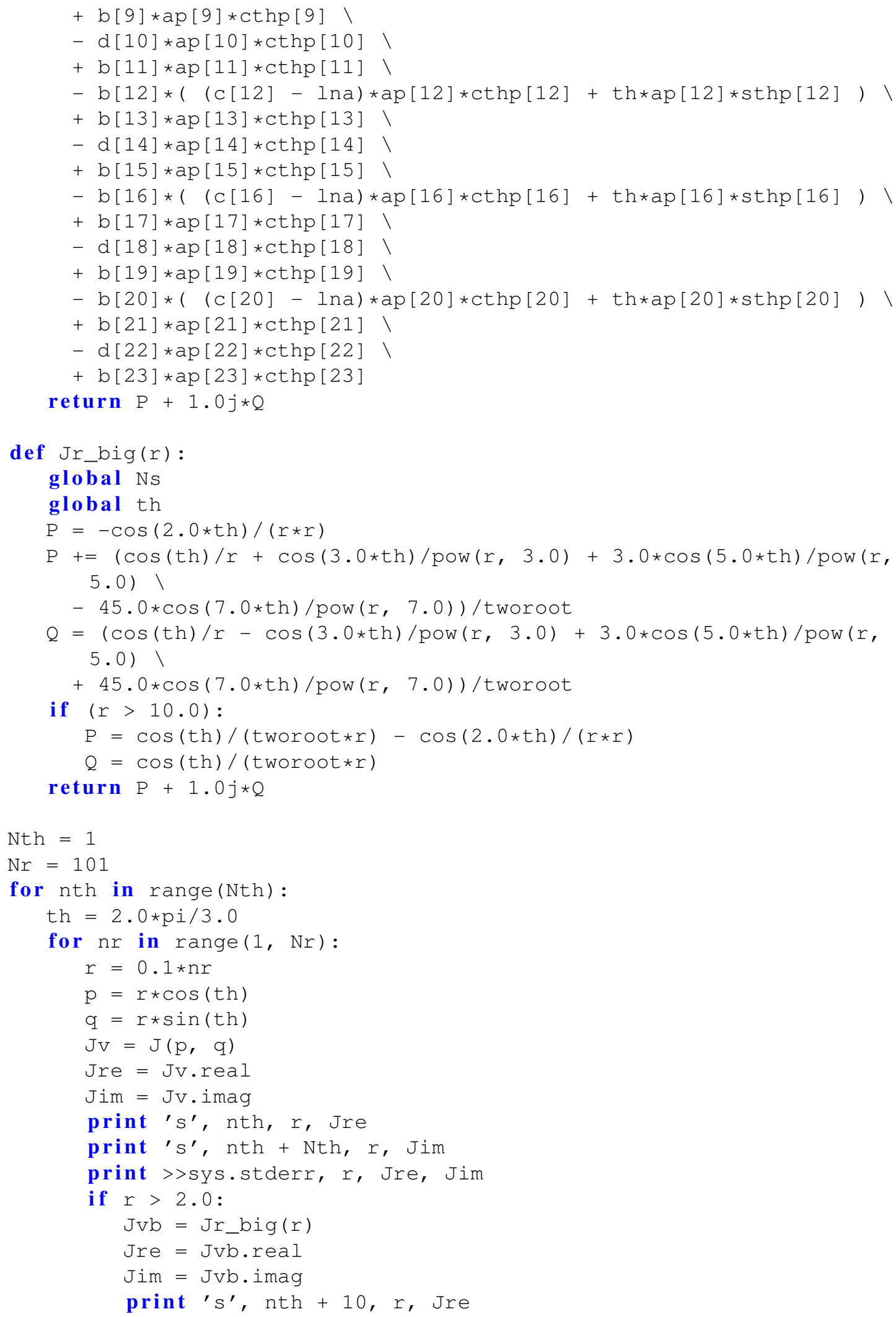




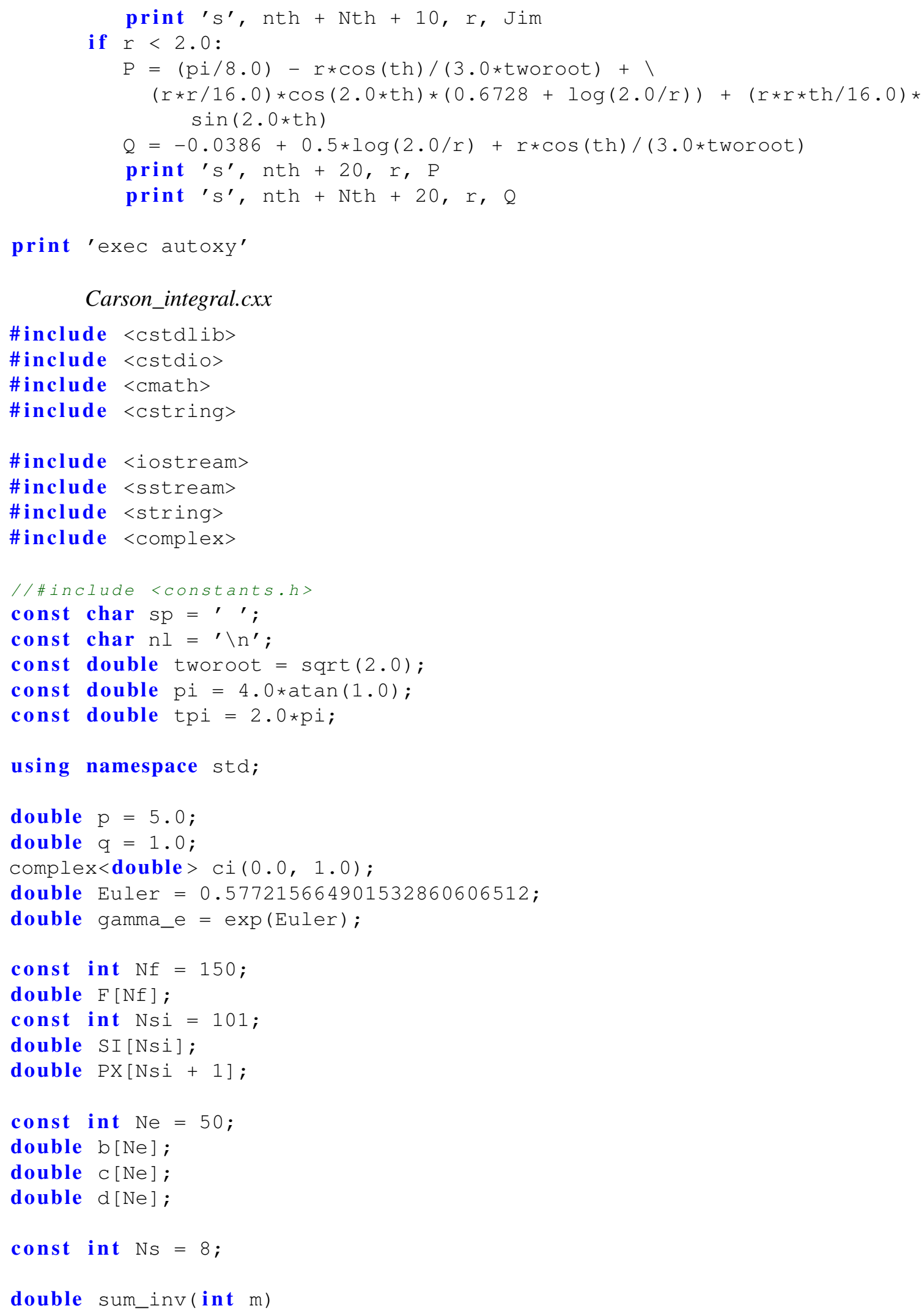




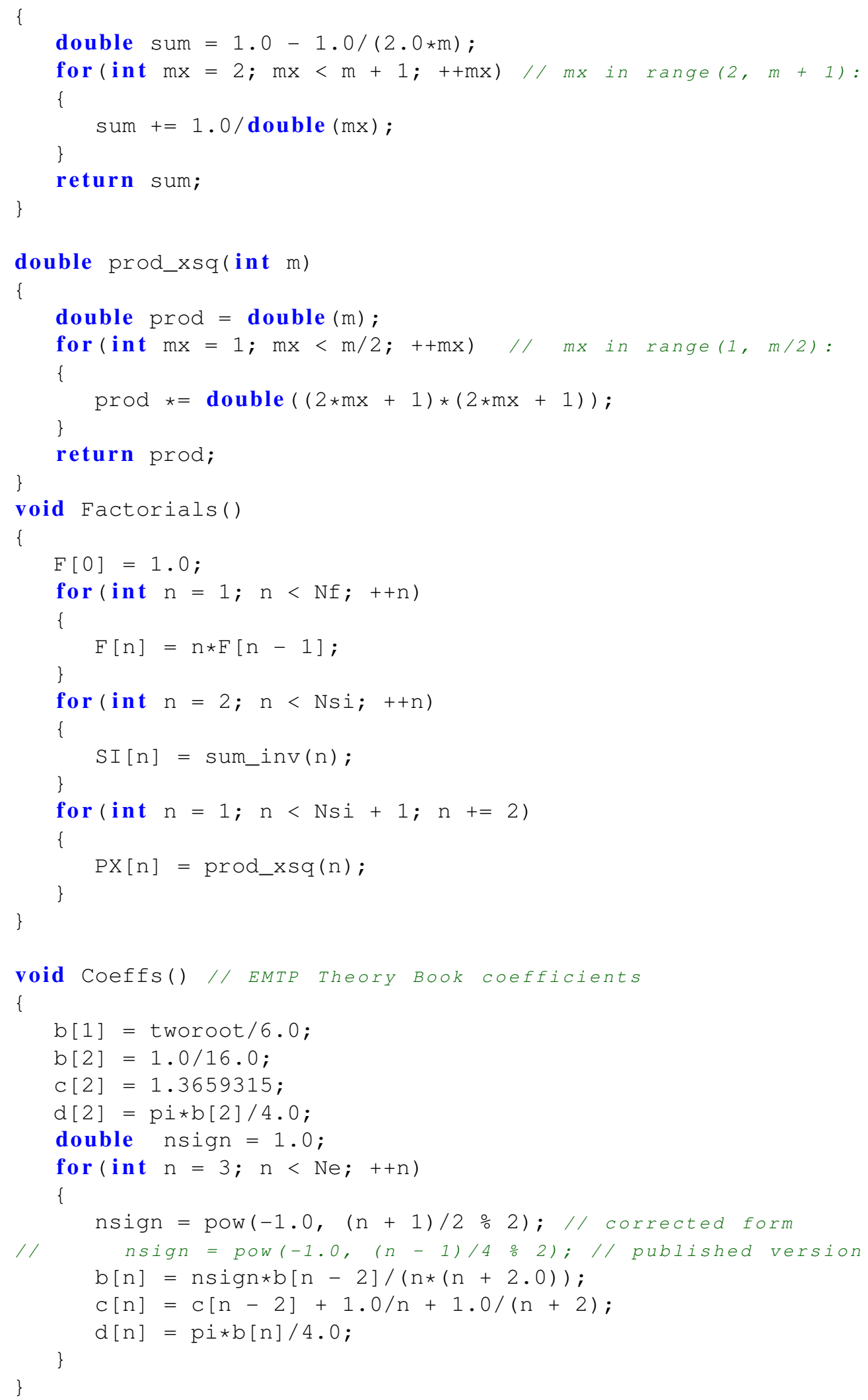




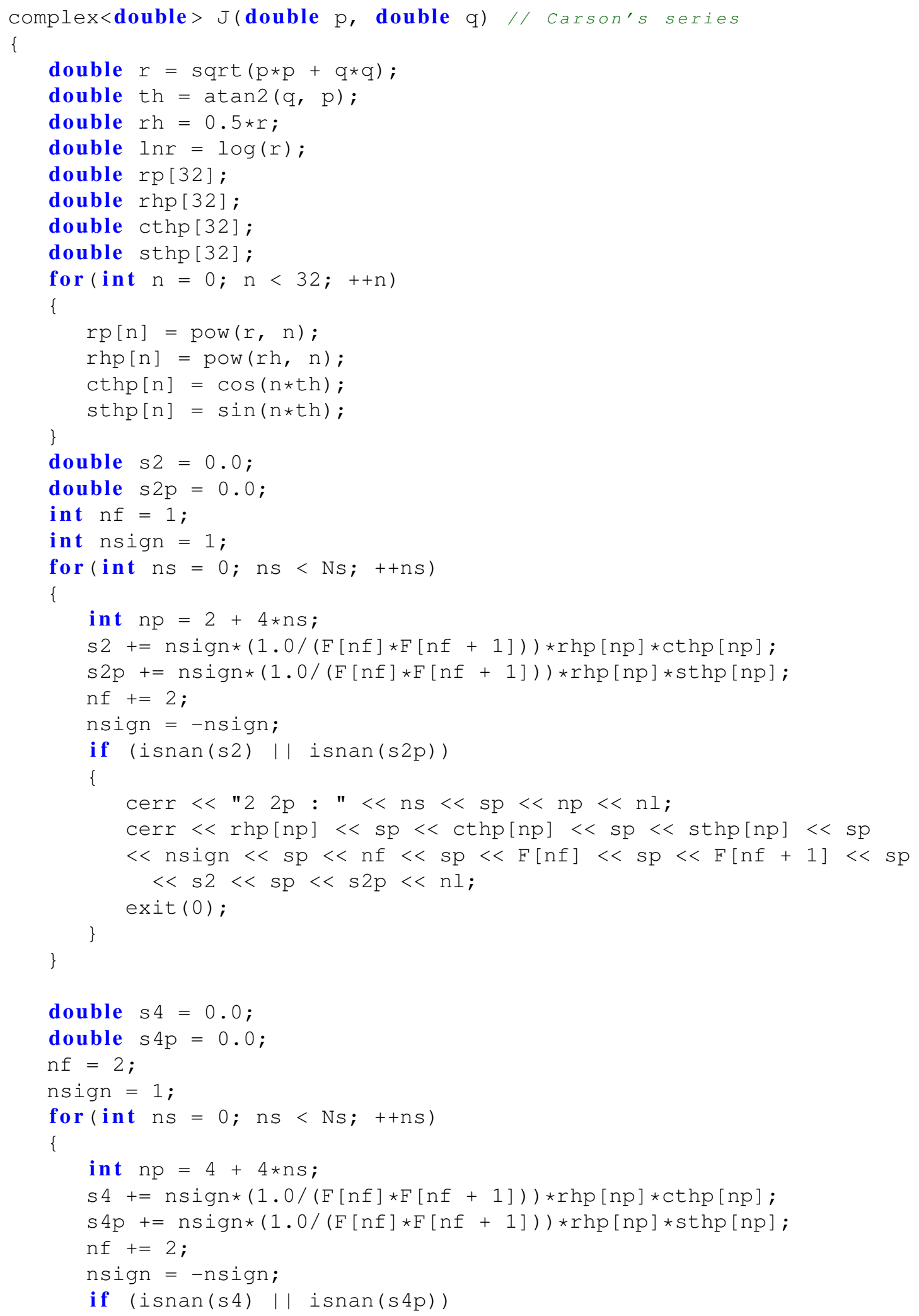




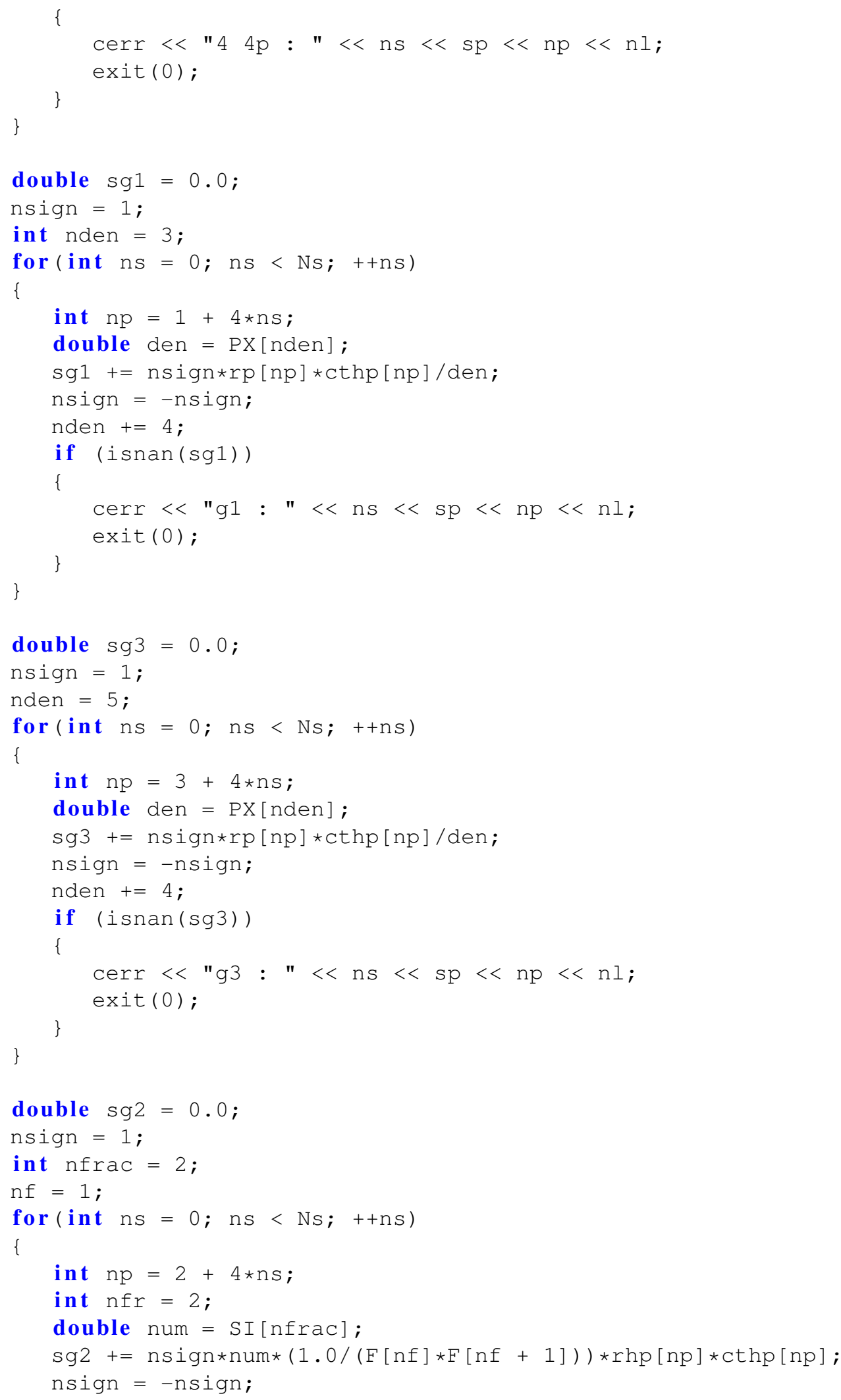




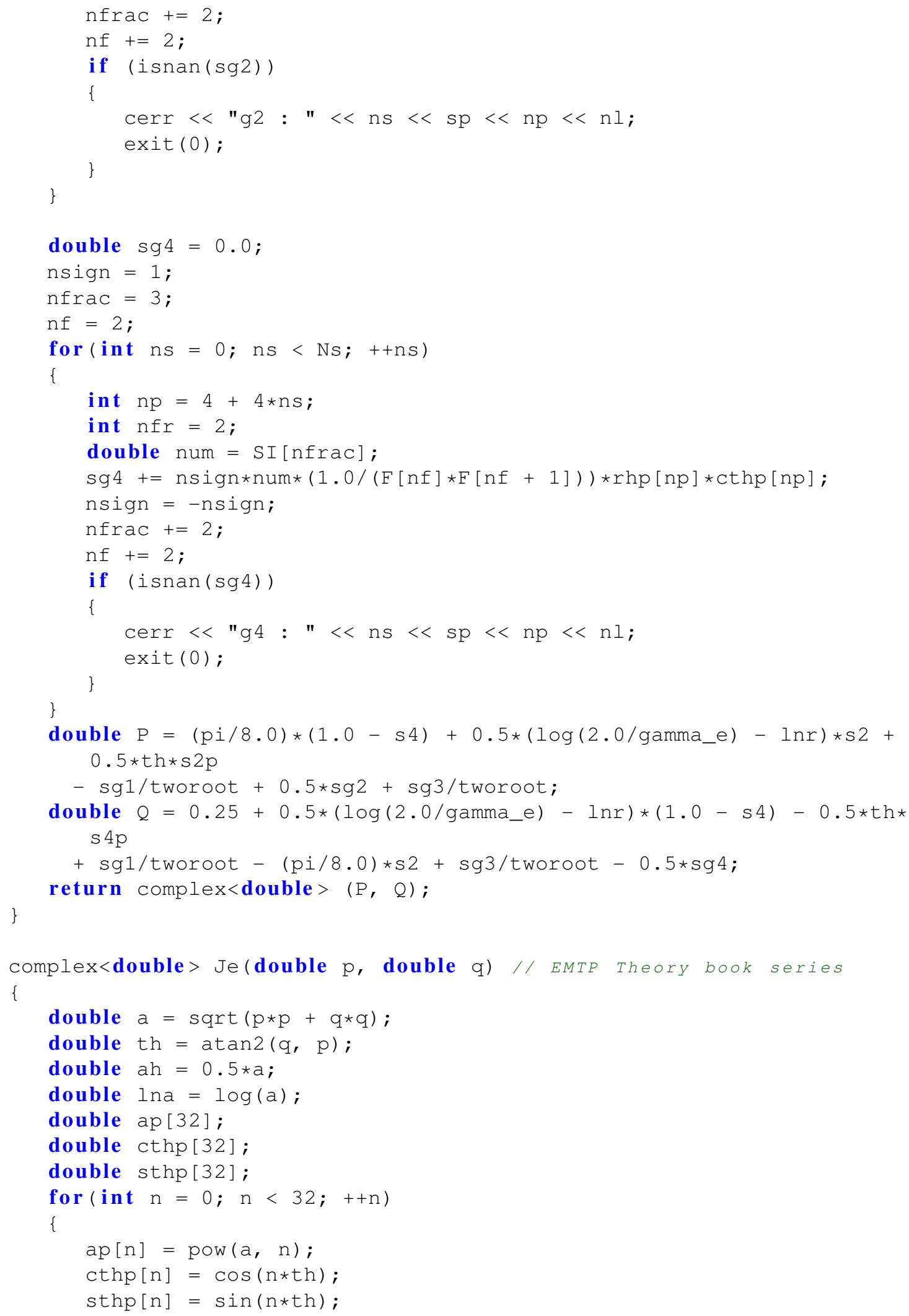




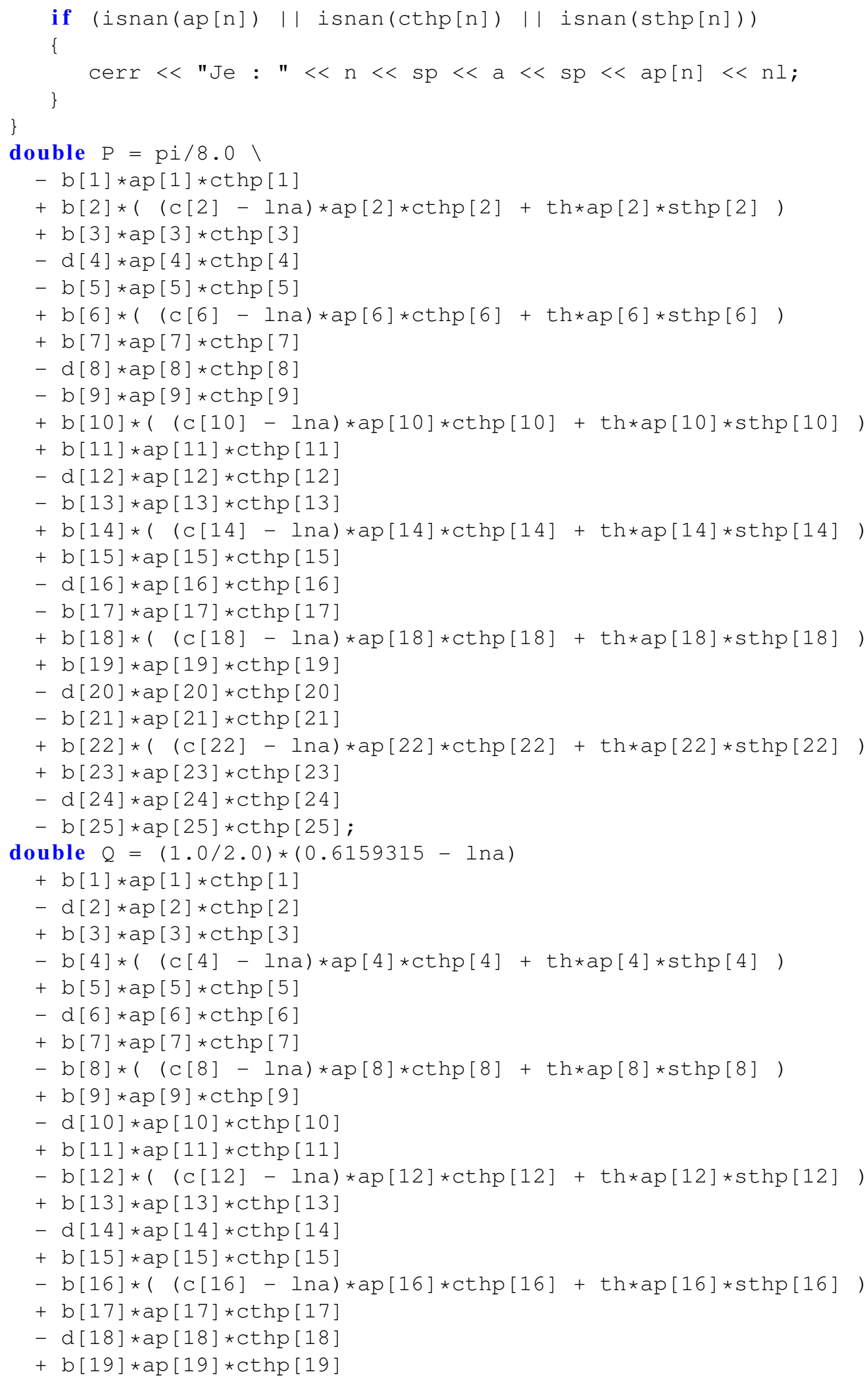




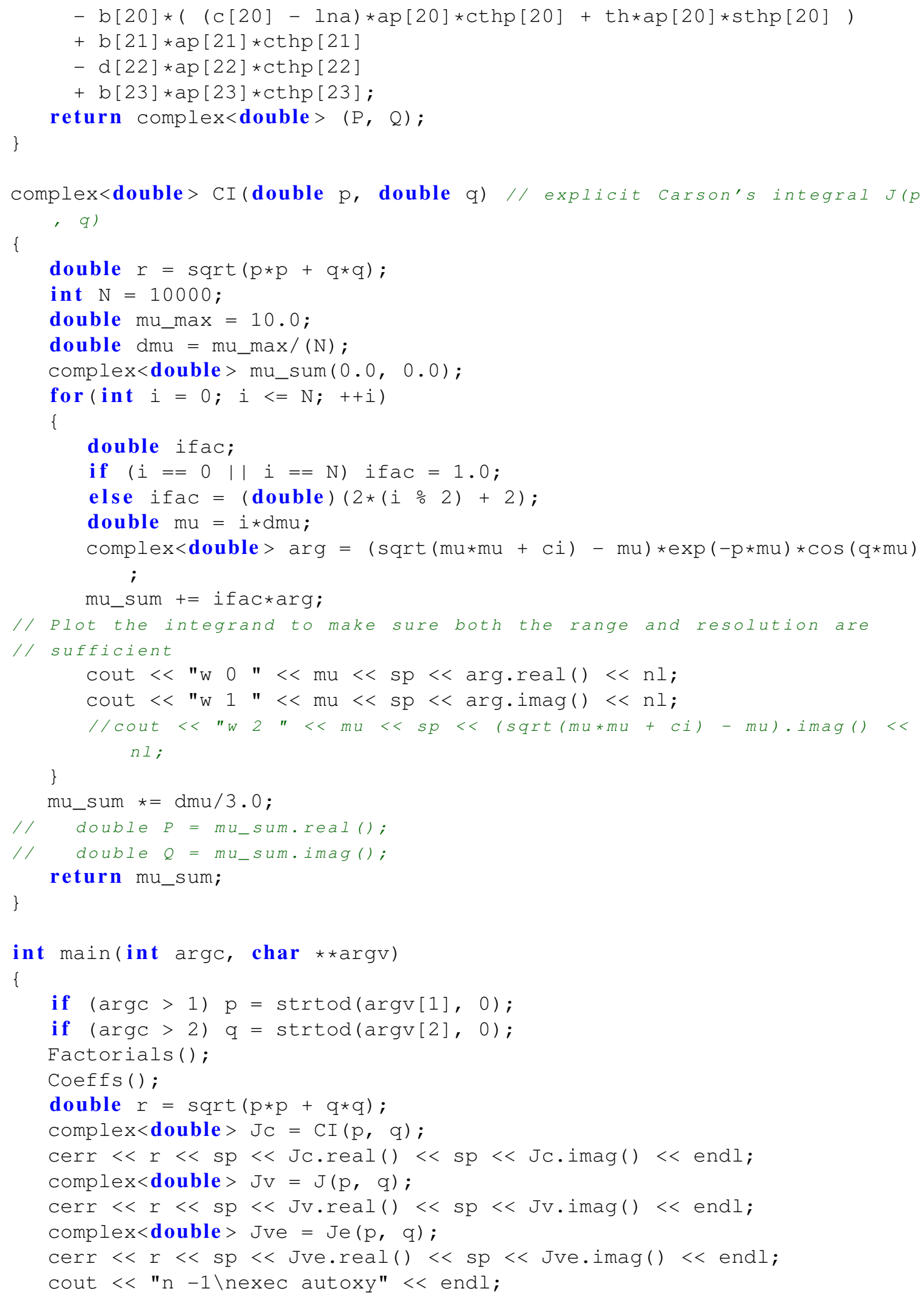


return 0 ;

\}

\section{A.2 EMTP Equations in Matlab}

\section{A.2.1 Carson's Formula}

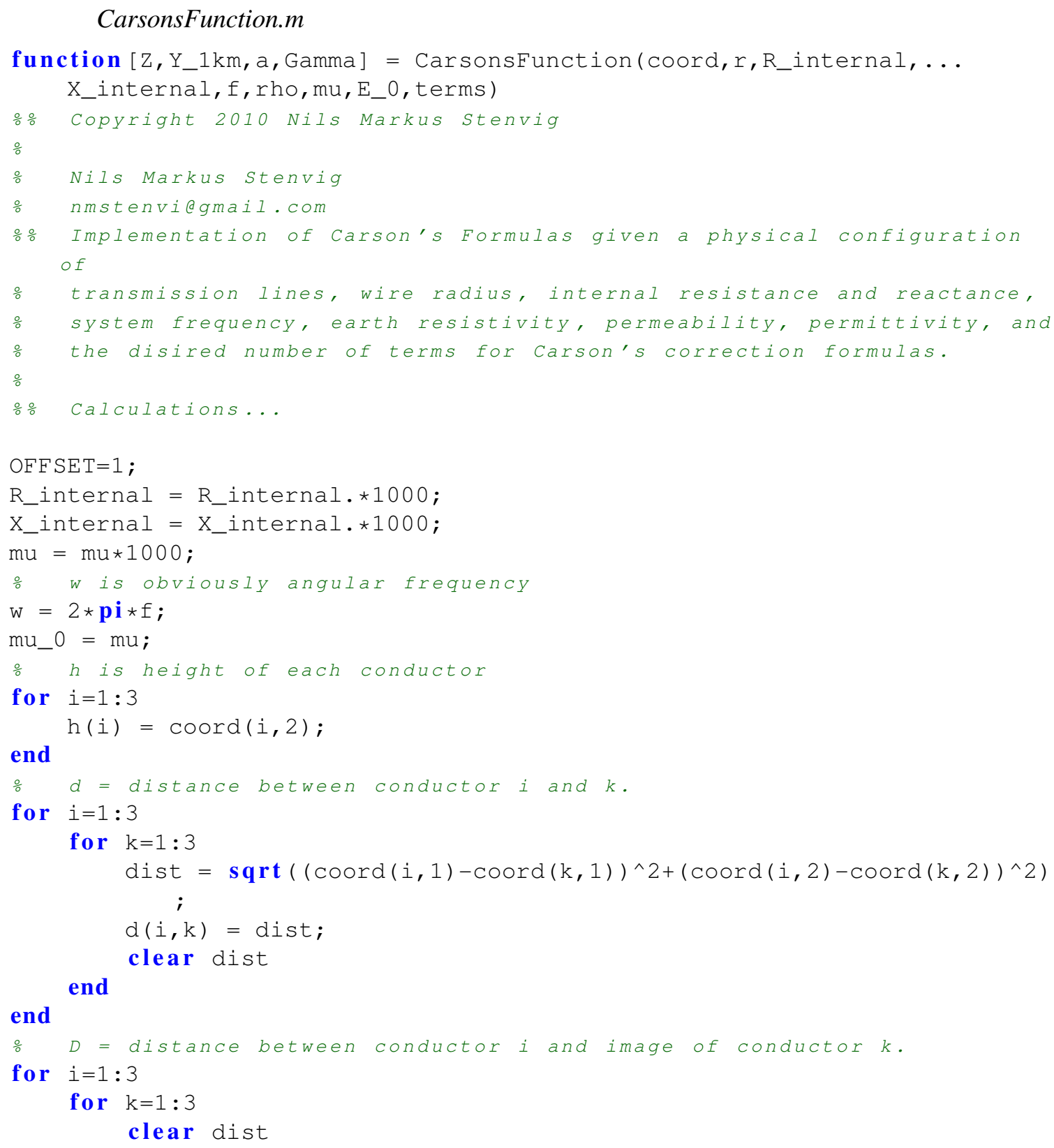




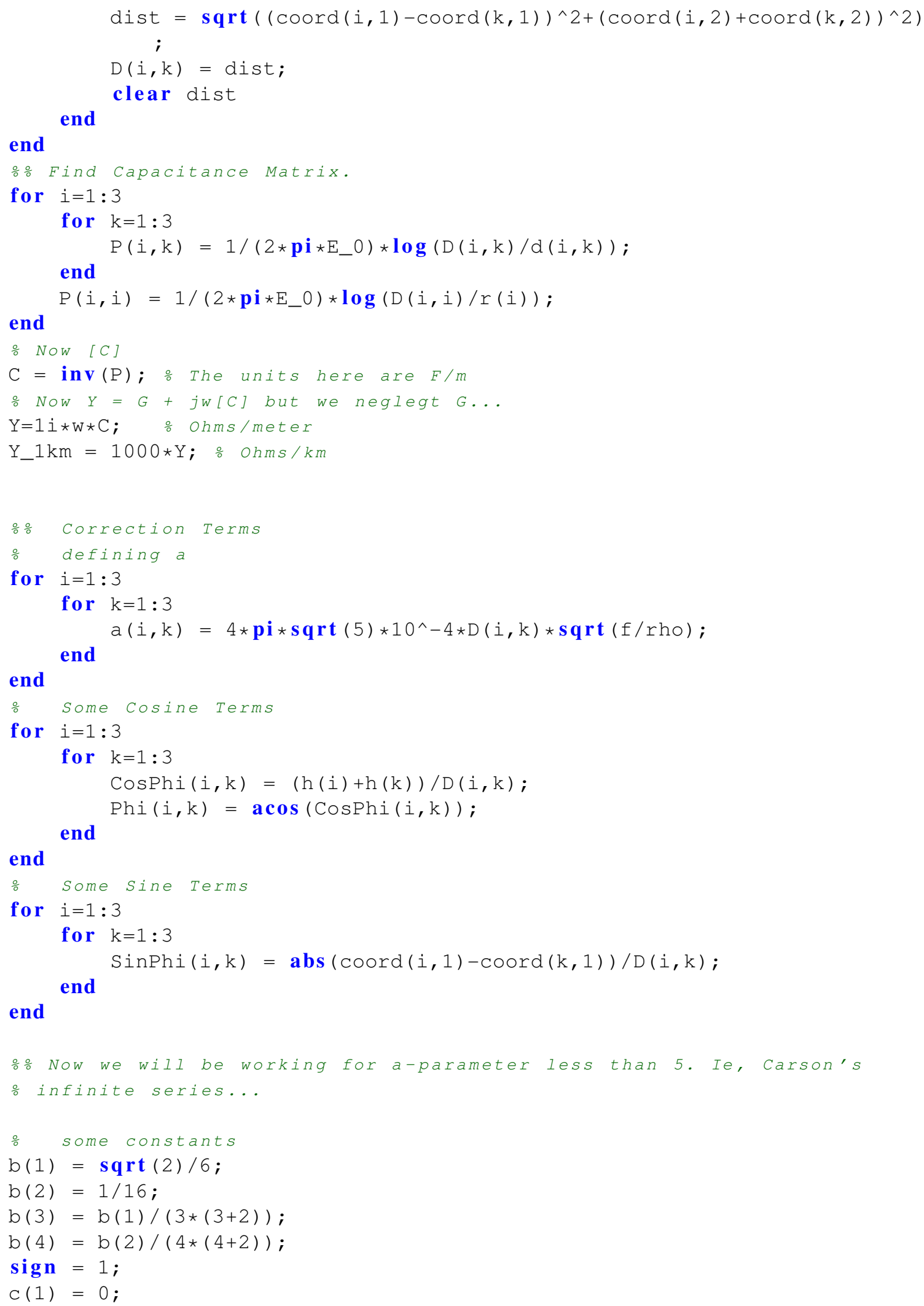




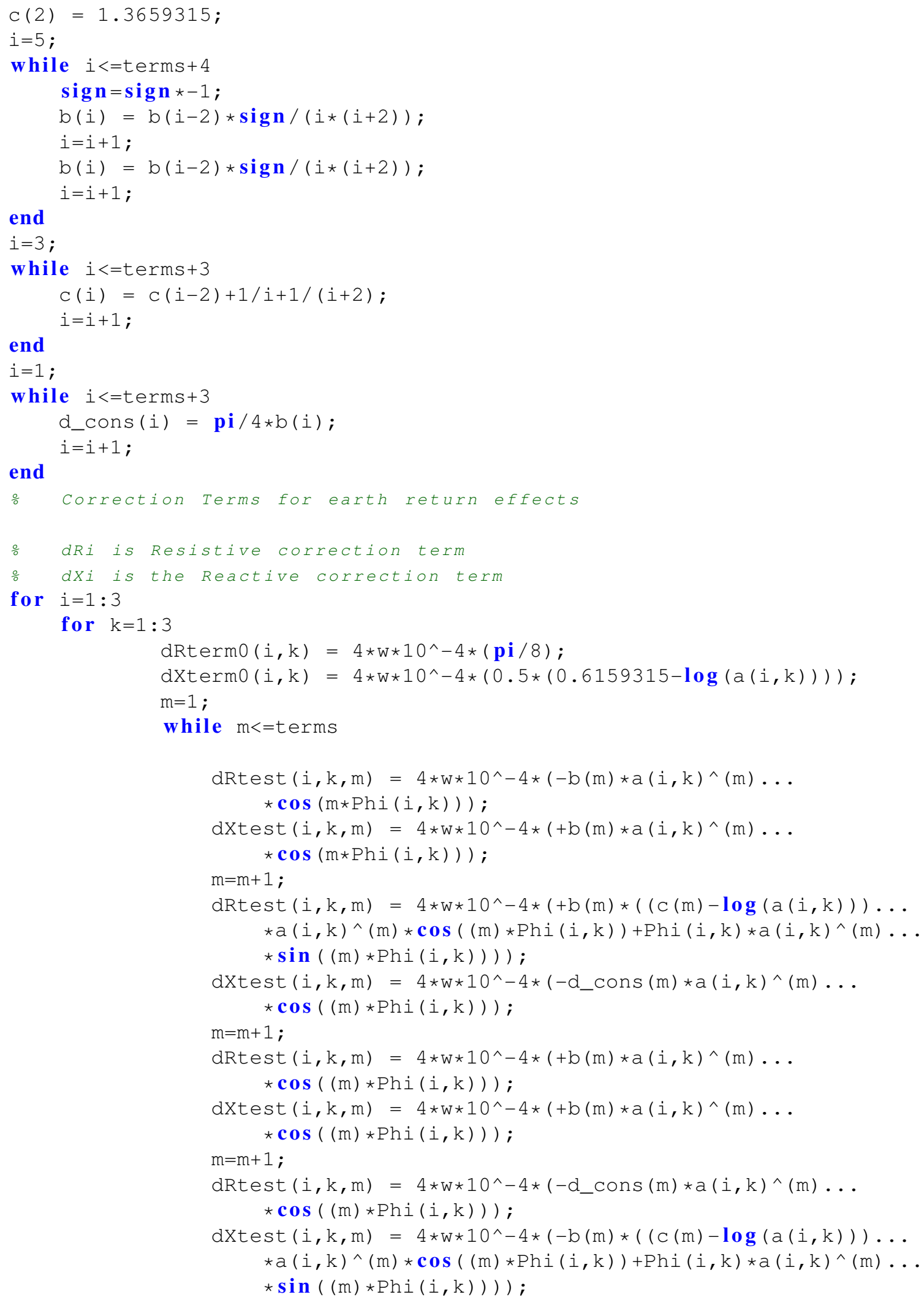




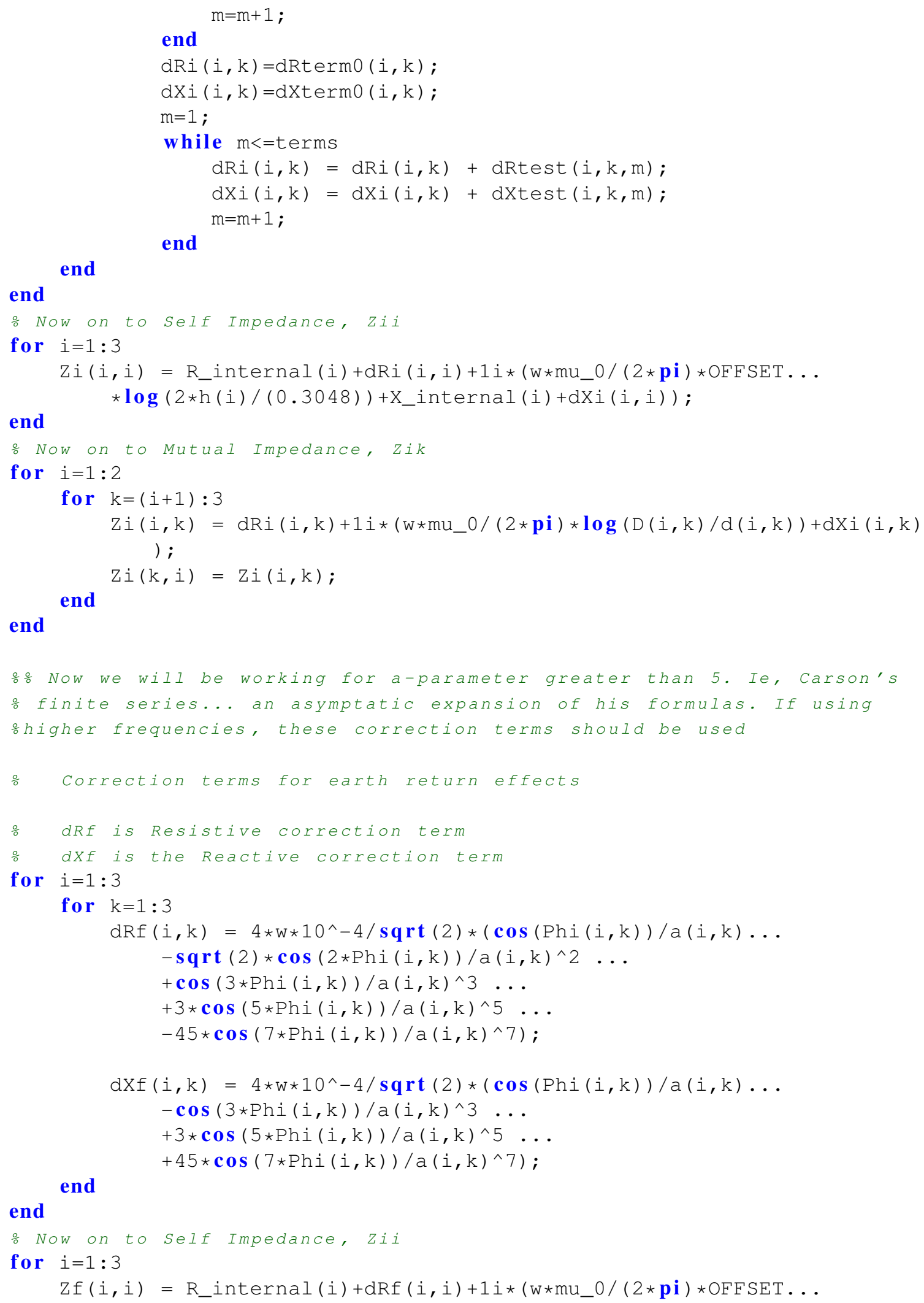




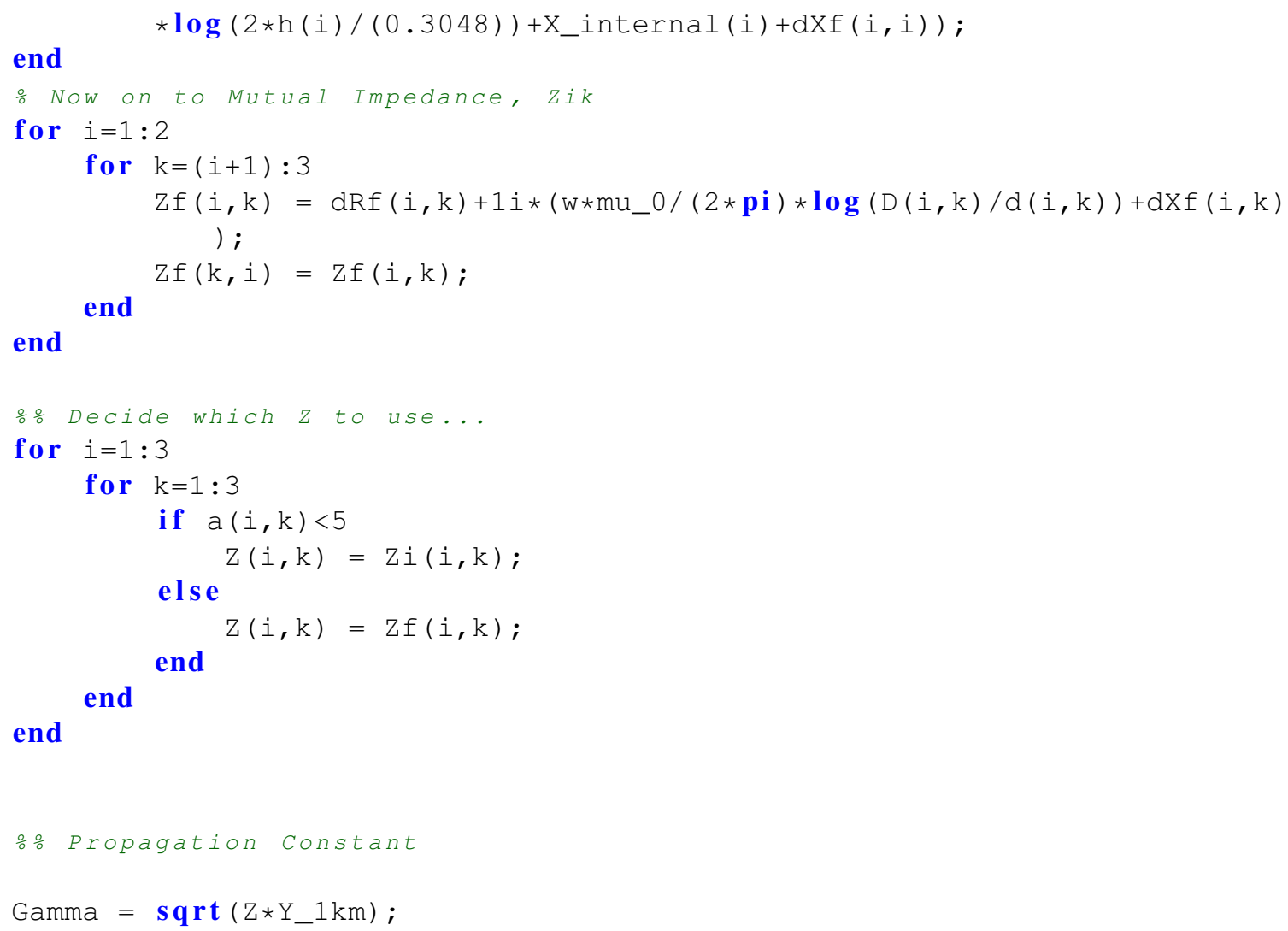

\section{A.2.2 Propagation Constant Calculation}

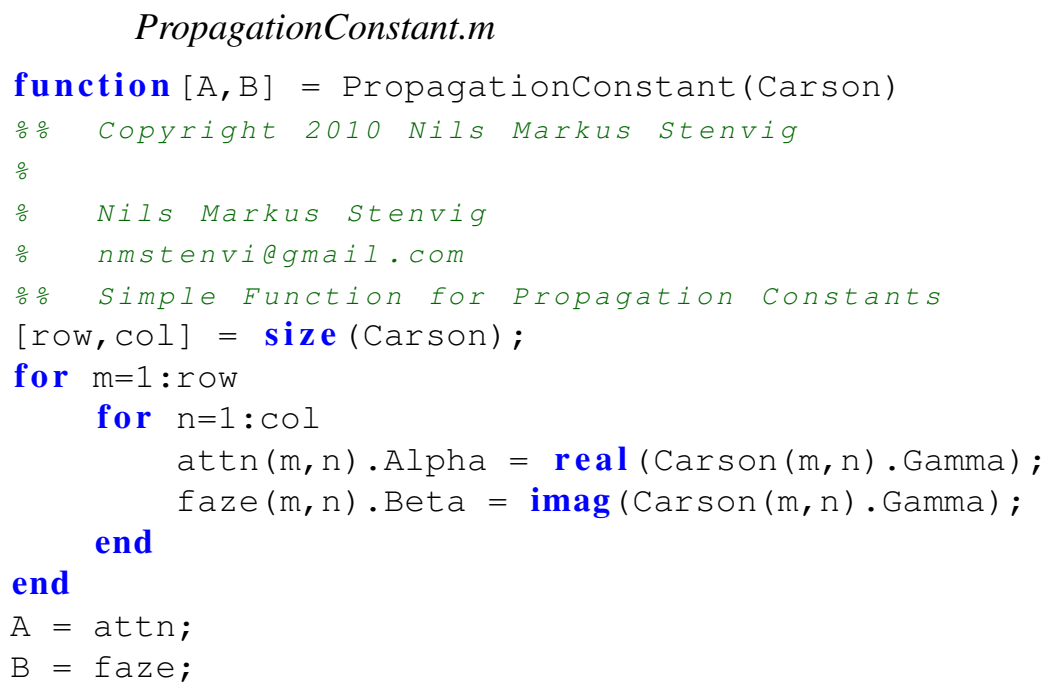

\section{A.2.3 3 Conductor Carson Example}




\section{LogCarsons.m}

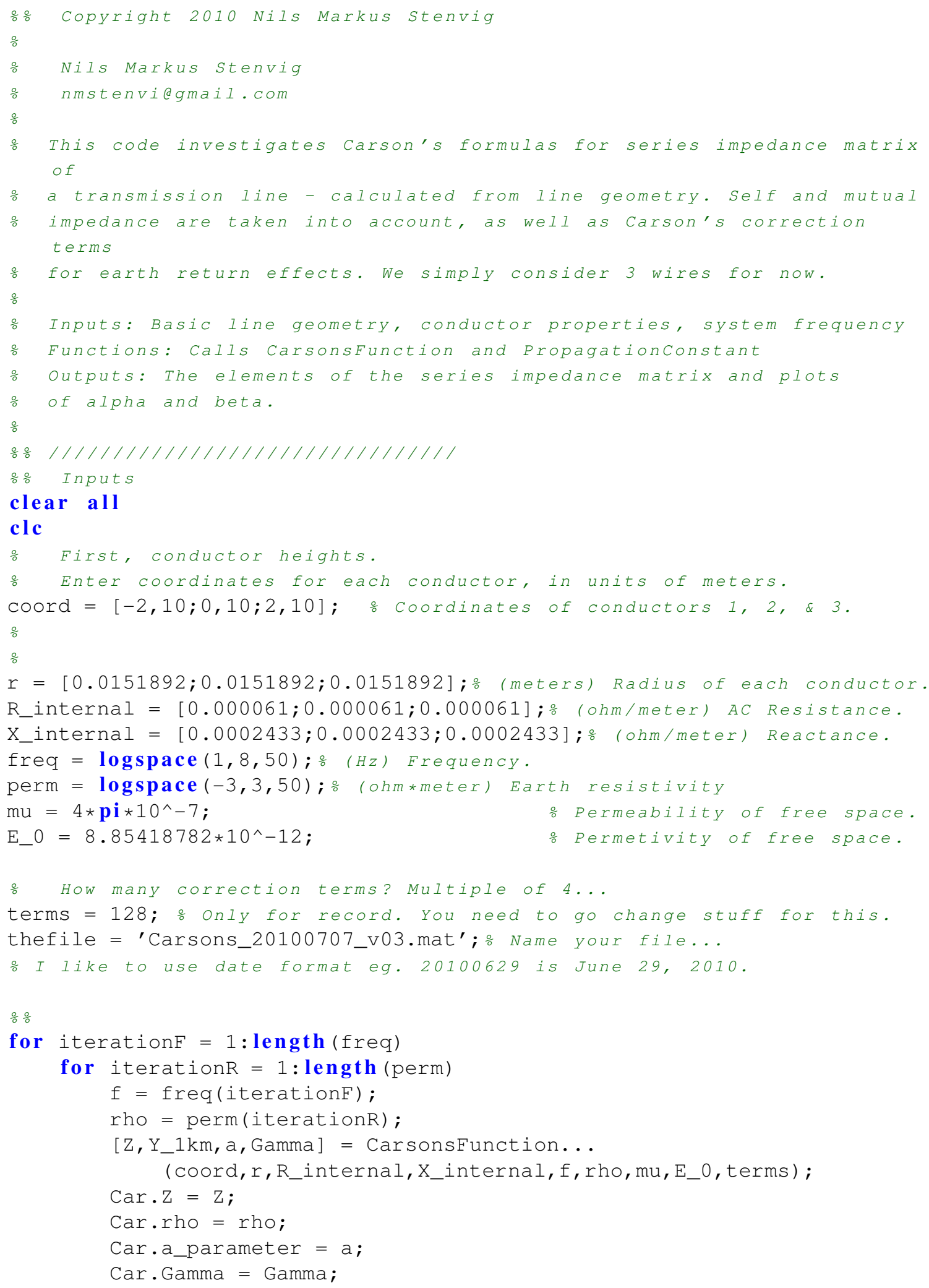




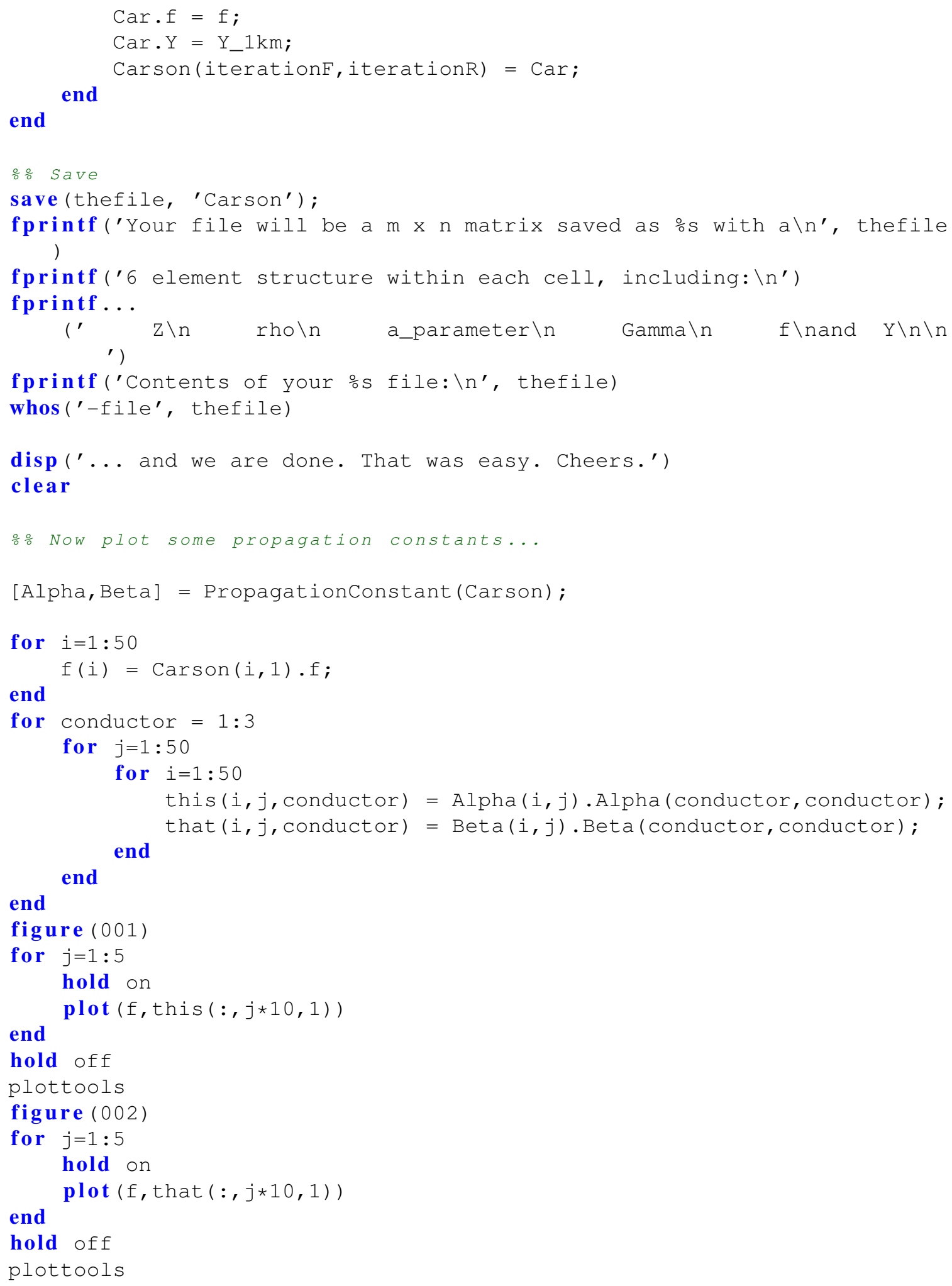




\section{A.3 ATP Propagation Constants in Matlab}

\section{A.3.1 Reading ATP .lis File}

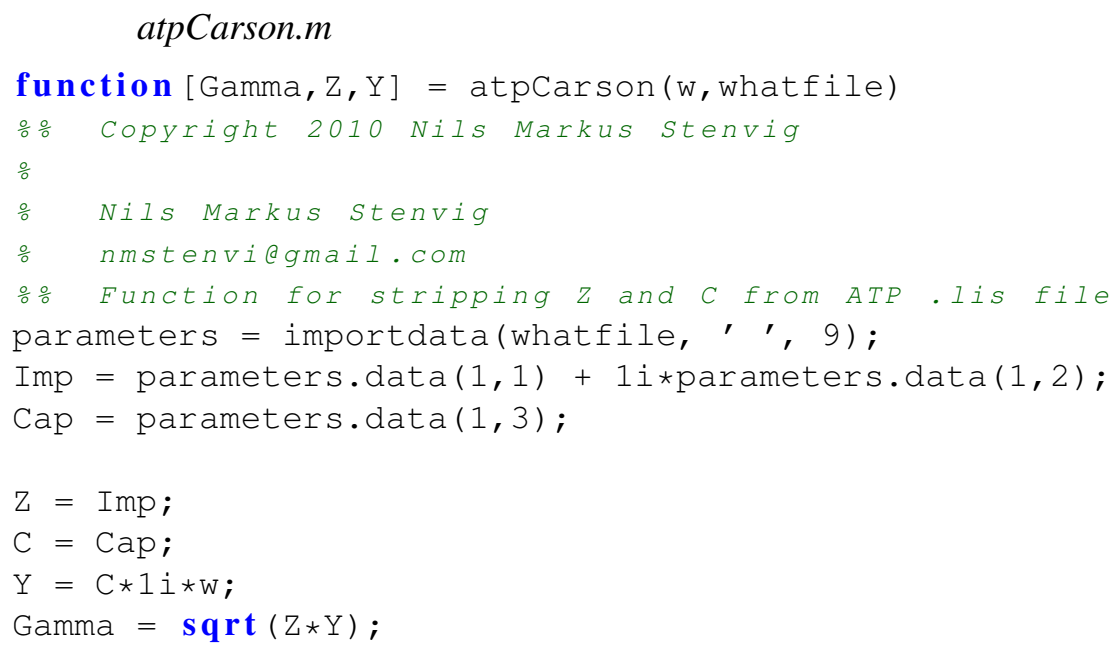

\section{A.3.2 Calculating Propagation Constants}

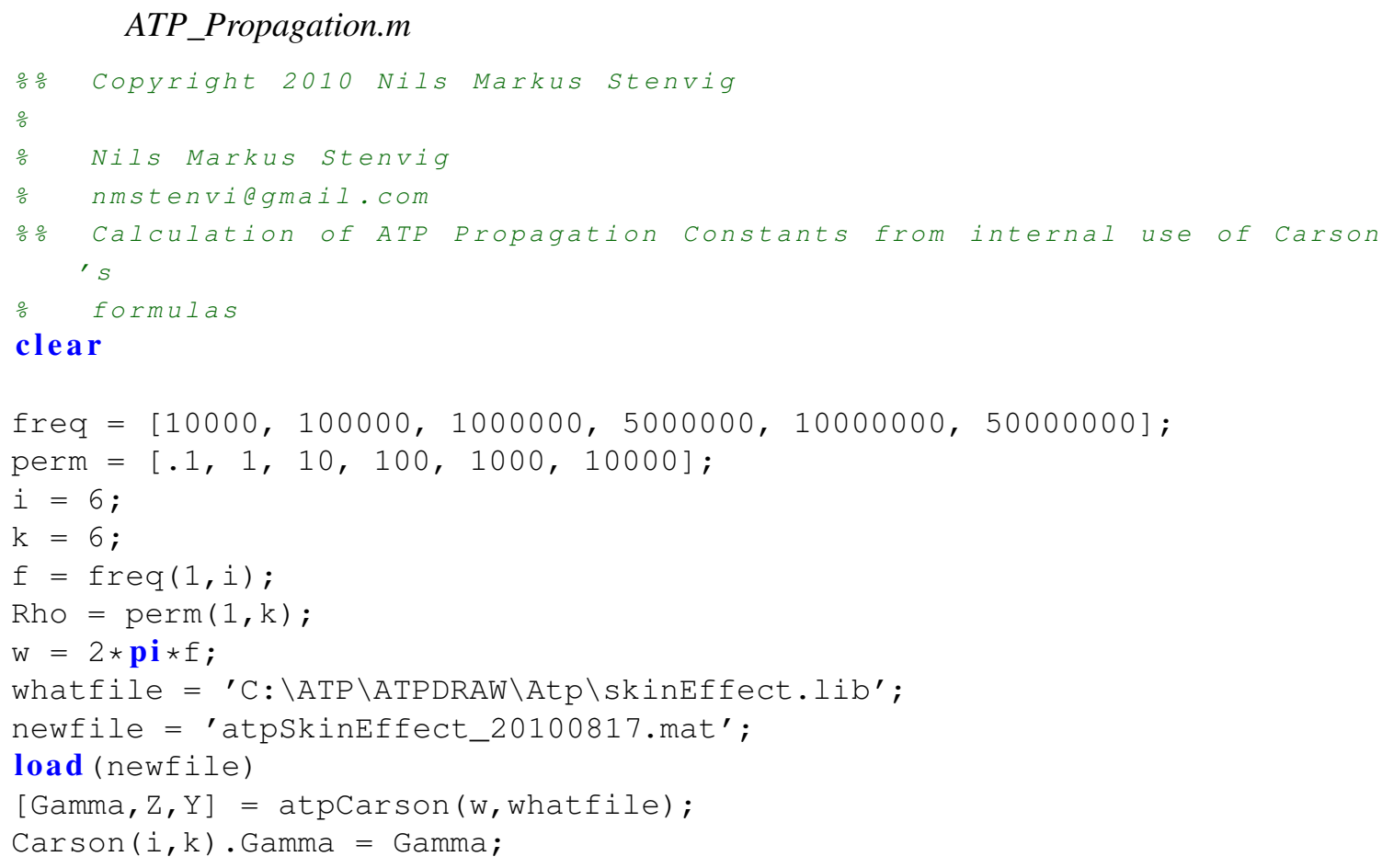




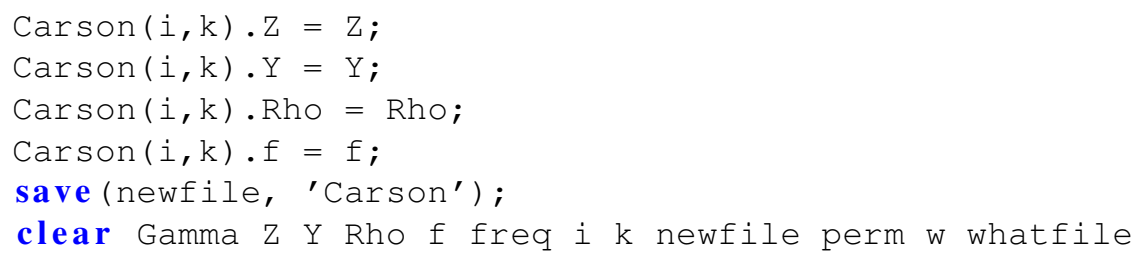

\section{A.3.3 Plotting Example Code}

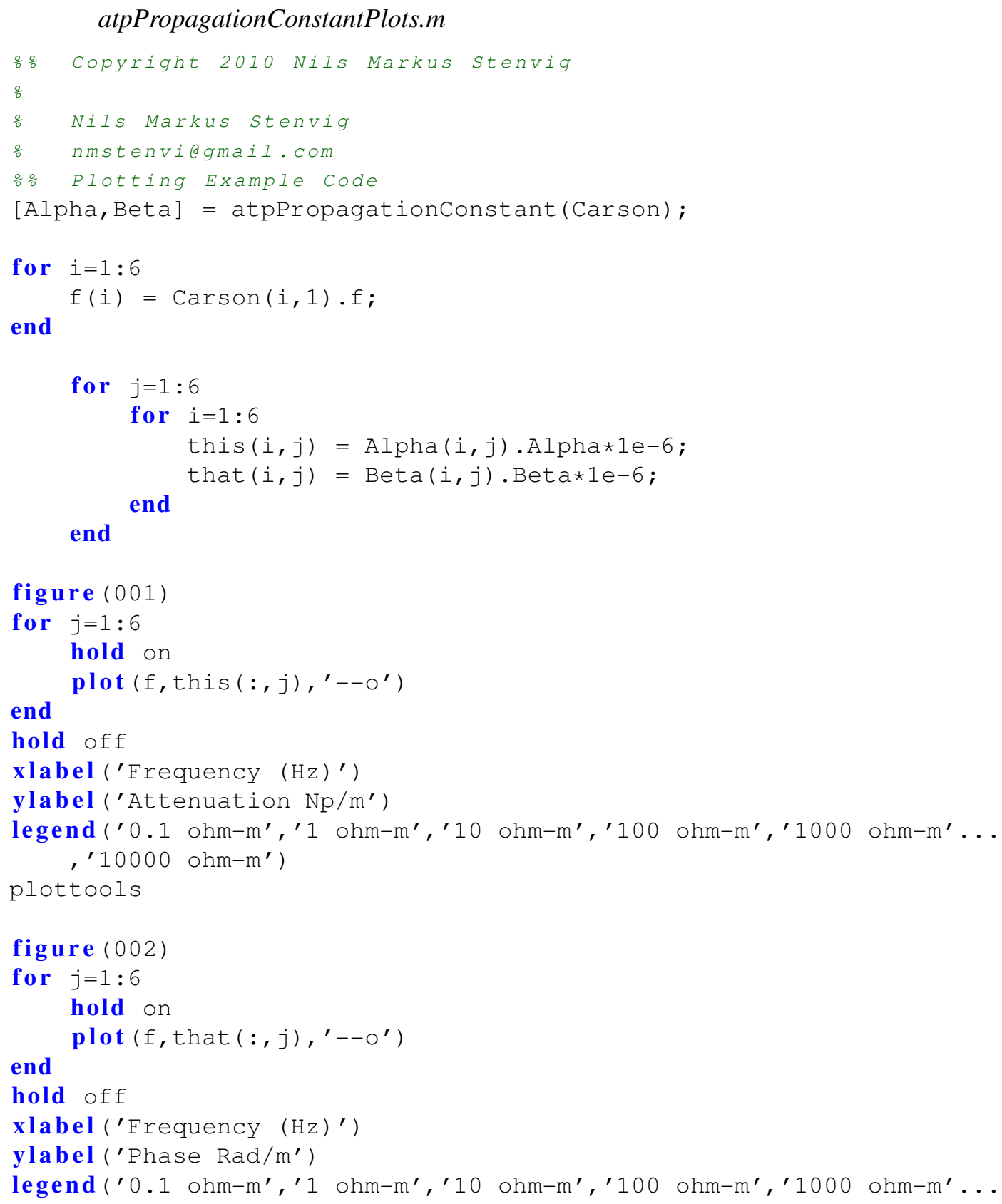


,'10000 ohm-m' )

plottools 


\section{Appendix B \\ Published Conference Paper}

B.A. Mork, N.M. Stenvig, R.M. Nelson, and B. Kirkendall, "Determination of highfrequency current distribution using emtp-based transmission line models with resulting radiated electromagnetic fields," in Power Line Communications and Its Applications (ISPLC), 2010 IEEE International Symposium on, pp. 219 Ü224, 28-31 2010.

(C) 2010 IEEE. Reprinted, with permission, from all contributing authors. Permission for reprint and republication of the following is to be obtained from IEEE. See Appendix $\mathrm{C}$ for documentation of permission for republication in this thesis. 


\section{Determination of High-Frequency Current Distribution Using EMTP-Based Transmission Line Models with Resulting Radiated Electromagnetic Fields}

\author{
Bruce A. Mork and \\ Nils M. Stenvig \\ ECE Department \\ Michigan Technological University \\ Houghton, MI USA \\ bamork@mtu.edu \\ nmstenvi@mtu.edu
}

Robert M. Nelson

Computer Engineering

University of Wisconsin - Stout

Menomonee, WI USA

r.m.nelson@ieee.org
Barry Kirkendall

Lawrence Livermore National Laboratory

Livermore, CA USA

Kirkendall1@1lnl.gov

\begin{abstract}
Application of BPL technologies to existing overhead high-voltage power lines would benefit greatly from improved simulation tools capable of predicting performance - such as the electromagnetic fields radiated from such lines. Existing EMTPbased frequency-dependent line models are attractive since their parameters are derived from physical design dimensions which are easily obtained. However, to calculate the radiated electromagnetic fields, detailed current distributions need to be determined. This paper presents a method of using EMTP line models to determine the current distribution on the lines, as well as a technique for using these current distributions to determine the radiated electromagnetic fields.
\end{abstract}

Keywords-Transmission line; frequency dependency; modeling

\section{INTRODUCTION}

When overhead high-voltage transmission lines are used as the waveguide structure for broadband communications, interference caused by the radiated emissions from those lines becomes a matter of concern [1]. While traditional Power Line Carrier (PLC) in the 25-450 kHz range [2-3], has been used for years, present Broadband over Power Line (BPL) systems operate in the $2-80 \mathrm{MHz}$ range $[1,4]$. This increase in frequency and corresponding decrease in wavelength corresponds to an increasing concern over radiated emissions.

Prediction of the radiated electromagnetic field from any antenna involves two steps: determination of the current distribution on the antenna, followed by determination of the resulting electromagnetic fields. Carrying out these steps when the 'antenna' is a realistic power system - with power lines and power system components such as transformers, capacitive banks, etc. - is a daunting task. In this paper we examine a novel two-step solution for the task.

Since this work involves two different types of modeling tools we first provide a fairly detailed background

With support from: Lawrence Livermore National Laboratory section. We examine present EMTP modeling approaches, as well as concerns that arise when using such modeling techniques at higher frequencies. We then present a unique method of applying EMTP-based transmission line models to determine the current distribution. This is followed by a description of how the radiated electromagnetic fields are determined from the current distribution. We then describe the particular test scenario used in this paper, which is followed by results and pertinent conclusions.

\section{BACKGROUND}

\section{A. EMTP Modeling Approaches}

Well known worldwide, EMTP-type software (e.g. ATP) has extensive features for modeling realistic power systems and has been successfully applied to determine PLC performance [5-6]. ATP is commonly used to determine terminal voltages and currents at characteristic power frequencies and for impulse and step response [7], although such software has not traditionally been used to determine detailed current distributions along the lines. To determine these currents using ATP we first consider the applicability and limitations of existing frequency-dependent EMTP line models which are based on physical design dimensions [8].

For higher frequencies or long lines, the two approaches that can be considered are a cascaded coupled- $\pi$ model [3Ch.11] and a distributed-parameter "long line" model. Development of presently used distributed-parameter transient transmission line models for this case are based on the "traveling wave model" or "telegrapher's model" presented in many textbooks [3-Ch.9]. The representation for a singleconductor case is shown in Fig. 1. Note that distance $(\mathrm{x})$ is measured from the receiving end toward the sending end. 


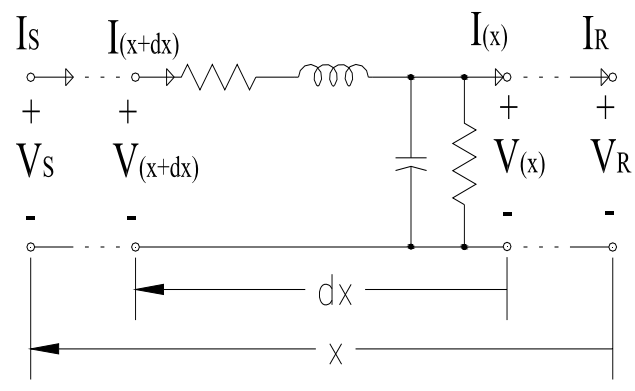

Figure 1. Telegrapher's Model

For a general multi-conductor case, the basic equations are

$$
-\frac{\partial V}{\partial x}=[Z] I \text { and }-\frac{\partial I}{\partial x}=[Y] V,
$$

where $V$ and $I$ are the vectors of node voltages and line currents at a distance $x$ from the receiving end of the multiple conductor transmission line. $\mathrm{Z}$ is the matrix of coupled series impedances of the conductors for an incremental length, and $\mathrm{Y}$ is the matrix of coupled shunt admittances for that same length. Details of solution are given in [3] and in references [9-13]. The equations from (1) can be combined to form

$$
\frac{\partial^{2} V}{\partial x^{2}}=[Z][Y] V \text { and } \frac{\partial^{2} I}{\partial x^{2}}=[Y][Z] I,
$$

where

$$
Z_{i j}=R_{i j}+L_{i j} \frac{\partial}{\partial t} \text { and } Y_{i j}=G_{i j}+C_{i j} \frac{\partial}{\partial t} .
$$

Modal transformations can be used to transform the "phase domain" equations into a set of decoupled "modal domain" equations which can simplify the mathematics for model implementation:

$$
V=\left[T_{v}\right] V_{m} \text { and } V=\left[T_{i}\right] I_{m}
$$

where $V_{m}$ and $I_{m}$ are modal voltages and currents, and $\left[\mathrm{T}_{\mathrm{v}}\right]$ and $\left[\mathrm{T}_{\mathrm{i}}\right]$ are the voltage and current transformation matrices which are also used to transform $\mathrm{Z}$ and $\mathrm{Y}$ into their decoupled modal forms $Z_{m}$ and $Y_{m}$.

$$
\begin{aligned}
& -\frac{\partial V_{m}}{\partial x}=\left[T_{V}\right]^{-1}[Z]\left[T_{i}\right] I_{m}=\left[\mathrm{Z}_{\mathrm{m}}\right] I_{m} \\
& -\frac{\partial I_{m}}{\partial x}=\left[T_{i}\right]^{-1}[Y]\left[T_{V}\right] V_{m}=\left[\mathrm{Y}_{\mathrm{m}}\right] V_{m}
\end{aligned}
$$

ATP utilizes Karrenbauer's Transformation, which is easily expanded to an arbitrary number of phases:

$$
T=\left[\begin{array}{cccc}
1 & 1 & \cdots & 1 \\
1 & (1-M) & \ddots & \vdots \\
\vdots & \ddots & \ddots & 1 \\
1 & \cdots & 1 & (1-M)
\end{array}\right],
$$

where $\mathrm{M}$ is the number of phases. The inverse transformation is of the form

$$
T^{-1}=\frac{1}{M}\left[\begin{array}{rrrr}
1 & 1 & \cdots & 1 \\
1 & -1 & 0 & 0 \\
\vdots & 0 & \ddots & 0 \\
1 & 0 & 0 & -1
\end{array}\right] .
$$

The physical representation of this for a 3-phase set of conductors is given by Figs. 2-4.

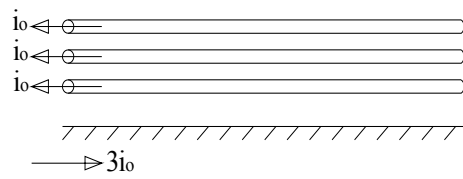

Figure 2. Mode Zero

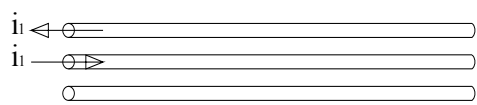

Figure 3. Mode One

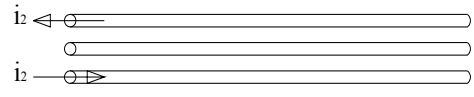

Figure 4. Mode Two

Convolution methods are used to convert the frequencydomain solution to a time-domain equivalent that can be implemented in time-domain simulation programs like EMTP. Errors in this approach are due to the fact that the solution is only valid for the frequency that the model was developed for [9-10]. Improvements have been made by applying frequencydependent weighting functions to the convolution [11-12], by developing improved frequency fitting techniques [12], and by developing the model directly in the phase domain and thus avoiding modal transformations [13]. More recent advancements include improved frequency fitting techniques [14]. In any case, it is desirable to confirm that the line model being implemented is valid within the range of frequencies to be simulated. The Foster equivalent shown in Fig. 5 is the basis for the frequency-dependent $\mathrm{Z}$.

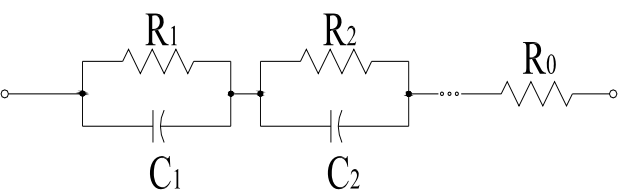

Figure 5. Foster Equivalen

Fig. 6 shows the basic representation of each end of the multi-phase Marti model [12]. Behaviors at one end manifest themselves at the other end after the appropriate propagation time delay. 


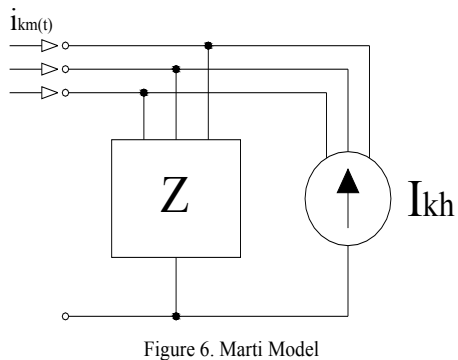

B. Electromagnetics-based Models

To accurately predict the performance of any natural phenomena (such as energy propagating on overhead transmission lines) one must pay attention to the limitations of the prediction model being used. As mentioned above, programs like EMTP are based on the "traveling wave model" or "telegrapher's model". As observed by Paul and others [1518], one of the underlying assumptions for this model is that the electromagnetic fields surrounding the transmission line structure are TEM (transverse electromagnetic) fields - i.e., that the electromagnetic fields are perpendicular to the direction of propagation (or lie in a plane transverse to direction of propagation). For the model to be strictly valid, we assume (a) the conductors are parallel to each other and to the direction of propagation, (b) they are perfect conductors (i.e., no resistance) and (c) the conductors have uniform cross section along the line axis. In addition, (d) the region surrounding the conductors is assumed homogeneous (although it can be lossy). It can also be shown (at least for twoconductor lines) that under the TEM assumption, the currents in the two conductors must be equal in magnitude and opposite in direction - i.e., that for any cross-section of the line, the total current flowing in the conductors must be zero $[15,19]$. It would appear that very few 'real life' transmission lines satisfy all of these criteria. In fact, almost all conductors have some resistive loss, lie over an imperfect ground (so they are immersed in an inhomogeneous material) and are not perfectly uniform in cross section. Although this is true, when we are examining parallel transmission lines operated at a frequency for which the cross-sectional dimensions of the line are much less than a wavelength, solution of the transmission line equations gives significant contribution to the fields and the resulting terminal voltages and currents. Such solutions are commonly referred to as 'quasi-TEM' [15] or 'quasi-static' [17] solutions. A vast body of research has been conducted evaluating when such solutions are accurate [20-23]. Olsen [17] points out that when the height of the transmission line is small compared to the wavelength in free space that the quasistatic approximation can be made, with the resulting solutions being identical to those derived by Carson [24]. Although these approximations may be valid at power frequencies, the situation changes when considering BPL frequencies when cross-sectional dimensions of the line are no longer a fraction of a wavelength.

To evaluate whether or not a given model will give accurate results one must not only ask what assumptions might be violated, but also what the results will be used for. For example, in the case of a transmission line if the desired result is to determine the terminal voltages and currents to evaluate load flows, etc., quasi-static solutions obtained from solving the transmission line equations might be perfectly acceptable. If, however, one wants to determine the electromagnetic fields radiated from the transmission lines, the error resulting from solutions based on the transmission line equations might be unacceptable. The reason is that the currents obtained from solution of the transmission line equations are truly the transmission mode (or differential line mode) currents [15-16] - i.e., currents that are flowing in opposite directions.

When the TEM assumptions are satisfied, these are the only currents that exist. When this is not the case, however, antenna mode (or common mode) currents can also exist [15-16]. These are currents that are flowing in the same direction on the lines. For most power transmission line problems, the transmission line currents are dominant, so that if one wants the terminal currents and voltages, approximate results based on transmission line theory may be perfectly adequate. It turns out, however, that in the case of radiated fields antenna mode currents tend to be very significant - even if they are much smaller in magnitude than transmission line mode currents [2526]. According to Paul [15] and Tesche [16] the reason is because the radiated fields from transmission line currents tend to subtract but those from antenna mode currents add.

To address the concern of interference potential from BPL signals propagating on power lines, researchers have turned to a number of strategies to predict the antenna mode currents (from which the resulting fields can be determined). One method is to use techniques commonly employed by those working with antennas and with other high-frequency applications of electromagnetics. A number of methods are available in the computational electromagnetics area, including the moment method, the finite element method, the finite difference method, and a host of others [16, 27-29]. Recent papers examining this issue have used a variety of techniques to analyze this problem [30-33].

One of the difficulties encountered using high-frequency methods to examine the radiated fields from practical power lines lies in modeling the multitude of components in a practical power system (i.e., transmission lines, transformers, capacitive banks, etc.). High-frequency techniques tend to work well for things like the transmission lines themselves (since they can be modeled as wires), but get cumbersome when other power system components are included in the model. Programs like EMTP-ATP, however already have lumped models for most of the power system components available. We now turn to examining how to use these models to determine the current distribution.

\section{DEVELopMent of EMTP-ATP LINE MODEL}

\section{A. Modeling Needs}

Distributed line currents and voltages are of particular interest in simulation of line performance for communications. These values are particularly important for determining the radiated fields, which are also of interest. The robust and flexible nature of EMTP-type software (e.g. ATP) makes it an ideal platform for carrying out such work. The power system 
modeling features of ATP are extensive and are used across the globe for time-domain analysis. An area that has yet to be explored, however, is in high resolution modeling of distributed currents along transmission lines. A powerful "Line \& Cable Constants" (LCC) feature of ATP is used for building transmission lines and for calculating impedance matrices. For short-line modeling, the pi approximation has been widely used. For the characteristic power frequencies there is no need to obtain highly detailed current distributions along the lines. In order to study the effects of PLC at much higher frequencies, however, the decreasing wavelengths make these highly detailed models increasingly important. The resolution of current distributions must befit the frequency being used in order to accurately calculate the radiated fields. A cascaded-pi approach is capable of meeting these needs.

\section{B. Implementation of Cascaded Models}

Transmission lines have uniformly distributed parameters while pi models are lumped parameter approximations. The pi sections modeled in ATP can be used in a cascaded approach to incrementally define the line parameters. Fig. 7 demonstrates the use of cascaded pi line sections to approximate a distributed parameter line.

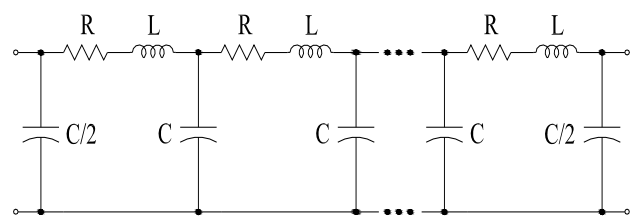

Figure 7. Cascaded Pi Representation

As mentioned before, it is necessary to have highly detailed line models when studying effects of higher frequencies and when dealing with increasingly small wavelengths. By shortening the line segments in the LCC modules of ATP, a finite number of short, cascaded pi line sections can more closely approximate a distributed parameter model. By breaking down the pi model, the line currents can be obtained for each incremental pi section. The minimum number of cascaded pi sections needed to accurately represent the line is determined by

$$
f_{\max }=\frac{N * v}{8 * \ell},
$$

where $f_{\max }$ is the maximum of the desired frequency range, $\ell$ is line length $(\mathrm{km})$, and $v$ is the propagation speed $(\mathrm{km} / \mathrm{s})$. The number of cascaded pi sections needed is thus linked to the upper limit of the desired frequency range. As the desired frequencies become very high, an obvious limitation of the cascaded approach is that a very high number of circuit elements are needed. Since the distribution of line currents is also in question, the number of simulation outputs also becomes very high. For these reasons, ATP requires a special application file designed to accommodate the higher number of circuit elements. This version, titled 'gigmingw' is readily available through the European EMTP/ATP Users Group. Because distributed line voltages and currents can be directly obtained by this method, calculation of the associated electromagnetic fields can next be achieved.

\section{DeVelopment of Radiation Model}

The Electromagnetics Interactions Generalized (EIGER) code was developed by the University of Houston, Sandia National Laboratory, and Lawrence Livermore National Laboratories. This three-dimensional, boundary element, frequency domain, code allows the computation of electric and magnetic fields from arbitrary sources built with wires, patches, and surfaces. EIGER is freely available from Sandia National Laboratory (www.sandia.gov). Ideally, radiated fields from BPL sources could be predicted entirely from EIGER However, as previously stated, transmission lines contain passive and active devices for power distribution control which cannot easily be built in EIGER; transformers being one example. Therefore, the EIGER source code was modified to accept the external ATP current distribution. Without this modification, the user would be required to accept a current distribution from a voltage or current source and approximate transmission line devices with lumped parameters; the result would be decreasing accuracy with increasing frequency.

Given a BPL current distribution calculated from ATP, the complex current is interpolated and substituted for the EIGER transmission line model current file (*.mnh). Executing the modified version of EIGER results in the ATP current distribution, EIGER model geometry, and terrain information being numerically combined into a Green's Function [34] which is then used to calculate the BPL radiated electric and magnetic fields. Using EIGER is beneficial for several reasons; the field predictions are valid into the $\mathrm{GHz}$ range, one can account for the presence of a lossy and inhomogeneous earth, and geological terrain, which might otherwise alter predicted BPL fields, can also be included in the EIGER model as dielectric bodies.

\section{TEST SCENARIO}

A test-case transmission line was identified to demonstrate the usefulness of the method for obtaining distributed line currents and radiated fields. An isolated $5 \mathrm{~km}$, 3-conductor non-transposed line was chosen for study. The flat terrain is a homogeneous ground characterized by $\varepsilon=8.0+j 1.0$ [34]. Conductor spacing is realistically defined for a standard distribution tower structure. Using the center-pole as a reference, phases A and B are left of center by 1.2192 and 0.3048 meters respectively. Phase $\mathrm{C}$ is right of center by 1.2192 meters. The conductors have a height of 9.5 meters with 0.75 meter sag and $0.03576 \Omega / \mathrm{km}$ dc resistance. The line was terminated with a small, wye-connected load of $10 \Omega$ for each phase. A current source placed at the sending end supplied a 3phase sinusoidal current as the injected signal. A frequency scan was then used to determine the current distributions for every 5 meters, with 1,000 pi sections in total, see Fig. 8 .

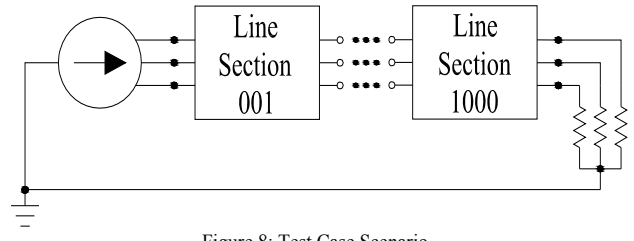

Figure 8: Test Case Scenario 


\section{RESULTS}

\section{A. Current Distribution from ATP}

Using the line description from section $\mathrm{V}$, the distributed line currents were obtained in ATP. Shown in Fig. 9 is the distributed current along one conductor as a function of line distance for a $500 \mathrm{kHz}$ injected signal. Note that the figure shows magnitudes only, and that a large source current was used for this demonstration. Though BPL systems would typically use smaller signals, the process for determining the currents and resulting fields will be the same as in this example. Additionally, while BPL frequencies are typically in the tens of $\mathrm{MHz}$, the authors wish to only describe the process in this manuscript, using a lower frequency.

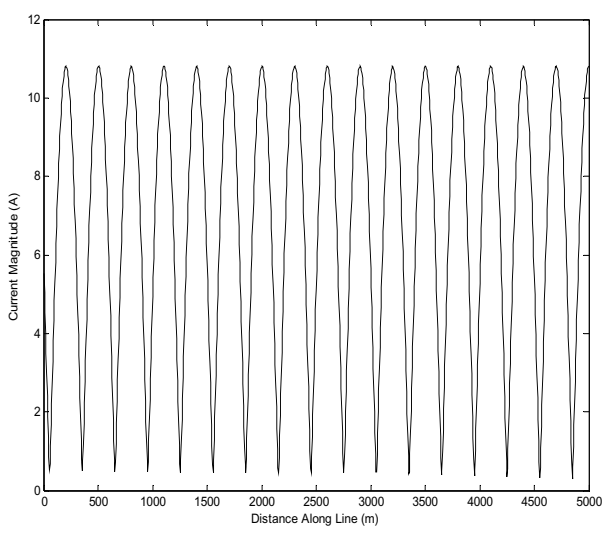

Figure 9: $500 \mathrm{kHz}$ ATP current distribution along line

B. Radiated Fields from EIGER

The $5 \mathrm{~km}$ transmission line from Section $\mathrm{V}$ is built into the EIGER model. An ASCII file of the real and imaginary transmission line current as function of line distance, and for each phase, is then created from ATP at a frequency of 500 $\mathrm{kHz}$. We do not calculate far-field patterns from EIGER, but rather a series of near field points, due to the large wavelengths at these frequencies; about 100 meters from ACSR wire with velocity of 0.33 the speed of light at $1 \mathrm{MHz}$ [35]. The fields from this test case are arbitrarily calculated at 50 meters above the transmission line in a constant altitude plane, although fields can be calculated in any volume. Fig. 10 illustrates the results for the amplitude of the vertical magnetic field (the black line represents the transmission line). Note that while Fig. 9 shows that impedance mismatches at the transmission line boundary sets up a standing wave for the current distribution with the number of nodes proportional to the frequency, the radiated fields incorporate the radiation efficiencies of the transmission lines. In essence, the current distribution and radiation efficiency are convoluted.

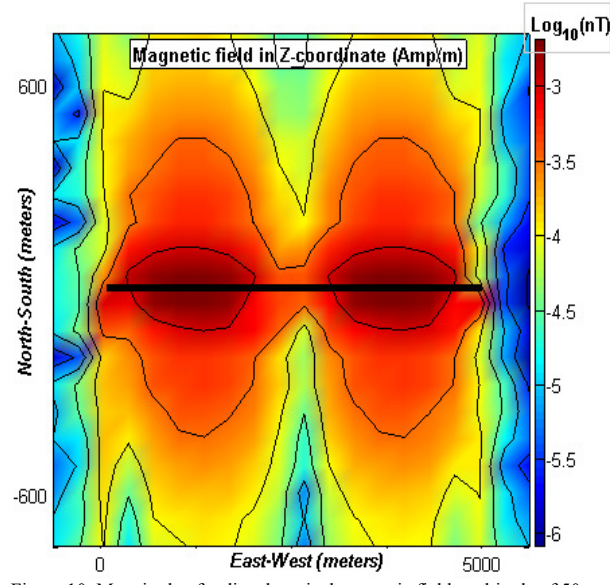

Figure 10. Magnitude of radiated vertical magnetic field at altitude of $50 \mathrm{~m}$.

\section{CONCLUSIONS AND RECOMMENDATIONS}

Radiation from BPL systems has the potential of causing interference and radiation losses from BPL systems can be significant. In order to predict both of these, EMTP-ATP is used to determine the current distribution of a transmission line. This current distribution is then overlaid onto the electromagnetic EIGER model of the physical transmission line to determine the radiated fields of a BPL system. This two-fold system is beneficial because EMTP-ATP can accurately model power electronic devices and control schemes and the components found in power systems (e.g. power transformers, instrument transformers, communication couplers, etc.) which cannot be ignored in these studies. Given this current distribution, EIGER can account for the inhomogeneity commonly found in the earth as well as using dielectric bodies to approximate terrain effects. While EIGER is valid into the microwave region $(\mathrm{GHz})$, EMTP-ATP capabilities have not been validated for such high frequencies. Future work will examine this limitation and attempt to extend EMTP-ATP into higher frequency regimes.

\section{ACKNOWLEDGMENTS}

The authors gratefully thank Ben Fasenfest at Lawrence Livermore National Laboratory for EIGER modifications and helpful discussions. Hans Kristian Høidalen at the Norwegian Institute of Science and Technology is thanked for his assistance with ATPDraw Line Constants interface to ATP.

\section{REFERENCES}

[1] L. S. Cohen, J.W. DeGraf, A. Light and F. Sabath, "The Measurement of Broadband Over Powerline Emissions," Proceedings, IEEE International Symposium on Electromagnetic Compatibility, August 8$12,2005$.

[2] H.W. Dommel, EMTP Theory Book, Microtran Power System Analysis Corporation, Vancouver, BC, May 1992.

[3] A. Greenwood, Electrical Transients in Power Systems, 2nd Edition, John Wiley \& Sons, Inc., (C1991.

[4] FCC R\&O 04-245, adopted 14 October 2004, released 28 October 2004 
[5] Modeling and Analysis of Power System Transients Using Digital Programs, IEEE Special Publication TP-133-0, IEEE Catalog No, 99TP133-0, (C1999.

[6] GE Power Management, Relaying Communications Channels Application Guide, Publication No. GET-8034, C2001.

[7] B.A. Mork, D. Ishchenko, X.Wang, A.D. Yerrabelli, R.P. Quest, C.P. Kinne, "Power Line Carrier Communications System Modeling," Proceedings, International Conference on Power System Transients, Paper No. IPST05-247, Montreal, June 19-23, 2005

[8] B.A. Mork, "Parameters for Modeling Transmission Lines and Transformers in Transient Simulations," IEEE Power Engineering Society Winter Meeting, vol. 2, pp. 716-717, 28 Jan.-1 Feb. 2001

[9] J.K. Snelson, "Propagation of Traveling Waves on Transmission Lines Frequency Dependent Parameters," IEEE Trans. PAS, Vol. 91, pp. 8591, 1973.

[10] W.S. Meyer and H.W. Dommel, "Numerical Modeling of FrequencyDependent Transmission Line Parameters in an Electromagnetic Transients Program," IEEE Trans. PAS, Vol. PAS-93, pp. 1401-1409, 1974

[11] A. Semlyen and A. Dabuleanu, "Fast and Accurate Switching Transient Calculations on Transmission Lines with Ground Return Using Recursive Convolutions," IEEE Trans. PAS, Vol. PAS-94, pp. 561-571, Recursive
1975 .

[12] J.R. Marti, "Accurate Modeling of Frequency-Dependent Transmission Lines in Electromagnetic Transient Simulations," Power Industry Computer Applications, pp. 326-334, 1981

[13] T. Noda, N. Nagaoka, A, Ametani, "Phase Domain Modeling of Frequency-Dependent Transmission Lines by Means of an ARMA Model, "IEEE Trans. Power Delivery, Vol. 11, No. 1, January 1996.

[14] A. Morched, B. Gustavsen, and M. Tartibi, "A Universal Model for Accurate Calculation of Electromagnetic Transients on Overhead Lines and Underground Cables", IEEE Trans. Power Delivery, vol. 14, no. 3 pp. 1032-1038, July 1999 .

[15] C. R. Paul, Analysis of Multiconductor Transmission Lines, $2^{\text {nd }}$ Edition, Wiley-IEEE Press, C2007.

[16] F. M. Tesche, M. Ianoz, T. Karlsson, EMC Analysis Methods and Computational Models, Wiley-Interscience, (C)1996.

[17] R. G. Olsen, "Power-Line Electromagnetics," IEEE Antennas and Propagation Magazine, Vol. 36, No. 6, pp. 7-16, December 1994

[18] R. F. Harrington, Time-Harmonic Electromagnetic Fields, Mc-Graw Hill, (C1961

[19] C. W. Harrison, "Derivation of the transmission-line equations from linear antenna theory," IEEE Antennas and Propagation Magazine, Vol. 36, No. 6, pp. 33-34, December 1994.
[20] J. R. Wait, "Theory of wave propagation along a thin wire parallel to an interface," Radio Science, Vol. 7, pp. 675-679, June 1972.

[21] E. F. Kuester, D. C. Chang, and R. G. Olsen, "Modal Theory of Long Horizontal Wire Structures Above the Earth, 1, Excitation,"Radio Science, Vol. 13, pp, 605-613, July-August 1978.

[22] A .F. Dos Santos and J. P. Figanier, "The Method of Series Expansion in the Frequency Domain Applied to Multiconductor Transmission Lines," IEEE Trans. on Microwave Theory and Tech., Vol. MTT-23, pp. 753 756, September 1975.

[23] D. C. Chang and R. G. Olsen, "Excitation of an Infinite Horizontal Wire Above a Dissipative Earth," Radio Science, Vol. 10, pp. 823-831, August- September 1975

[24] J. R. Carson, Wave Propagation in Overhead Wires with a Ground Return, Bell Sys. Tech. J., Vol. 5, pp. 539-554, October 1926.

[25] C. R. Paul and D. R. Bush, "Radiated Emissions from Common-Mode Currents," IEEE International Symposium on Electromagnetic Compatibility, Atlanta, GA, September 1987

[26] C. R. Paul and D. R. Bush, "A Comparison of the Contribution of Common-Mode and Differential-Mode Currents in Radiated Emissions," IEEE Trans. on Electromagnetic Compatibility, Vol. EMC-31, pp. 189$193,1989$.

[27] R.F. Harrington, Field Computation by Moment Methods, IEEE Press, (C) 1993.

[28] A.F. Peterson, S. L. Ray and R. Mittra, Computational Method for Electromagnetics, IEEE Press, (C1998

29] M.N.O. Sadiku, Numerical Techniques in Electromagnetics ,CRC Press, (C)2001.

[30] M. Karduri, M. D. Cox and N. J. Champagne, "Near-Field Coupling Between Broadband Over Power Line (BPL) and High-Frequency Communication Systems," IEEE Trans. on Power Delivery Vol. 21, No. 4, pp. 1885-1891, October 2006.

[31] S. Cerwin, "Broadband Over Powerline (BPL) Signal Leakage Analysis Using NEC Modeling With Experimental Verification", [Online] Available: http://www2.arrl.org/tis/info/HTML/plc/files/Cerwin.pdf.

[32] M.D'Amore and M. S. Sarto, "Electromagnetic Field Radiated from Broadband Signal Transmission on Power Line Carrier Channels," IEEE Trans. on Power Delivery, Vol. 12, No. 2, pp. 624-631, April 1997.

[33] F. M. Tesche, b. A. Renz, R. M Hayes, R. G. Olsen, "Develoment and Use of a Multiconductor Line Model for PLC Assessments," International Symposium on Electromagnetic Compatibility, Zurich, pp. 99-104, February 2003

[34] C. Balanis, Advanced Engineering Electromagnetics, Wiley, C1989 


\title{
Appendix C
}

\section{Documentation of IEEE Republication Permission}

\author{
Comments/Response to Case ID: 006 E2368 \\ ReplyTo: Copyrights@ieee.org \\ From: Jacqueline Hansson Date: 02/25/2011 \\ Subject: Re: Publication Send To: Nils Stenvig \\ Permissions <nmstenvi@gmail.com> \\ Dear Nils Stenvig : \\ This is in response to your letter below in which you have requested \\ permission to reprint, as part of an appendix to your report/publication, \\ the described IEEE copyrighted material. We are happy to grant you this \\ permission. \\ Our only requirements are that you obtain the approval of the senior author \\ and his company (unless you are the senior author) before you consider our \\ permission final, and that the following IEEE copyright/credit notice \\ appears in a prominent place in the appendix, with the appropriate details \\ filled in: \\ (c) 2010 IEEE. Reprinted, with permission, from (complete publication \\ information). \\ Sincerely yours, \\ Jacqueline Hansson \\ (c) (ㄷ) (ㄷ) (c) (c) (c) (c) (c) (c) (c) (c) (c) (c) (c) (c) (c) (c) (c) \\ IEEE Intellectual Property Rights Office \\ 445 Hoes Lane \\ Piscataway, NJ 08855-1331 USA \\ +17325623966 (phone) \\ +17325621746 (fax) \\ IEEE-- Fost ering technological innovation \\ and excellence for the benefit of humanity.

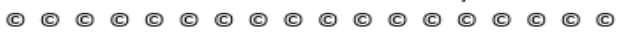


To whom it may concern,

I am seeking republication rights of an IEEE conference paper. I am an author of the publication of interest, and I intend to use the paper (in its entirety) as an attachment to my Masters thesis. The paper details follow.

Authors: Mork, B.A. ; Stenvig, N.M. ; Nelson, R.M. ; Kirkendall, B. ;

Paper Title: Determination of high-frequency current distribution using

EMTP-based transmission line models with resulting radiated electromagnetic

fields.

Pub. Title: Power Line Communications and Its Applications (ISPLC), 2010

IEEE International Symposium on

E-ISBN : 978-1-4244-5010-7

Print ISBN: 978-1-4244-5009-1

I would appreciate to hear back as soon as possible.

Best regards,

Nils Markus Stenvig

nmstenvi@mtu.edu

nmstenvi@gmail.com

Michigan Technological University

Department of Electric Power Engineering

1400 Townsend Drive, EERC SB-26

Houghton, MI 49931 


\section{Appendix D}

\section{Notes on Continuation of Research Work}

The conclusion of this thesis includes recommendations for future work in continuation of what has been presented. It is the recommendation of the author that this thesis be used as a tool for learning and understanding the necessary background for working in the realm of high-frequency wave propagation and radiated fields. The first three chapters would provide an introduction to transmission line theory and BPL, while the latter chapters describe the modeling and implementation details of the investigation methods. Underlying these issues, however, is a need for an understanding and familiarity with concepts of electromagnetics, circuit theory, and the use of certain software programs. At a minimum, the following items outline the necessary background to continue the research.

- Must be well versed in power system analysis concepts. Undergraduate-level power systems and electromagnetics courses would be a minimum requirement.

- Understanding of advanced topics in power systems would be more beneficial. Particularly in transient analysis, transmission line theory, linkage between time-domain and frequency domain modeling, and electromagnetics.

- This research would require sufficient understanding of the use of EMTP/ATP as well as the formulas and implementation details of many features. It would be very difficult to continue this research withouth being well versed with ATP.

- At least one person involved with further research should understand the EIGER program and be able to use it (EIGER is introduced in Section 3.1.3).

- A significant amount of programming will be required in advancement of this research. As such, programming skills would be necessary (eg. - Matlab, Python, ATP-Models).

- This thesis can be used to understand many advanced concepts, but many things cannot be understood without the background mentioned above.

- In addition, an understanding of the vector fitting program (Section 5.3.2) would be beneficial. The vector fitting package includes a user's manual to help in this regard. 